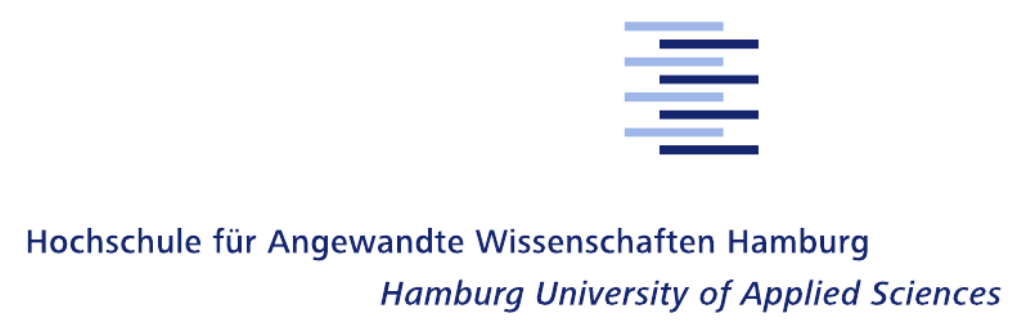

Hamburg University of Applied Sciences

\title{
Master Thesis
}

\author{
Malte Nogalski
}

\section{Acoustic Redirected Walking with Auditory Cues by Means of Wave Field Synthesis}

Fakultät Technik und Informatik

Studiendepartment Informatik
Faculty of Engineering and Computer Science

Department of Computer Science 


\section{Malte Nogalski \\ Acoustic Redirected Walking with Auditory Cues by Means of Wave Field Synthesis}

Master Thesis submitted in terms of

the degree course Computer Science at the Department of Computer Science

Faculty of Engineering and Computer Sciences

Hamburg University of Applied Sciences

Supervisor: Prof. Dr. Wolfgang Fohl

Co-Supervisor: Prof. Dr. Kai von Luck

Submitted on: 27th August 2015 


\title{
Malte Nogalski
}

\section{Title of the paper}

Acoustic Redirected Walking with Auditory Cues by Means of Wave Field Synthesis

\section{Keywords}

Acoustic Redirected Walking (RDW), Wave Field Synthesis (WFS), Immersive Virtual Environment (IVE), Virtual Locomotion User Interface, Human-Computer Interaction (HCI)

\begin{abstract}
I present an experiment to identify detection thresholds for acoustic redirected walking by means of a wave field synthesis system. 39 blindfolded test subjects performed 2777 constant stimulus trials with rotation and curvature gains of different severity. The detection thresholds were greatly dependent on the knowledge the subjects had of the experiment. The most representative test group noticed an up-scaling rotation of $5 \%$ and a down-scaling rotation of $37.5 \%$, and a circular path with a radius of 5.71 meters $25 \%$ of the time. Almost no signs of simulator sickness could be observed with the simulator sickness questionnaire by Kennedy et. al.
\end{abstract}

\section{Malte Nogalski}

\section{Thema der Arbeit}

Akustisches Redirected Walking mit Orientierungsreizen durch eine Wellenfeldsyntheseanlage

\section{Stichworte}

Akustisches Redirected Walking (RDW), Umgeleitetes Gehen, Wellenfeldsynthese (WFS), Immersive Virtuelle Umgebung (IVE), Virtuelle Fortbewegungsbenutzerschnittstelle, MenschComputer-Interaktion (MCI)

\section{Kurzzusammenfassung}

Ich präsentiere ein Experiment zur Identifikation von Erkennungsschwellwerten akustischer Redirected Walking Anwendungen mittels einer Wellenfeldsynthese-Anlage. 39 Probanden mit verbundenen Augen führten 2777 einzelne Tests durch, in denen ihre Rotationen vergrößert oder verkleinert wurden und ihre Pfade gebeugt wurden. Die relevanteste der vier Testgruppe bemerkte es mit einer Wahrscheinlichkeit von $25 \%$, wenn ihre Rotationen um mehr als $5 \%$ vergrößert oder mehr als 37,5\% verkleinert wurden oder ihr gerader Weg zu einem Kreis mit einem Radius von weniger als 5,71 metern gebogen wurde. 


\section{Contents}

$\begin{array}{lr}\text { 1. Introduction } & 1\end{array}$

1.1. Motivation ........................ 2

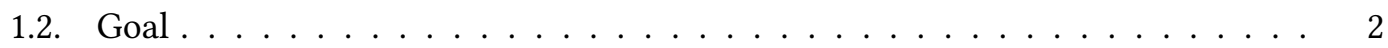

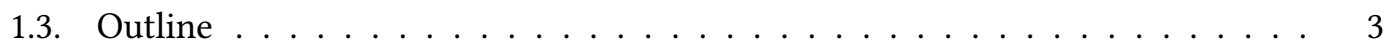

2. Related Work $\quad 5$

2.1. General Redirected Walking (RDW) . . . . . . . . . . . . . . . . . 5

2.2. Algorithms to Dynamically Apply Gains . . . . . . . . . . . . . . . . . . 8

2.3. The Human Locomotion Triple (HLT) . . . . . . . . . . . . . . . . . . . . . . 9

2.4. Gains to Manipulate the Users' Movements . . . . . . . . . . . . . . . . . . . 10

2.5. Experiments for Detecting Thresholds . . . . . . . . . . . . . . . . . . 13

2.6. Physical Obstacles . . . . . . . . . . . . . . . . . . . . . . . . . 14

2.7. Velocity Dependency of Redirected Walking Techniques . . . . . . . . . . . 15

2.8. Reorientation Technique (ROT)s and Distractors . . . . . . . . . . . . . . . . 16

2.9. Non-Visual Redirected Walking by Acoustic Stimuli . . . . . . . . . . . . . . 17

2.10. Cyber Sickness . . . . . . . . . . . . . . . . . . . . . 20

3. Wave Field Synthesis $\quad 23$

3.1. Motivation . . . . . . . . . . . . . . . . . . 23

3.2. The Principle . . . . . . . . . . . . . . . . . . . . 24

3.3. Properties of Virtual Sound Sources . . . . . . . . . . . . . . . . . . . . . . . . . . . . . . . . . 25

3.4. Technology . . . . . . . . . . . . . . . . . 25

3.5. Operating Options . . . . . . . . . . . . . . . . . . . 27

3.5.1. Open Sound Control . . . . . . . . . . . . . . 27

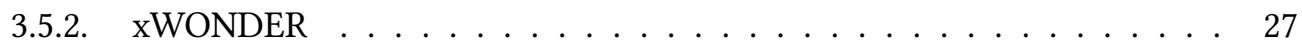

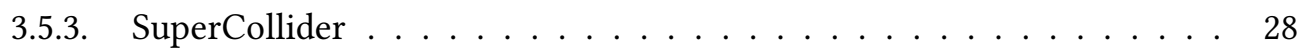

4. Tracking Methods and Systems $\quad 30$

4.1. Tracking Systems in General . . . . . . . . . . . . . . . . . . . . . . 30

4.2. Differences Between Tracking Methods and Systems . . . . . . . . . . . . . . 30

4.3. Requirements of the Redirected Walking Component for the Tracking System 31

5. The Laboratory $\quad 32$

5.1. Motion Tracker-Wave Field Synthesis-Connector (MoWeC) . . . . . . . . . . . 32

5.2. Redirected Walking Component . . . . . . . . . . . . . . . . . . . 35 
6. Choice of Gains to be Tested

7. Experiment Design 38

7.1. Requirements . . . . . . . . . . . . . . . . . . . 38

7.1.1. Ambient Noise . . . . . . . . . . . . . . . . . . . . . . . 38

7.1.2. Sounds to Turn . . . . . . . . . . . . . . . . . . . . . . . . 39

7.1.3. Sounds to Walk . . . . . . . . . . . . . . . . . . . . . . . . . 39

7.2. Automated Test Sequence . . . . . . . . . . . . . . . . . . . . . . . . 40

7.2.1. Conditions . . . . . . . . . . . . . . . . . . . . . . 40

7.2.2. Path Prediction . . . . . . . . . . . . . . . . . . . . . 40

7.2.3. Automation . . . . . . . . . . . . . . . . . 42

7.3. Gain Values . . . . . . . . . . . . . . . . . . . . . . . . 43

7.4. Test Groups . . . . . . . . . . . . . . . . . . . . . . . 44

8. Results 46

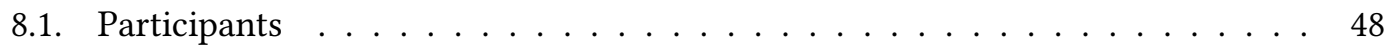

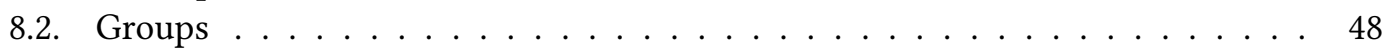

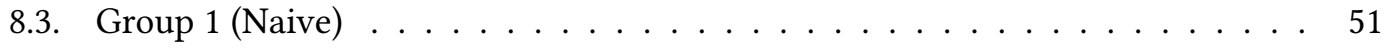

8.4. Group 2 (Aware) . . . . . . . . . . . . . . . . . . . . . 52

8.5. Group 3 (Expert) . . . . . . . . . . . . . . . . . . . 53

8.6. Group $4(2 \mathrm{AFC}) \ldots \ldots \ldots \ldots \ldots \ldots$

8.7. Comparison of Detection Thresholds _ . . . . . . . . . . . . . . . 60

8.7.1. Rotation Gain . . . . . . . . . . . . . . . . . . . . . . . . . . . . . 60

8.7.2. Curvature Gain . . . . . . . . . . . . . . . . . . . . . . . . . . . 60

8.8. Exemplary Paths . . . . . . . . . . . . . . . . . . . 61

8.9. Detection Rates by Test Number . . . . . . . . . . . . . . . . . . . . . 66

8.10. Velocity Dependency . . . . . . . . . . . . . . . . . . . . 67

8.11. Cyber Sickness . . . . . . . . . . . . . . . . . . . . . . . . . . 68

$\begin{array}{ll}\text { 9. Conclusions } & 70\end{array}$

10. Summary and Future Work

10.1. Summary . . . . . . . . . . . . . . . . . . . . . 74

10.2. Future Work . . . . . . . . . . . . . . . . . . . 75

A. Functions $\quad \mathbf{7 6}$

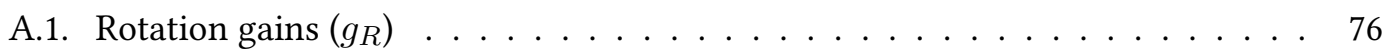

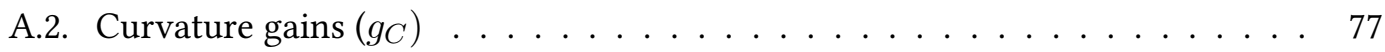

$\begin{array}{ll}\text { B. Test subjects } & 78\end{array}$

$\begin{array}{lc}\text { Glossary } & 83\end{array}$

$\begin{array}{lr}\text { Acronyms } & \mathbf{8 8}\end{array}$ 


\section{Introduction}

With projects like the Google Glasses ${ }^{1}$, the Oculus Rift ${ }^{2}$, the Microsoft HoloLens ${ }^{3}$, and powerful rendering hardware, virtual realities (VRs) ${ }^{4}$ and mixed realities (MRs) currently experience kind of a renaissance and become more and more realistic. While all areas of human computer interfaces (HCIs) aspire to be easy and intuitive to use, VRs and MRs provide special challenges due to their immersiveness. Especially free gestures come into focus in that context. The most natural and intuitive way, to navigate a human-like avatar through an immersive virtual environment (IVE), is by physically walking (Ruddle and Lessels, 2009), and many applications of 3D games, urban planing, tourism (Beimler et al., 2013) and training scenarios would benefit from an interface like that, but for obvious reasons that option is mostly not available. The tracking and evaluation of the physical movements is well within the possibility of many scenarios, but what remains is the limited physical space, as the main hurdle (Walker, 2013). Some approaches tackle that hurdle by reducing the physical movements to mimicking a walk-like gesture (Usoh et al., 1999; Razzaque et al., 2002), and another popular approach is letting the users walk on treadmills (Brooks Jr et al., 1992; Darken et al., 1997; Hollerbach et al., 2000; Bouguila and Sato, 2002; Souman et al., 2011; Frissen et al., 2013; Sinitski et al., 2015) or foot pedals (Vu et al., 2015), but real walking remains the best choice regarding naturalism.

The redirected walking $(R D W)$ approach tries to retain all the naturalism of real walking, by exploiting the limitations of human perceptual mechanisms for sensing position, orientation and movement (Razzaque et al., 2001), and at the same time allow the exploration of an IVE with potentially infinite extend (Razzaque et al., 2001). Most people, can't walk on a straight line solely by relying on their proprioception ${ }^{5}$ (Souman et al., 2009). A certain degree of inaccuracy has to be corrected, by in general mostly visual, but also extraretinal cues.

\footnotetext{
${ }^{1}$ http://www.google.com/glass/start/

${ }^{2}$ https://www.oculus.com/en-us/

${ }^{3}$ https://www.microsoft.com/microsoft-hololens/en-us/

${ }^{4}$ Italic terms can be found in the glossary

${ }^{5}$ Proprioception: Perception governed by proprioceptors, as awareness of the position of one's body. (proprioception, 2015)
} 
"Extraretinal cues come from the vestibular system, proprioception, our cognitive model of the world, or from an efference copy of the motor commands that move the respective body parts." (Steinicke et al., 2008a)

These corrections, that are part of every step we take, are executed subconsciously. $R D W$ manipulates the visual and/or acoustic cues selectively, and the manipulated persons ideally subconsciously compensate these manipulations by steering into the intended direction without it ever reaching their consciousnesses.

The ultimate goal for a redirected walking application would be to create an IVE, which is somewhat comparable with the star trek holodeck (Steinicke et al., 2008e; Wikipedia, 2014), where users can roam freely and unlimited within an highly sophisticated and extensive IVE.

\subsection{Motivation}

Redirected walking $(R D W)$ offers an approach to limit the required physical space, while maintaining all the naturalism of real walking, and had much attention within the last decade. However, most of the research focused very strongly on navigation by visual cues and the acoustic aspect had been neglected pretty much or did not find any appreciation at all. A lot of researchers however agree, that a coherent acoustic component would at least support the visual representation and help the $R D W$ techniques to archive their goal (Field and Vamplew, 2004). Other scenarios might not allow a visual representation of the IVE at all. Anything for blind people would have to manage without a visual representation, but also in special situations in games or training simulations, a user might have to switch to other means of navigation, but visual cues. While there are quite a few working examples of visual $R D W$, "vision usually dominates proprioceptive, i. e., vestibular and kinesthetic, sensation when the two disagree" (Gibson, 1933; Dichgans and Brandt, 1978; Berthoz, 2002). So the question, how well a redirected walking application would work without the dominant visual sense, remains. For acoustic orientation a good spatial representation of the IVE is most crucial and wave field synthesis (WFS) is probably the most sophisticated spatial sound reproduction system today. Therefore a detailed investigation into its potential for redirected walking applications will help advance the research in this field.

\subsection{Goal}

This work shall essentially contribute to the identification of detection thresholds for manipulations of different severity for acoustic RDW applications and in the end also for audiovisual 
redirected walking applications. It shall help to illuminate the acoustic aspect of redirected walking applications and also identify characteristics, that are particular to acoustic $R D W$ in comparison to visual $R D W$. Would people be less sensitive to acoustic $R D W$ manipulations, because they are less trained in acoustic orientation and therefore more likely to be consent, or would the absence of the dominant visual sense stimulate them to rely more on their vestibular system and proprioception, and would therefore a more equal balance between the senses result in a higher detection probability?

How will knowledge of the system affect the detection probability of users of an acoustic $R D W$ application? The knowledge, whether or not a user is expecting to be manipulated by a dynamic virtual environment (VE), will probably change the awareness towards addressed occurrences. Different test groups shall give insight into the effect, merely the introduction to the experiment will have on the results and therefore enable developers of an acoustic $R D W$ application to adjust algorithms accordingly and individually. Furthermore it shall emphasize on the importance to factor in the anticipation of test subjects towards the system for further experiments.

Last but not least the question shall be answered, whether or how severe symptoms of cyber sickness are to be expected for users of an acoustic $R D W$ application during and after a medium period of time of blind navigation within an unfamiliar acoustic IVE, which behaves unnatural and unpredictable.

Due to an experimental design related to that of the most relevant related work, the results shall be comparable as best as possible.

\subsection{Outline}

Subsequent to this introduction this thesis will start with giving an insight into the most relevant and recent related work. Starting with an introduction into general $R D W$, the chapter will illuminate $R D W$ algorithms and different $R D W$ techniques. It will then focus on some of the more important special cases like physical objects for the $V E$, emergency procedures, the acoustic aspect in previous $R D W$ experiments and cyber sickness.

An introduction to WFS, its principle, advantages, applications, technology and operation options will be given, as well as some information about different tracking methods and systems for spatial person/object tracking will be given, before focusing on the requirements of a tracking system for an acoustic RDW application. The laboratory of the University of Applied Sciences Hamburg (UAS Hamburg) will be introduced briefly and some implementation details about the software written to conduct the experiment will be given. 
The process to select the relevant $R D W$ techniques for the experiment at hand will be illustrated and the experiment design will be explained from the requirements over the test sequence of the experiment up to the building of the test groups.

The presentation of the results will start with an overview of the 39 test subjects, and then illustrate the merged resulting thresholds of all test groups after which the identified thresholds of the four different test groups are presented separately. In addition to the thresholds some exemplary paths will be presented to give insight into different behaviours of test subjects during the trials. The presentation of the results will end with an evaluation of detection rate by time within the $V E$, velocity dependency and possible effects of cyber sickness.

After the results have been presented, they will be discussed, examined in contrast to each other, and some characteristics that stick out particular due to the acoustic aspect of the experiment will be illuminated.

Finally a summary and some ideas for future work will be given. 


\section{Related Work}

This chapter will give an introduction to the basic concepts and algorithms of $R D W$. Various approaches to apply gains to manipulate users' movements are reviewed, and the reported thresholds for the identification of these manipulations are summarized for both visually and non-visually guided $R D W$.

\subsection{General Redirected Walking (RDW)}

$R D W$ exploits the limitations of human perceptual mechanisms for sensing position, orientation and movement (Razzaque et al., 2001; Walker, 2013; Engel et al., 2008; Souman et al., 2009). Due to these limitations, people can and will even walk in circles while attempting to walk in a straight line, when the environment is too sparse to give enough distinctive cues for reorientation Souman et al. (2009). A certain degree of inaccuracy has to be corrected by in general mostly visual, but also extraretinal cues.

"Extraretinal cues come from the vestibular system, proprioception, our cognitive model of the world, or from an efference copy of the motor commands that move the respective body parts." (Steinicke et al., 2008a)

These corrections, that are part of every step we take, are executed subconsciously. $R D W$ manipulates these

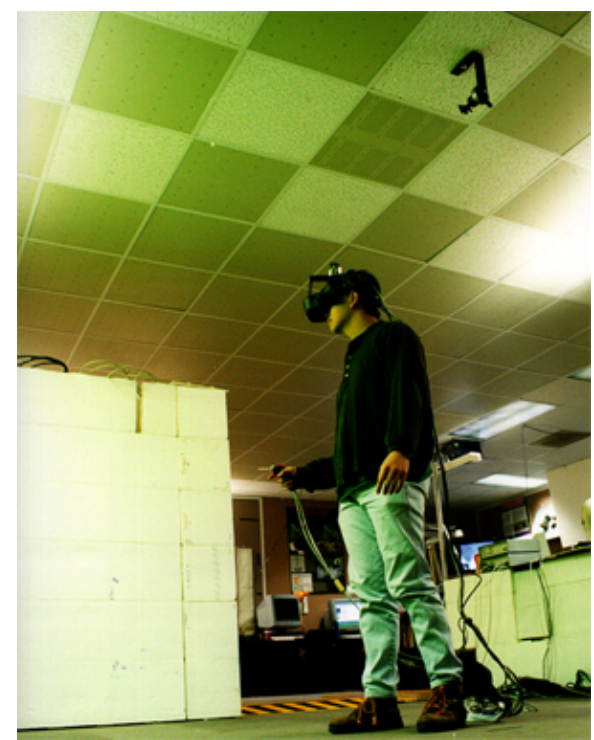

Figure 2.2.: A user wearing the head mounted display (HMD) and the wide area tracker during the experiments for (Razzaque et al., 2001).

additional cues selectively, and the manipulated persons, who perceive these external-motions (the objects / the world around them move) as self-motion (the person moves), subconsciously 


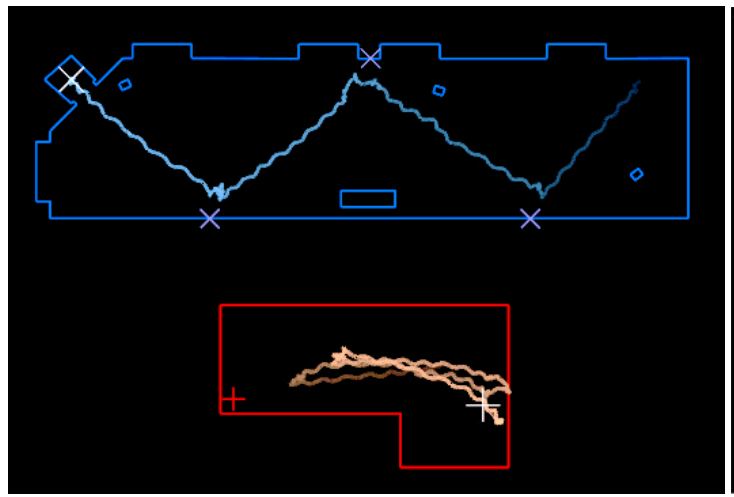

(a) Overhead views of the path taken by the user in the virtual environment (above in blue) and the laboratory (below in red). Note how the user walked in a zigzag pattern through the $V E$ while walking back and forth within the tracker space. The tracker space and $V E$ are drawn to scale. Figure taken from (Razzaque et al., 2001)

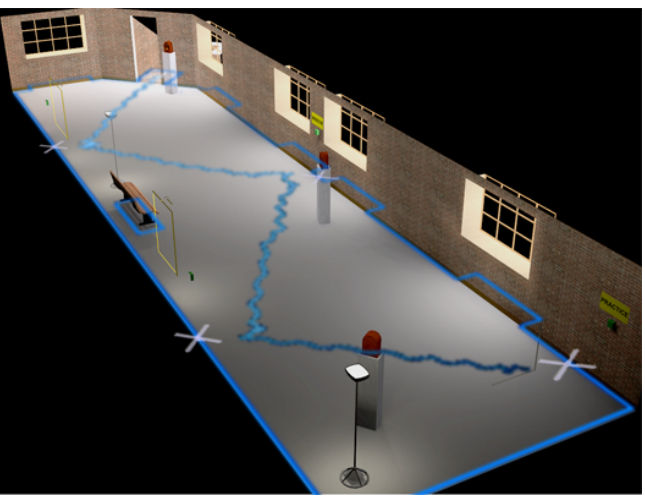

b) The user's path superimposed onto the VE. (Razzaque et al., 2001)

Figure 2.1.: Two pictures of the fire drill experiment in (Razzaque et al., 2001). Crosses denote static waypoints.

compensate these manipulations by steering into the intended direction without it ever reaching their consciousnesses.

Humans rely primarily on vestibular, visual and auditory cues for balance and orientation (Dichgans and Brandt, 1978). These cues are used to perceive the real world as stable as we move, and to distinguish between self-motion and external-motion (Wallach, 1987; Bridgeman et al., 1994; Wertheim, 1994). A consistency of multiple kinds of those cues may increase the chance that external-motion may be perceived as self-motion (Lackner, 1977).

The ultimate goal for a redirected walking application would be to create an IVE, which is somewhat comparable with the star trek holodeck (Steinicke et al., 2008e; Wikipedia, 2014), where users can roam freely and unlimited within an highly sophisticated and extensive (potentially infinite) IVE.

According to Razzaque et al., Michael Moshell and Dan Mapes made their first attempts to manipulate users of VEs into unknowingly walking along arcs while thinking, that they were walking on a straight line in 1994 at the University of Central Florida (Razzaque et al., 2001). They used means of visual stimulation for their redirection technique, but could not elude the problems of what they identified as simulator sickness (Kennedy et al., 1993) and the limitations of $V E$ systems, in particular the tracking systems. 
In 2001 Razzaque et al. assume, that $R D W$ might now be possible with the recent development in VEs as well as accurate, low latency, wide-area tracking systems and therefore address the problem once again (Razzaque et al., 2001).

Their technique rotates the $V E$ around the user in such a way, that the user is made to always walk towards the farthest wall of the tracking area. In theory and with a tracking area large enough, according to Razzaque et al., it should be possible to present a $V E$ of infinite extent. However, with a decrease of tracking area, eventually more rotation has to be applied to the $V E$, to keep the user within the physical perimeter of the tracking area, and each increase of applied rotation also increases the chance of the user detecting the manipulation. The thresholds for applied rotational distortion is therefore a tradeoff between a lower detection probability and less physical space requirement. For limited tracking areas, Razzaque et al. propose to force the user to look around/rotate around their own axis at strategically placed waypoints, as illustrated in figure 2.1b. During these rotations a good amount of rotational distortion can be hidden within the natural perceived rotation of the senses for orientation and balance of the user. While these waypoints impose a mayor constraint, they believed that a lot of applications (such as their experiment, which will be described here shortly) naturally offer reasonable waypoints.

Razzaque et al. even claim, that "Even while standing still, the user unknowingly rotates her head and torso with the virtual scene" and assume, that the explanation lies within the user's own balance system in regard to Dichgans and Brandt (1978).

Within their experiment, which is often referred to as the first working case of $R D W$ (Neth et al., 2012; Hodgson and Bachmann, 2013), Razzaque et al. generated a fire drill within a virtual hallway as illustrated in figure 2.1b. For this experiment the users wore a head mounted display with stereo headphones to present the visuals and spatialized audio (see figure 2.2). The task was to calmly move to four different positions along the hallway in figure $2.1 \mathrm{~b}$, and push some virtual buttons. After each button, the users were to first turn towards the next target, and then move straight towards that target without wandering around. During these turns towards the next target, a rotational scaling was applied to the representation of the $V E$, to point the user towards the direction she came from, while making her see, hear and believe, that she turned towards a position further down the hallway. The blue box in figure 2.1a shows the path, the user has taken within the VE. The red box below shows the path she took within the tracking area at the same time. Small misalignments after the turns were corrected by further applying small rotational distortions, while the user was walking towards the next waypoint. This explains the arcs in figure 2.1a and also serves as an example of $R D W$ while actually walking and not rotating on the spot. So while actually walking back and forth 
in a rather small physical room, the user had the impression of advancing along a significantly lager area. This experiment should also work for a virtual hallway of infinite length. The subjects of the experiment did not see the laboratory before starting the experiment, were unfamiliar with the building and all of them were surprised about the small size of the lab after removing the headset.

For situations during the algorithm development phase in which a user nonetheless came upon a boundary of the tracking area, Razzaque et al. used a virtual instructor to tell the user to stop walking and look to the left and right. The turning of their heads gave the algorithm the opportunity to apply additional rotational distortions to aim the users away from the wall back into the tracking area without them noticing the change of orientation of up to more then 90 degrees. More about these reorientation techniques (ROTs) will follow in section 2.8 (Reorientation Technique (ROT)s and Distractors). In (Lubos et al., 2014) Lubos et al. introduce an idea on how to use $R D W$ in especially small laboratories. Users, when walking virtually straight, would be redirected to be walking on a path with the maximum possible radius within the physical workspace i. e. on the outer boundaries of the largest possible circle within the laboratory. The dynamic opacity of a semi-transparent half-capsule warns the user, when approaching the boundaries of the physical laboratory or tracking area, and whenever the user approaches an object of interest, she is redirected towards the center of the circle before she reaches it. A large area around the center of the circle is considered the save area, in which users can move without having to be redirected. Only when approaching the outer region of the circle, the $R D W$ algorithm is calculating the minimum angle necessary, to guide them on the outer region around the inner circle.

Approaches that merely mimic a walk-like gesture, but keep the user in place (Razzaque et al., 2002; Usoh et al., 1999) elude the problem of limited tracking areas altogether and are also still pursued (Nilsson et al., 2014a,b), but can not keep up with the naturalness of real walking (Chance et al., 1998; Usoh et al., 1999; Lee and Hidler, 2008)

In 2005 Razzaque published his $\mathrm{PhD}$ on $R D W$ (Razzaque, 2005).

\subsection{Algorithms to Dynamically Apply Gains}

The algorithm used in (Razzaque et al., 2001) is shown in figure 2.3, and was customized for the fire drill experiment of Razzaque et al.. As mentioned before, in (Razzaque et al., 2001) users even compensated for small amounts of rotational distortion to the VE while standing still. This is the baseline constant rotation and the first of three basic factors to the resulting rotational distortion. The other two are a scaling to the users physical rotation and a rotation 
proportional to the users linear velocity (i. e. walking speed), but only the maximum of those three would find consideration and be scaled by the sine of the angle between the next virtual target and the next physical target. Finally the resulting distortion rate was limited by a fixed threshold, which was determined as being the threshold for imperceptible rotational distortion by previous tests.

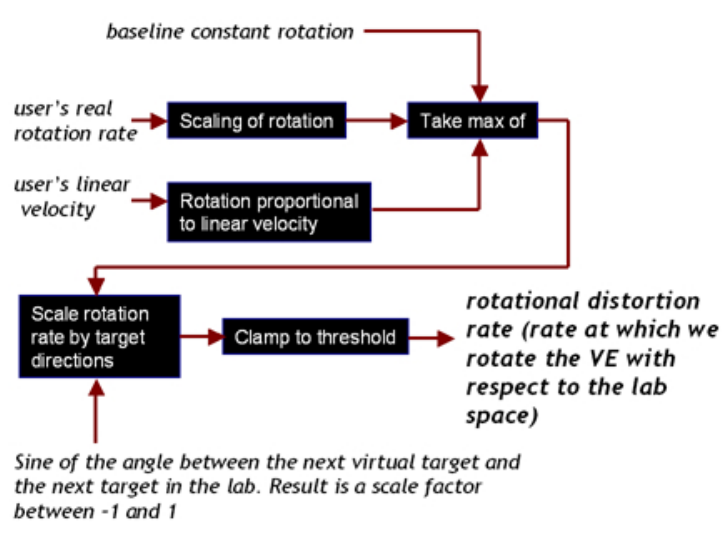

Figure 2.3.: The algorithm for computing the rotational distortion rate. Figure taken form (Razzaque et al., 2001).

Hodgson and Bachmann recently compared four approaches for $R D W$ algorithms (Hodgson and Bachmann, 2013). All four algorithms which are illustrated in 2.4 used the same steering parameters / manipulation thresholds determined in (Steinicke et al., 2010) and (Hodgson et al., 2011). The calculation of the particular rotation of the $V E$ is based on the algorithm presented in (Razzaque, 2005). The only difference among these four algorithms lay in where the user was being steered. The approach steer to center, which always steered the user to the center of the room outperformed the other three approaches, but the approach steer to orbit, which tried to keep the user on a circular path, was a close second and might outperform the first one in cases, in which users mostly walk on virtually straight paths with otherwise mostly orthogonal turns. Since Hodgson and Bachmann share Razzaques assumption that many scenarios naturally come with constraints like that (Razzaque et al., 2001) (e.g. hallways or streets), this was further investigated (Hodgson et al., 2014).

\subsection{The Human Locomotion Triple (HLT)}

In (Steinicke et al., 2008b) Steinicke et al. introduce the user's locomotion triple (in Steinicke et al. (2009) then named human locomotion triple (HLT)). The human locomotion triple (HLT) consists of three normalized vectors: $(s, u, w)$. The strafe vector $s$ is orthogonal to the walking direction and parallel to the walking plane, the up vector $u$ represents the tracked head orientation and the walk-direction vector $w$ represents the tracked direction of walk. Through the HLT, 


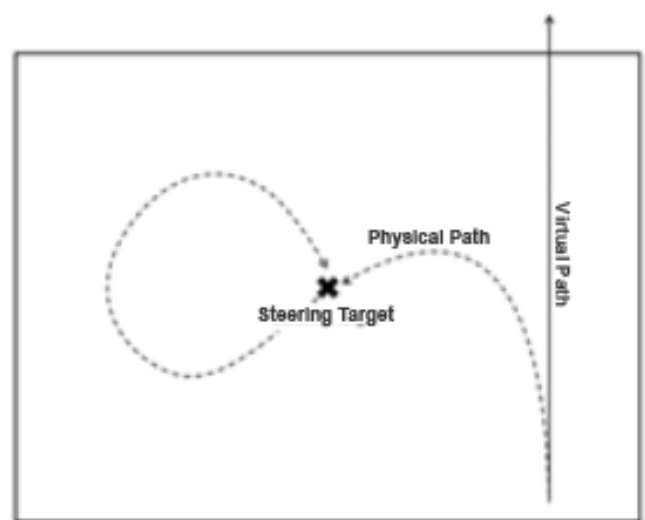

Steer To Center

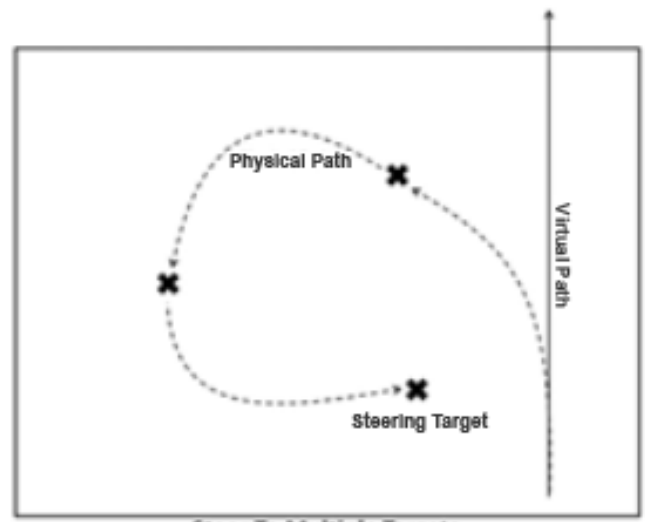

Steer To Multiple Targets

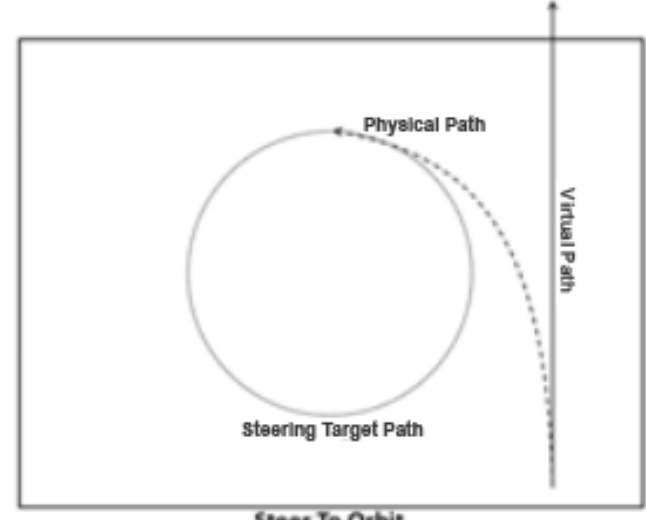

Steer To Orbit

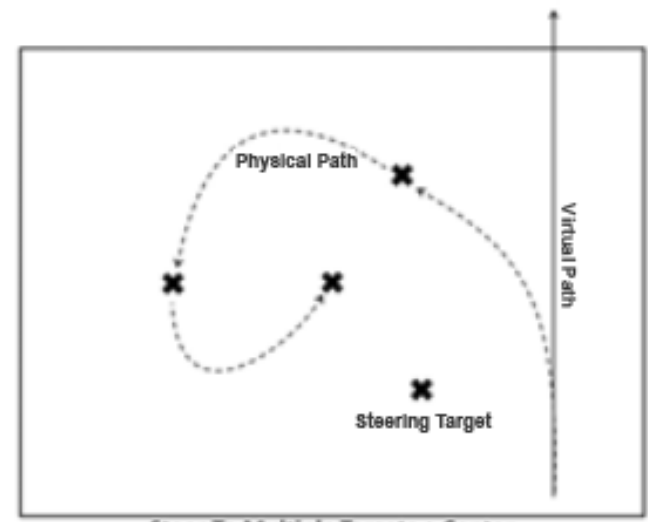

Steer To Multiple Targets + Center

Figure 2.4.: Illustrations of the four RDW algorithms tested in (Hodgson and Bachmann, 2013). Users were steered towards particular targets or onto a target path. Figure taken from (Hodgson and Bachmann, 2013).

manipulations can be applied to users' paths by various gains as described during the next sections.

\subsection{Gains to Manipulate the Users' Movements}

While the tracking system constantly provides up-to-date data for the users physical world position and orientation defined as $P_{\text {physical }}$ and $R_{\text {physical }}$, the translation is defined by

$$
T_{\text {physical }}=P_{\text {cur }}-P_{\text {pre }}
$$



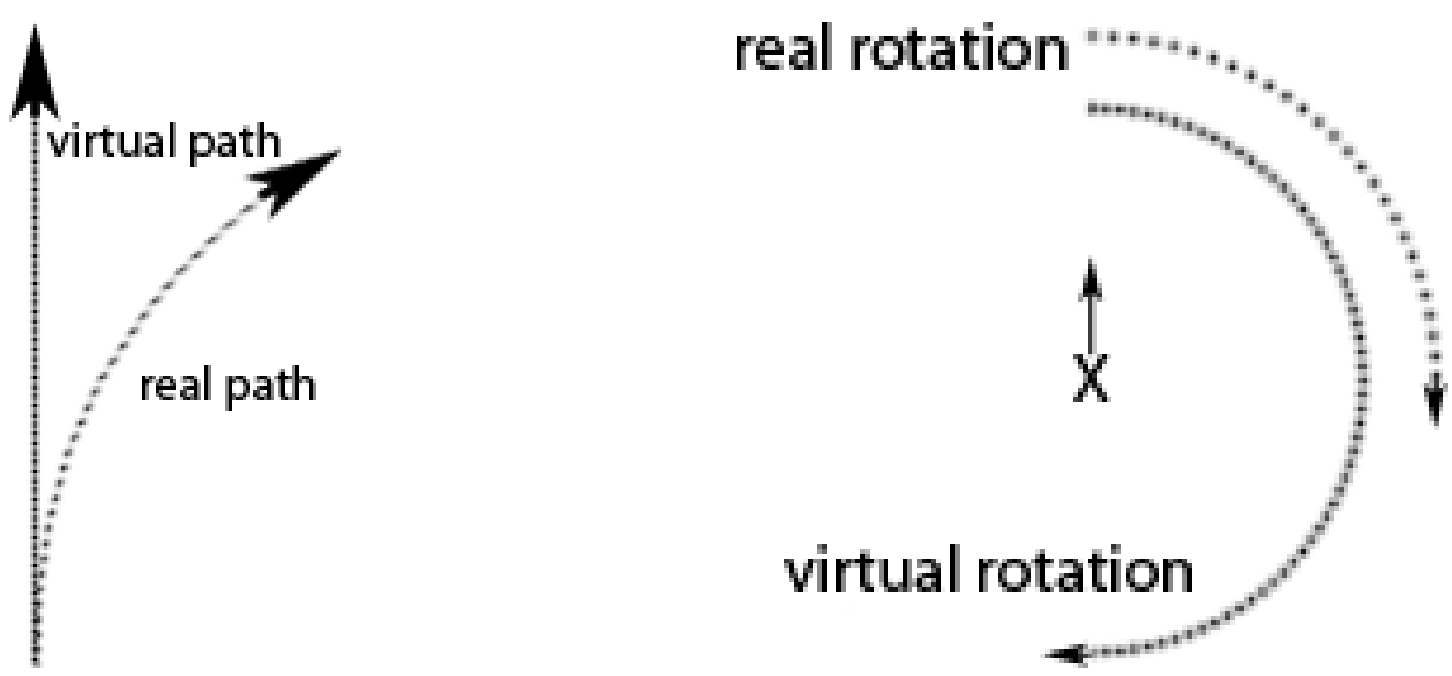

(a) The curvature gain bends a real path into a dis-(b) The rotation gain scales a rotation with the effect, torted virtual path. The user unknowingly walks on a curved path. that the virtual rotation is greater or smaller, than the real rotation.

Figure 2.5.: The curvature gain bends a path and the rotation gain scales a rotation.

where $P_{\text {cur }}$ is the current physical position and $P_{\text {pre }}$ the previous/last considered physical position. A translation gain $g_{T} \in \mathbb{R}^{3}$ is defined for each component of the $H L T: g_{T}[s], g_{T}[u], g_{T}[w]$ by

$$
g_{T}:=\frac{T_{\text {virtual }}}{T_{\text {physical }}}
$$

By such gains the mapping of physical world movements (in this last case translations) to virtual movements within a IVE can be scaled up or down, depending on the values of the gain. A $g_{T}<1$ would result in a smaller translation within the virtual world $\left(T_{\text {virtual }}\right)$ in respect to the tracked translation in the physical world $\left(T_{\text {physical }}\right)$, while a $g_{T}>1$ would result in a larger translation in the virtual world, and such enabling the users to cover a larger virtual distance. A $g_{T}=1$ would draw the physical world and the virtual world to scale as if no gain was applied at all.

In the same manner, Steinicke et al. introduce gains for rotation, curvature and displacement as well as time-dependent gains.

The curvature gain stimulates users to unknowingly walk an arc in the tracking area while walking on a straight line in the $V E$ even when they do not intentionally rotate (see figure 2.5a). A curvature gain is calculated by the radius $r$ of the complete circle defined by the curve:

$$
g_{C}=\frac{1}{r}
$$


The particular rotational manipulation $R_{\Delta}$ is then calculated by multiplying the physical translation with the curvature gain value:

$$
R_{\Delta}=T_{\text {physical }} \cdot g_{C}
$$

Five examples of bended paths with corresponding $g_{C}$ and $r$ are illustrated in figure 2.6.

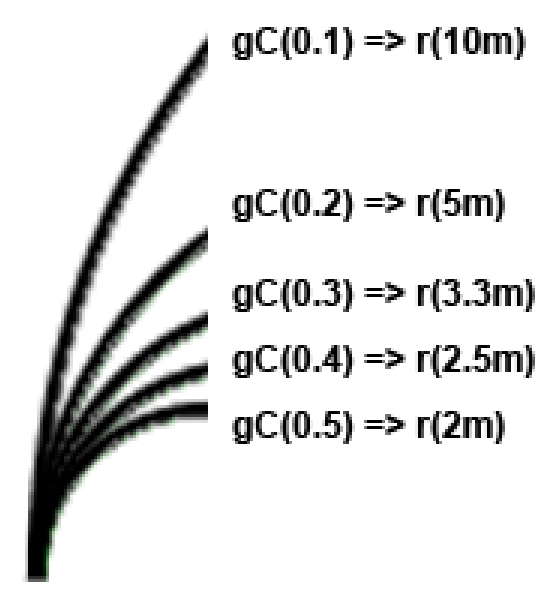

Figure 2.6.: Illustration of 5 different curves defined by the given values.

Rotation gains scale a users rotation to in- or decrease the amount of a users virtual rotation $R_{\text {virtual }}$ as illustrated in figure $2.5 \mathrm{~b}$, and are preferably calculated with the rotation of the users head $g_{R}[u]$ :

$$
g_{R}=\frac{R_{\text {physical }}-R_{\text {virtual }}}{R_{\text {physical }}}
$$

The particular rotational manipulation $R_{\Delta}$ is then calculated by multiplying the physical rotation with the rotation gain value:

$$
R_{\Delta}=R_{\text {physical }} \cdot g_{R}
$$

Figure $2.5 \mathrm{~b}$ illustrates a rotation gain with a value $g_{R}(-0.5)$, which down-scales a virtual rotation of $180^{\circ}$ to a physical rotation of only $90^{\circ}$.

Displacement gains, on the other hand, map physical world rotations into virtual world translations $\left(R_{\text {physical }}=>T_{\text {virtual }}\right)$. Time-dependent gains can be defined like all the other gains, though they are not triggered by physical world movements, but by time elapsed. One example of a time-dependent rotation gain would be to slowly turn the virtual world in either direction even while the user is standing perfectly still. By having the user perceive these 


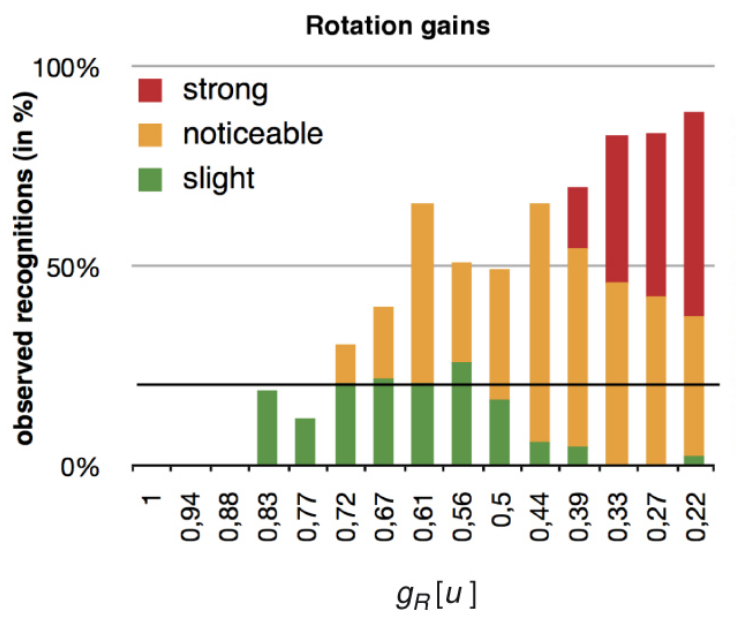

(a)

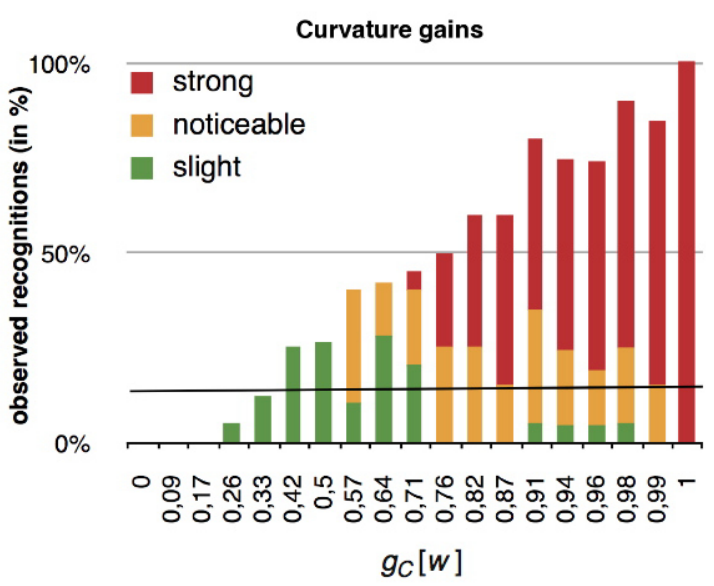

(b)

Figure 2.7.: Evaluation of the generic redirected walking concepts for (a) rotation gains $g_{R}[u]$ and (b) curvature gains $g_{C}[w]$. The bars indicate how much the users have perceived the manipulated walks. The horizontal lines indicate the detection thresholds. (Steinicke et al., 2008c, 2009)

rotations as self-motion, she subconsciously compensates for these rotations with physical world rotations. The result would be, that the user is slowly, unwillingly and unknowingly rotating on the spot.

\subsection{Experiments for Detecting Thresholds}

In March 2008 Steinicke et al. published results of a pilot study (Steinicke et al., 2008f) within a tracking range of $10 \mathrm{~m} \times 7 \mathrm{~m} \times 2.5 \mathrm{~m}$, in which they identified the following thresholds for $R D W$ without letting the users notice the manipulation:

- Rotations can be compressed or gained up to $30 \%$

- Distances can be downscaled to $15 \%$ and up-scaled to $45 \%$

- Users can be redirected to unknowingly walk on a circle with a radius as small as $3.3 \mathrm{~m}$

- Objects and the VE can be down-scaled to $38 \%$ and up-scaled to $45 \%$

The results of different experiments differ greatly though. Other experiments identified thresholds for manipulated rotations at $49 \%$ for up-scaling and $20 \%$ for down-scaling, as well 


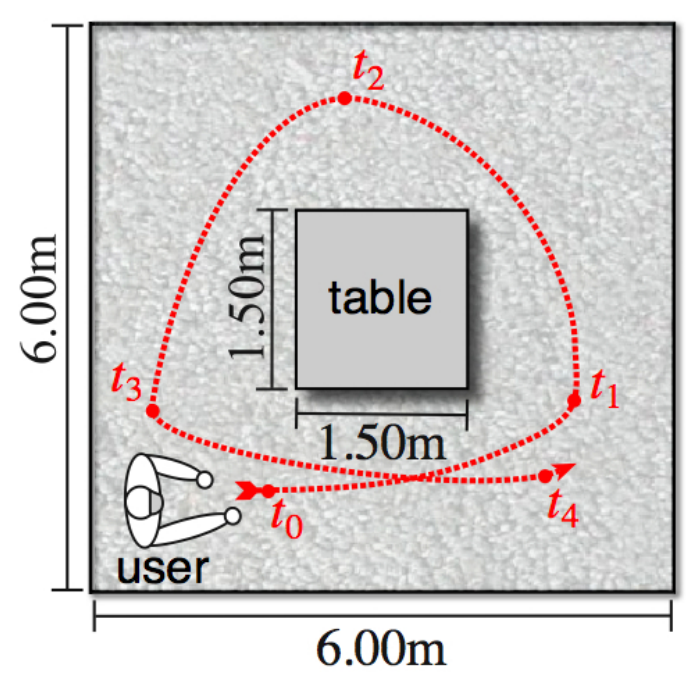

(a)

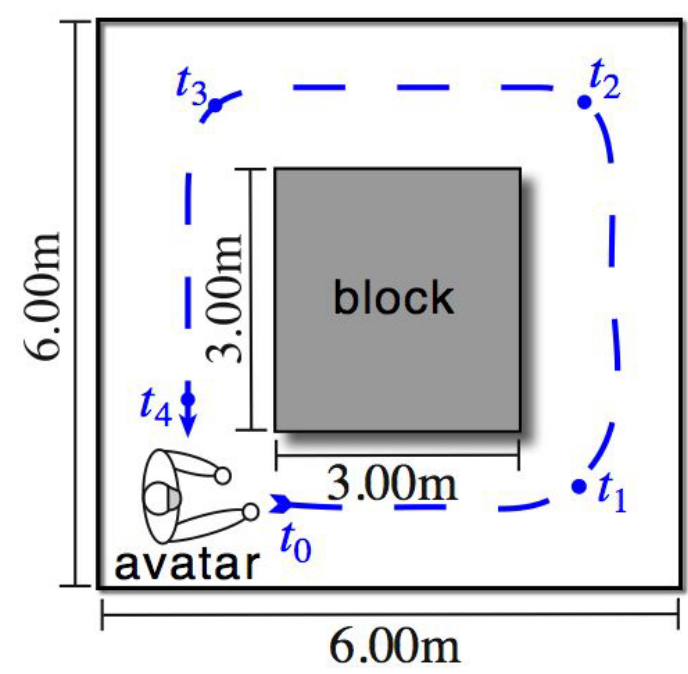

(b)

Figure 2.8.: Illustration of a user's path during a experiment in (Steinicke et al., 2008c) showing (left) path through the physical setup and (right) virtual path through the $V E$ and positions at different points in time $t_{0}, \ldots, t_{4}$. (Steinicke et al., 2008c)

as a radius for a curved path of 22 meters Steinicke et al. (2010), or 68\% for up-scaling, and $10 \%$ for down-scaling rotations Steinicke et al. (2008a). The differences in detection thresholds probably correlates with the attention, that the test subjects actively pay to the manipulations Steinicke et al. (2010).

Figure 2.7 shows whether and how strongly test subjects perceived the manipulation during rotational and walking tasks published in 2009 (Steinicke et al., 2009).

Figure 2.8 shows illustrations of a user's path during an experiment in Steinicke et al. (2008c). While the user is under the impression to have walked around a square block of 3 meters of edge length and ended up back at the starting point (figure $2.8 \mathrm{~b}$ ), the user was really directed around a square table of only 1.5 meters edge length and ended up well beyond the starting point (figure 2.8a) (Steinicke et al., 2009).

\subsection{Physical Obstacles}

A lot of the work of Steinicke et al. also includes the integration of physical objects to deliver passive haptics to virtual objects within the IVE (Steinicke et al., 2008b,e,f,g,d,c; Bruder et al., 2009), while they refer amongst others to (Insko, 2001; Insko et al., 2001; Kohli et al., 2005; Lindeman, 1999; Tachi et al., 1994). 


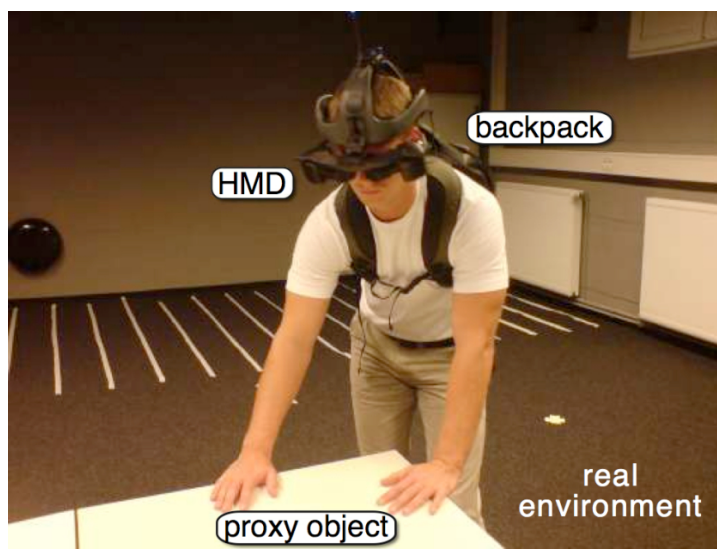

(a) A user touching a physical proxy object.

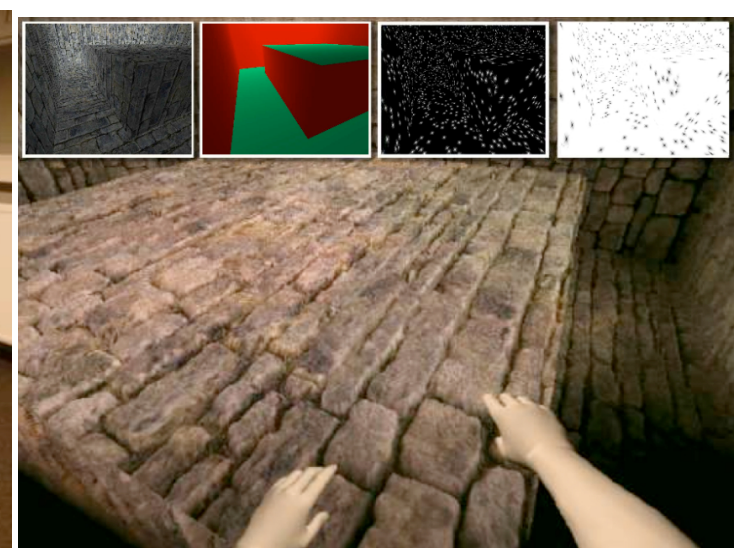

(b) The same user touching a virtual stone block at the same time.

Figure 2.9.: Two figures showing how a physical table serves as a block of stone in a VE (Steinicke et al., 2008c).

In general $R D W$ is used to guide a user, who is approaching an object in the $V E$ (figure $2.9 \mathrm{~b}$ ), to a physical proxy object within the tracking area (figure 2.9a), which then provides passive physical haptic for the virtual object. Due to $R D W$ scaling effects, the object does not have to have the same dimensions or exact haptic properties as the virtual object and can therefore be used for a wide variety of virtual objects. In other cases, the user is guided around the physical objects, to prevent collisions, using the same $R D W$-Techniques (Steinicke et al., 2008c).

\subsection{Velocity Dependency of $R D W$-Techniques}

The results of some studies suggested that the sensitivity to curvature manipulations might be dependent on the walking velocity (Cratty, 1966; Steinicke et al., 2008c, 2010). In 2012 Neth et al. investigated these assumptions (Neth et al., 2012). They conducted a total of 7200 trials with 12 participants. Per block of tests, each participant experienced 10 different curvature gains ranging from $0.005 \mathrm{~m}^{-1}$ to $0.05 \mathrm{~m}^{-1}$, corresponding to circular arcs with radii of 200 to $20 \mathrm{~m}$. Each gain was applied five times to the left and five times to the right. Each block was repeated three times for the three different walking velocities of $0.75,1.00$ and $1.25 \mathrm{~m} / \mathrm{s}$ and all three blocks per participant were repeated on another day in reverse order. All of these chosen walking velocities are slower than the average walking velocity of about $1.4 \mathrm{~m} / \mathrm{s}$ (Sekiya et al., 1996; Sreenivasa et al., 2008), because users tend to walk slower in VEs, than they would in reality (Mohler et al., 2007; Streuber et al., 2009; Souman et al., 2010). Figure 2.10 shows the identified detection thresholds for all participants per walking velocity, and a 
distinctive difference between the slowest tested walking velocity and the higher ones. This leads to the conclusion that users can be redirected more extremely when walking slower.

\subsection{Reorientation Technique (ROT)s and Distractors}

Even though multiple working $R D W$ algorithms have been implemented and tested with good results (Nitzsche et al., 2004; Razzaque et al., 2001, 2002; Razzaque, 2005; Su, 2007; Williams et al., 2006a, 2007; Steinicke et al., 2008c,f), it is theoretically impossible to design an algorithm, that will in all cases prevent the user from walking out of the tracking area without applying noticeable manipulations (Engel et al., 2008). So all of these systems also implement some kind of $R O T$ as an emergency procedure as a last resort to prevent the user from leaving the tracking area, when the algorithm is failing that task.

In (Razzaque et al., 2001) acoustic instructions were played in such a situation, that

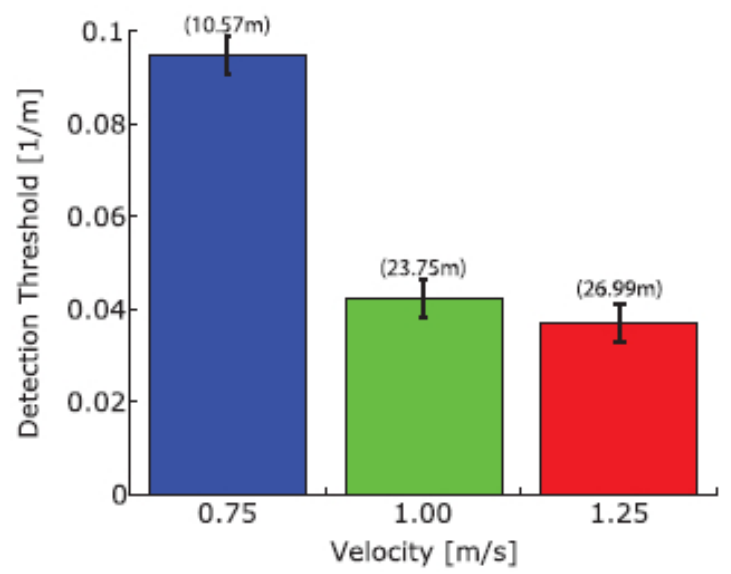

Figure 2.10.: Curvature detection thresholds across all participants (corresponding radius in brackets, bars denote standard error of the mean). (Neth et al., 2012) instructed the user to stop walking, then look left and right. These so caused head turns allowed the still running algorithm to apply additional rotational gains "hidden" within the self-motion of the user. Through these measures they were able to turn the users by more than 90 physical degrees and then letting the user continue walking in the the same virtual direction as before. Others asked the users to execute a $360^{\circ}$ turn instead of turning left and right (Williams et al., 2006b, 2007) or additionally used virtual stop signs (see Figure 2.11a) visually displayed in front of the user (Neth et al., 2012), but basically used the same procedure. In (Nitzsche et al., 2004; Su, 2007) the VE was just turned without regard to detection thresholds and the user, who was thus looking in another direction within the $V E$ had now to rotate herself, to be able to continue walking on the same virtual path as before. The price of all of these methods was paid in loss of immersiveness however. Peck et al. tested the use of a butterfly, that would enter the scene and fly closely in front of the users to distract her. The butterfly would then fly around the gazing user to animate a head rotation (Peck et al., 2009). Another likewise immersive approach features computer controlled 

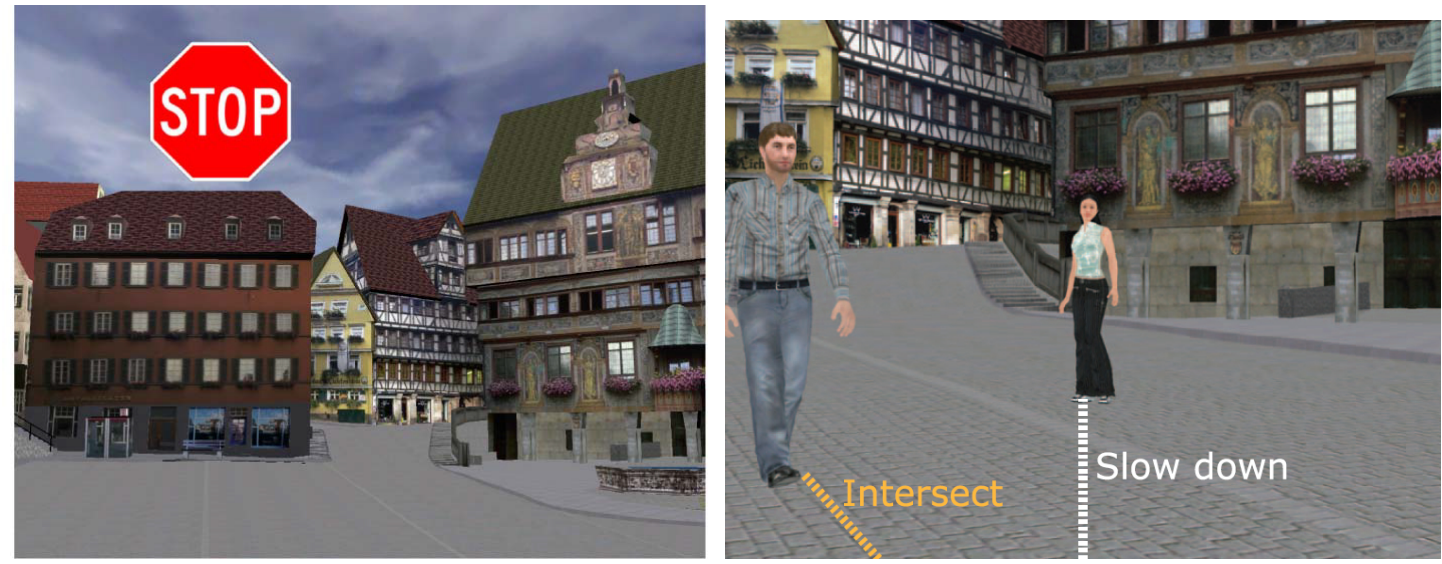

(a) A STOP sign as a means for a ROT signals the (b) NPCs intersecting the user to slow her down user to stop walking and perform some rotations so more redirection can be performed. and/or provoke (head) turns while she moves to avoid them.

Figure 2.11.: Two different approaches to ROTs to prevent a user from walking outside the tracking area. (Neth et al., 2012)

avatars (see Figure 2.11b) that would enter the scene in front of the user and intersect her path to slow her down and/or provoke (head) rotations, which can be exploited by the underlying $R D W$ algorithm.

\subsection{Non-Visual Redirected Walking by Acoustic Stimuli}

While a lot of research has been committed to $R D W$ during the last couple of years, almost all contributions are based upon the visualization of the $V E$ for primary stimuli. Some authors state that the acoustic factor helps users to adjust to the virtual world and that $R D W$ works best, when multiple cues, such as vestibular, visual and auditory, are consistent with each other, as this helps the user to perceive external-motion as self-motion (Lackner, 1977; Razzaque et al., 2001). Razzaque et al. indicate that a fully spatialized 3D sound model would be an important component to an IVE for $R D W$ (Razzaque et al., 2001), because the consistency of multiple cues for orientation and balance seem to help to interpret the rotation of the VE (external-motion) as self-motion Lackner (1977). Even though, the auditory aspect had been paid little attention so far (Serafin et al., 2013).

Razzaque et al. used circumaural stereo headphones to reduce the breaks in presences reported in Usoh et al. (1999) by blocking out lab noise, and to deliver spatialized environmental sounds and prerecorded instructions to the user, where the sounds were intended to be "plausible in both content and source location". The experimenter voice came from a virtual 
radio within the $V E$. The analysis of the effect of the sound was limited to the lack of negative comments by the users, regarding the auditory component of the virtual experience and not much specifics were given on the exact use (Razzaque et al., 2001).

Steinicke et al. used "[...] ambient city noise in the experiment such that an orientation by means of auditory feedback in the real world was not possible", but auditory cues were not used to directly aid the $R D W$ technique (Steinicke et al., 2008a,c, 2009, 2010). A lot of contributions do not mention auditory components at all.

To my knowledge currently Serafin et al. are the only ones, who really concentrated on the auditory component of $R D W$ techniques. They conducted two different experiments to determine thresholds for acoustic based $R D W$ techniques (Serafin et al., 2013). To that goal, they adapted two of the experiments conducted in (Steinicke et al., 2008a, 2010), to be used exclusively with auditory cues. Their experimental setup consisted of a surround system with 16 MB5A Dynaudio speakers in a circular array with a diameter of 7.1 meters and subjects wore an deactivated $H M D$ to block out their vision. The only audible feedback in both experiments was the sound of an alarm clock. The sound was delivered through the speaker array by the technique of vector base amplitude panning (VBAP), which, in such a setup, allows the placement of sounds within the circular array of speakers on a plane parallel to the ground level (Pulkki, 1997).

The first experiment tested the ability to detect rotation gains during rotations on the spot. The second experiment tested the detection of curvature gains while walking on a virtually straight line from one edge of the circular speaker array to a point roughly on the opposite side. Due to the limited space, only short distanced could be covered during each test.

During the first experiment the subjects were asked to turn on the spot towards the sound of the alarm clock. While they were turning, a rotation gain would rotate the alarm clock around the subjects. A rotation gain $>0$ would rotate the alarm clock in the same direction, the subject is turning and such making it necessary to turn further, to finally face the alarm clock. A rotation gain < 0 would have the opposite effect and result in a smaller physical rotation. When they perceived the sound as in front of them, they were asked whether they perceived the virtual rotation as larger (rotation gain $<0$ ) or smaller (rotation gain $>0$ ), than the physical rotation. The virtual rotation is perceived through auditory cues by locating the position of the sound source, while the physical rotation mainly by the vestibular and proprioception system. During the 22 subsequent trials per test subject, 11 different rotation gains were applied. Each gain was applied twice during the course of an experiment. For the evaluation Serafin et al. also oriented themselves at (Steinicke et al., 2010) and used a psychometric function to determine a bias for the point of subjective equality (PSE). The results for rotation gains are visualized in 


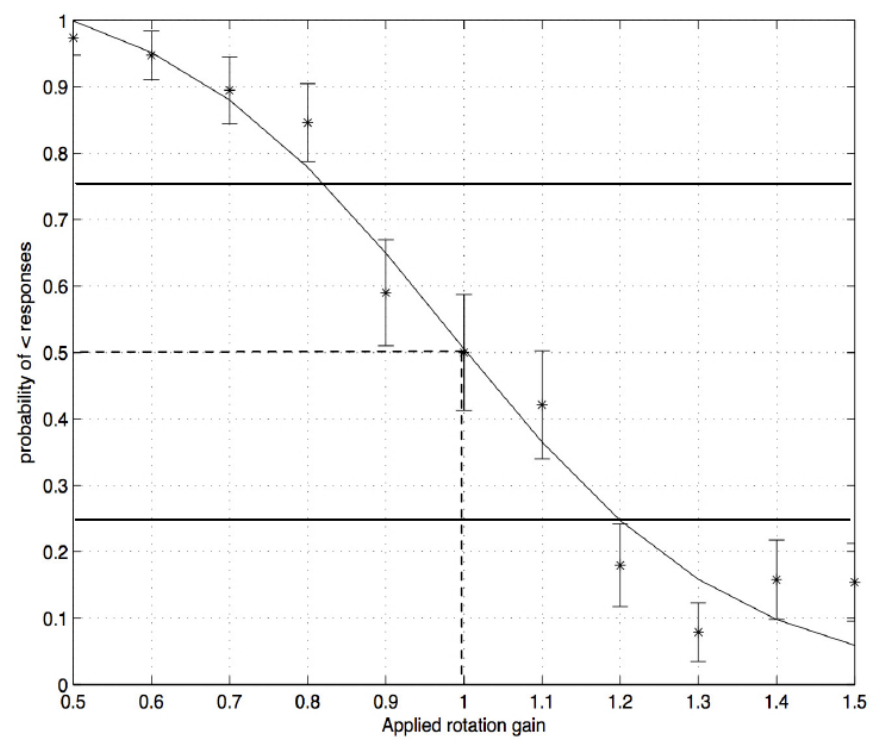

(a) Results of the discrimination between virtual and physical rotation. The $\mathrm{x}$-axis shows the applied rotation gain. The $\mathrm{y}$-axis shows the probability of estimating a virtual rotation smaller than the physical counterpart. (Serafin et al., 2013)

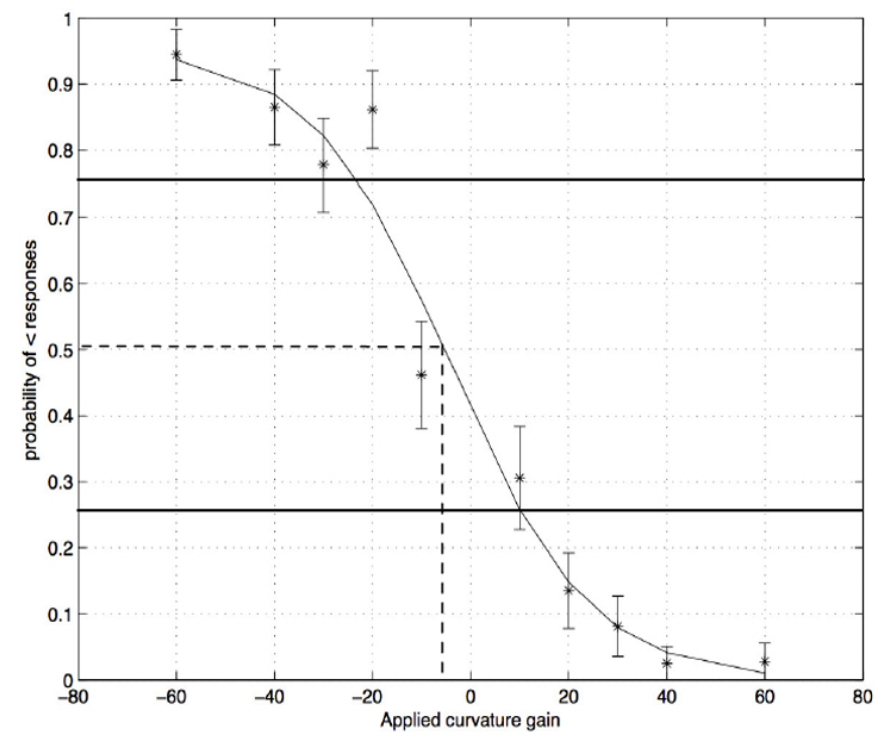

(b) Results for the discrimination of path curvature. The $\mathrm{x}$-axis shows the applied curvature gain which bends the walked path either to the left or the right. The y-axis shows the proportion of subjects "left" responses. (Serafin et al., 2013)

Figure 2.12.: Two figures showing the results of the two-alternative forced-choice (2AFC) experiments in (Serafin et al., 2013). In the $2 A F C$ experiment, subjects had to give one of two possible answers after each test. For (a) it was "greater" or "smaller". For (b) is was "left" or "right". These figures show the proportion of one of these answers to the other one. 
figure 2.12a. The PSE, where subjects perceived the physical and virtual rotation as equal, was determined at 1 . Serafin et al. also chose an outbalance of $75 \%$ to $25 \%$ of the given answers as the detection threshold and these thresholds were reached at gains of 0.82 for greater and 1.2 for smaller responses. This led them to the conclusion, that users can not reliably distinguish between a $90^{\circ}$ physical rotation and a virtual rotation between $75^{\circ}$ and $109^{\circ}$. So users can be turned $20 \%$ more or $18 \%$ less than the perceived virtual rotation. This range is smaller than the one determined in (Steinicke et al., 2010), which can be attributed to the fact, that

"[...] vision generally is considered superior to audition when it comes to the estimation of spatial location of objects." Goldstein (Goldstein, 2010) cited by Serafin et al. (Serafin et al., 2013)

In other words, visual cues dominate vestibular, proprioceptive, etc. cues more, than auditory cues would and therefore discrepancies between visual and other cues would be accepted to a higher degree.

During the second experiment users were asked to walk on a straight line towards the alarm clock. During their movement 10 different curvature gains were applied (each one twice), which led them on an arced physical path and users were asked whether and at which threshold they notice the direction of the bended path reliably. For this experiment the curvature gain value was defined as the degree the scene rotated after the test subjects walked the whole path of 5 meters. The conversion between this representation and the one used in the work presented here and i. e. in figure 2.7 is illustrated in equation A.7 During this experiment the PSE was determined at a curvature gain of -5 . The detection thresholds of $75 \%$ were reached at gains of -25 and $10^{1}$ (Serafin et al., 2013). 25 is roughly equivalent to a circle with a radius of 11.45 meters.

\subsection{Cyber Sickness}

In 1993 Kennedy et al. introduced the simulator sickness questionnaire (SSQ) (Kennedy et al., 1993), which they derived from the motion sickness questionnaire (MSQ), to measure sickness induced by the use of high-fidelity visual simulators. In 1994 Michael Moshell and Dan Mapes put their work on $R D W$ to rest, because they could not elude the problem of what they identified to be simulator sickness.

In 1997 Stanney et al. published a paper to differentiate between simulator sickness and cyber sickness. While both share most of their symptoms, in cyber sickness disorientation is

\footnotetext{
${ }^{1} \mathrm{~S}$. Serafin confirmed in personal correspondence that a mistake slipped into the textual representation of the results. +10 is correct (as the corresponding plot illustrates) instead of +30 .
} 
the predominant factor, while in simulator sickness it is nausea (Stanney et al., 1997). In recent publications, the terms are often used synonymous, however.

Even though Moshell and Mapes stated their problem as primary technical (slow and imprecise tracking systems) (Razzaque et al., 2001), the problem persists as a result of the fundamental principle of $R D W$. Motion sickness, simulator sickness and cyber sickness are all caused by the reception of incongruent cues for movement and/or orientation. On a ship, below deck, the visual cues may not indicate any external-motion at all, while the sense of balance reports a swaying. The same goes for cars, trains, flight- and space simulators, etc. This discrepancy can cause different kinds of sickness symptoms (Kolasinski, 1995). While most simulators, such as high-end flight simulators for pilot training, aim to deliver coherent cues for all senses, the $R D W$ technique is based on the discrepancy between different cues. Assuming the vestibular and proprioception systems will always report correctly, the visual and/or auditory cues are willfully manipulated into reporting something different. Due to this fact, the consideration and measurement of cyber sickness is part of most experiments regarding $R D W$.

The symptoms measured by the $S S Q$ are listed in table 2.1. The table also shows, which aspects of the overall score are influenced by each symptom. The questionnaire registers four different levels of severity for each symptom that the test subject might experience. None, slight, moderate and severe. These are matched to multipliers of $0,1,2$ and 3 in this order. Together they result in sub scores for each aspect. To calculate the total and sub scores, the multipliers of table 2.2 are used. The total score is calculated by summarizing the sub scores $\mathrm{N}, \mathrm{O}$ and D and multiplying the result with 3.74. The sub scores Nausea, Oculomotor and Disorientation are calculated by multiplying $\mathrm{N}, \mathrm{O}$, and $\mathrm{D}$ with the respective multipliers in table 2.2. The severity of each symptom is registered right before and right after the use of an $V E$ and the difference of the scores give insight about the influence of the use of that particular $V E$. 


$\begin{array}{lccc}\text { Symptoms } & \text { Nausea } & \text { Oculomotor } & \text { Disorientation } \\ \text { General discomfort } & 1 & 1 & \\ \text { Fatigue } & & 1 & \\ \text { Headache } & & 1 & \\ \text { Eye strain } & & 1 & 1 \\ \text { Difficulty focusing } & & 1 & \\ \text { Increased salivation } & 1 & & 1 \\ \text { Sweating } & 1 & & 1 \\ \text { Nausea } & 1 & & 1 \\ \text { Difficulty concentrating } & 1 & 1 & 1 \\ \text { Fullness of head } & & & 1 \\ \text { Blurred vision } & & 1 & \\ \text { Dizzy (eyes open) } & & & \\ \text { Dizzy (eyes closed) } & & & \\ \text { Vertigo } & & & \\ \text { Stomach awareness } & 1 & & \\ \text { Burping } & 1 & & \\ \text { Sum } & & \mathrm{O} & \end{array}$

Table 2.1.: Weights for Symptoms of the Simulator Sickness Questionnaire (SSQ). The severity value the subject reported $(0,1,2,3$ or 4$)$ is multiplied by the corresponding weight ( 0 or 1$)$ to receive the value for that specific symptom.

$\begin{array}{lll}\text { Nausea } & =\mathrm{N}^{2} & \cdot 9.54 \\ \text { Oculomotor } & =\mathrm{O}^{2} & \cdot 7.54 \\ \text { Disorientation } & =\mathrm{D}^{2} & \cdot 13.92 \\ \text { Total } & =\text { Sum } & \cdot 3.74\end{array}$

Table 2.2.: Calculation of the Simulator Sickness Questionnaire (SSQ) score.

\footnotetext{
${ }^{2}$ See table 2.1
} 


\section{Wave Field Synthesis (WFS)}

This chapter will give a short insight into general wave field synthesis (WFS) and into the Fouraudio's ${ }^{1}$ wave field synthesis system (WFS system), which is installed in the laboratory in which the experiments were conducted. The focus of this chapter will be on the principles, architecture and interfaces rather than on the rendering.

\subsection{Motivation}

A WFS system is supposed to generate an acoustic environment as real as possible without the inherent generation of a sweet $\operatorname{spot}^{2}$. The quality of the acoustic environment shall be perceived with consistent quality regardless of the position within the physical WFS area (Baalman, 2008, p. 11). The system abstracts from the speakers and general physical layout of it's setup to deliver the possibility to position and move virtual sound sources in real time to any position. While simpler sound systems pan the volume between channels/speakers to generate phantom sound sources at specific locations between those speakers (see "stereo panning") (Tzanetakis et al., 2010; Andresen, 2002; Floros and Tatlas, 2011), a WFS system renders a synthetic wave field, whose form is close to a real wave field with its origin at the location of the corresponding virtual sound source. Virtual sound sources are a WFS systems version of a phantom sound source of stereo systems and represent origins of synthetic wave fields.

Possible applications could be found in creating specific test environments or in high end entertainment systems like cinemas. 


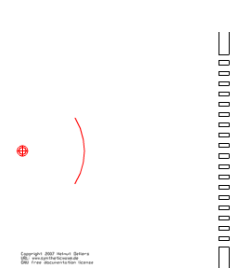

(a) Its being computed, how a real wave would expand...

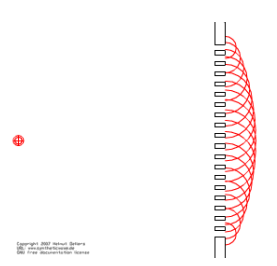

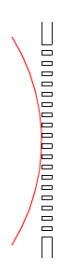

(b) ...until it meets the positions of the speakers of the WFS system.

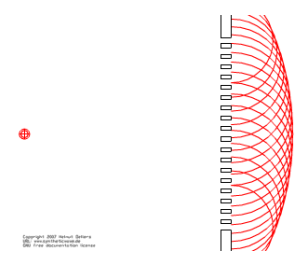

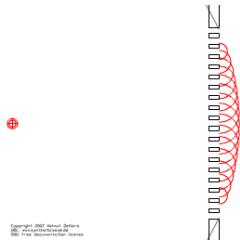

(c) Each channel creates an elementary wave with the corresponding delay and amplitude...

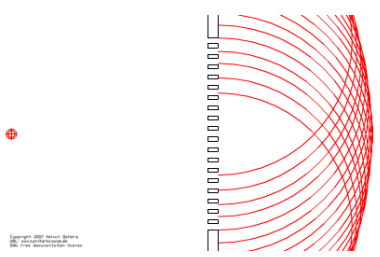

(d) ...which start to overlap (e) ...to form the synthetic sound (f) The elementary waves form each other shortly after... wave. the synthetic sound wave.

Figure 3.1.: The creation of a synthetic sound wave (synthetic wave audio products $\mathrm{GmbH}$, 2015).

\subsection{The Principle}

This section will give a brief overview over the principle of WFS in two dimensional space. For a more detailed description I recommend the dissertation of Marije Baalman (Baalman, 2008).

The WFS uses huygens principle, which states for light waves that every point of a wavefront may be the origin of a new elementary wave (Huygens and Blay, 1920). This principle can also be applied to other kinds of waves like sound or water. The new wave is formed by all the overlapping elementary waves, as illustrated in figure $3.1 \mathrm{c}-3.1 \mathrm{f}$.

For the following explanation please refer to figure 3.1. Imagine a massive but thin wall, which is perforated by vertical slits. On one side of the wall is a real sound source which emits its sound waves in an even spheric manner. The wall blocks the expansion of the sound wave but for the area of the slits. On the other side of the wall, each slit is perceived as the origin of a new elementary wave, which is the fraction of the original sound wave, which is passing through this particular slit. A fraction of each elementary wave will behave, as if there was no wall and no slits. Shortly after the creation of these elementary waves (depending on the

\footnotetext{
${ }^{1}$ http://www.fouraudio.com/

${ }^{2}$ Stereo- or surround systems are always configured towards one specific spot (the sweet spot) within the room. Only on this position will the sound system deliver, what the sound designer intended and the quality will decrease with increased distance to this spot.
} 
distance between the slits/origins of elementary waves), they will start to overlap with their neighbours and then neighbours neighbours and so on. While overlapping, the fraction of each wave, which was unaffected by the wall, will be the leading part of that elementary wave, and will not be overlapped by any other elementary wave at any time. All the leading fractions will form the front of the new sound wave through which the expansion pattern of the original wave is almost maintained. The only difference is a small bumpiness of the wave front, whose severity is determined by the distance of the slits in the wall and will not be perceived, as long as it is not too severe. Through the perpetuation of the form of the wave front, the origin of the original sound wave can still be located from the other side of the wall.

The WFS system merely replaces the slits of the formerly mentioned wall by speakers and the real sound source by a virtual sound source. The fictional slits and the channels of the WFS system are arranged in a bijective relation. The extension of the virtual sound wave is implicitly calculated by calculating a delay for each channel (the distance between the virtual sound source and the channel - sound velocity) and the decrease of amplitude for the same distance. Each channel will emit the sound of the virtual sound source with its own delay and amplitude, and such act like the formerly described fictional slits (figure 3.1c). The physical elementary waves together form the synthetic wave (figures $3.1 \mathrm{c}-3.1 \mathrm{f}$ ).

\subsection{Properties of Virtual Sound Sources}

The most important property of a virtual sound source is, in most cases, its position. Most commonly a virtual sound source would be a point sound source, which emits its sound in an even circular manner. WFS systems may, however, also implement linear sound sources, which emit directed linear waves. For linear sound sources not the position, but the orientation is relevant. Besides the type, position, orientation and an ID for referencing virtual sound sources might have (amongst others) secondary attributes like a name and colour for visualisation.

\subsection{Technology}

The WFS system of Four Audio consists of the WFS server, the WFS mac (control computer), multiple WFS nodes and audio modules (Goertz et al., 2007). Each audio module consists of eight channels. The quantity of WFS nodes and audio modules depends on the size of the physical WFS area. The WFS laboratory at the University of Applied Sciences Hamburg has two nodes with 13 audio modules each, which amounts to 208 channels. 


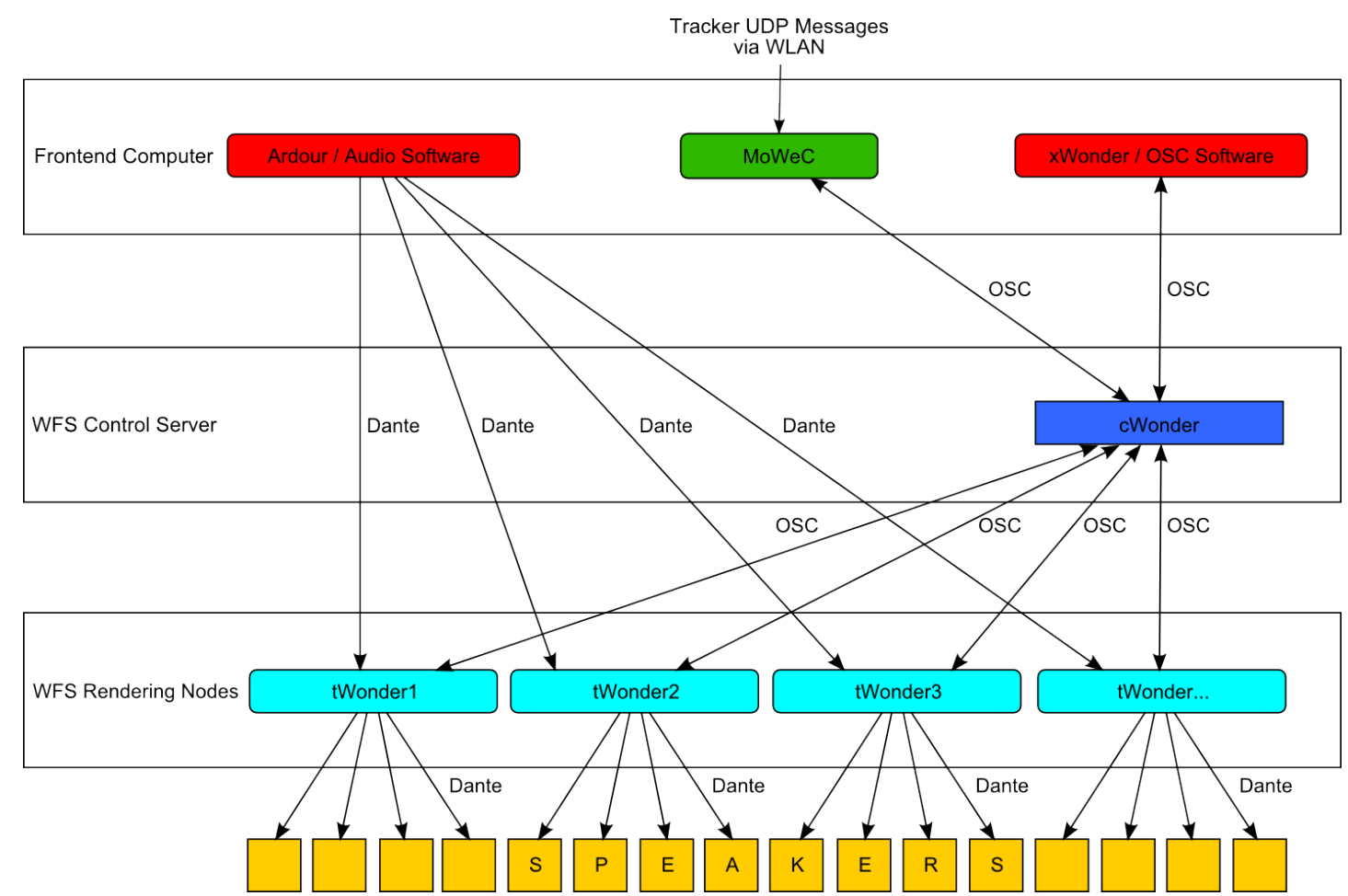

Figure 3.2.: Layout of the WFS system based on (Four Audio GmbH, 2011).

This laboratory runs the open source software Wave field synthesis of new dimensions of electronic music in realtime (WONDER) $)^{3}$ (Baalman and Plewe, 2005; Baalman, 2008). It consists of multiple distributed modules, which communicate over LAN via open sound control (OSC) messages (Baalman et al., 2007). Figure 3.2 illustrates the layout of the sWONDER components and hardware modules (Baalman, 2008, P. 50ff).

The control computer functions as the user interface. It offers AppleScripts to start and stop the WFS system, by starting corresponding scripts on the server and nodes. All programs, that generate or play back sounds for the WFS system, run on the control computer. The type of audio program is not restricted. Mighty digital audio workstations (DAWs) work as well as simple MP3 players. The control computer is the only one that should be connected to external systems, and it also runs the $x$ WONDER module which offers a graphical user interface (GUI) (figure 3.3) to control the virtual sound sources, load and save projects etc.

cWONDER is the central communication module of the WFS system, which runs on the WFS server. OSC messages are used to propagate control commands. cWONDER forwards all incoming OSC messages to all registered recipients. Other components deliver all outgoing

\footnotetext{
${ }^{3}$ http://sourceforge.net/projects/swonder
} 
OSC messages only to $c$ WONDER after registering themselves at start up (Baalman, 2008, P. 53).

tWONDER is the rendering unit. It is a jack ${ }^{4}$ client with an input port for each virtual sound source, and an output port for each audio channel. It uses the position data of virtual sound sources supplied by $c W O N D E R$ and fixed position and orientation date for each audio module, to calculate the delay and amplitude for each channel. Multiple $t$ WONDER instances distribute the computation load between nodes (Baalman, 2008, P. 55).

\subsection{Operating Options}

The only computer of the WFS system which is operated directly at runtime is the control computer, but other computers can be connected to the OSC network on demand, to also send OSC messages to $c W O N D E R$ and therefore control the WFS system.

\subsubsection{Open Sound Control (OSC)}

All communication between WONDER instances is conducted via OSC messages. OSC is a network protocol that had been designed to provide real time communication of control commands between various multimedia devices specifically for electronic music (The Center For New Music and Audio Technology (CNMAT), 2015). All components of WONDER use OSC messages for any communication and so do those that allow interaction with the system and any components that might be added at a later time. By easily registering new components to the central communication module $c W O N D E R$ via a single OSC message, it is very easy to add further components to the system at a later time. Whether they just listen or inject OSC messages on their own does not matter. Through this system, any programs that are capable of OSC communication are theoretically capable of interacting with the WFS system.

Some examples for operation options via OSC will be given in this chapter.

\subsection{2. $x$ WONDER}

$x W O N D E R$ is the GUI component of WONDER. It supports loading and saving of projects, creating and deleting of virtual sound source, changing the position of virtual sound source via drag and drop and changing the angle, type, name, ID and colour of each virtual sound source. All changes made in $x W O N D E R$ are transmitted to the central communication module cWONDER via $O S C$ messages.

\footnotetext{
${ }^{4}$ http://jackaudio.org/
} 


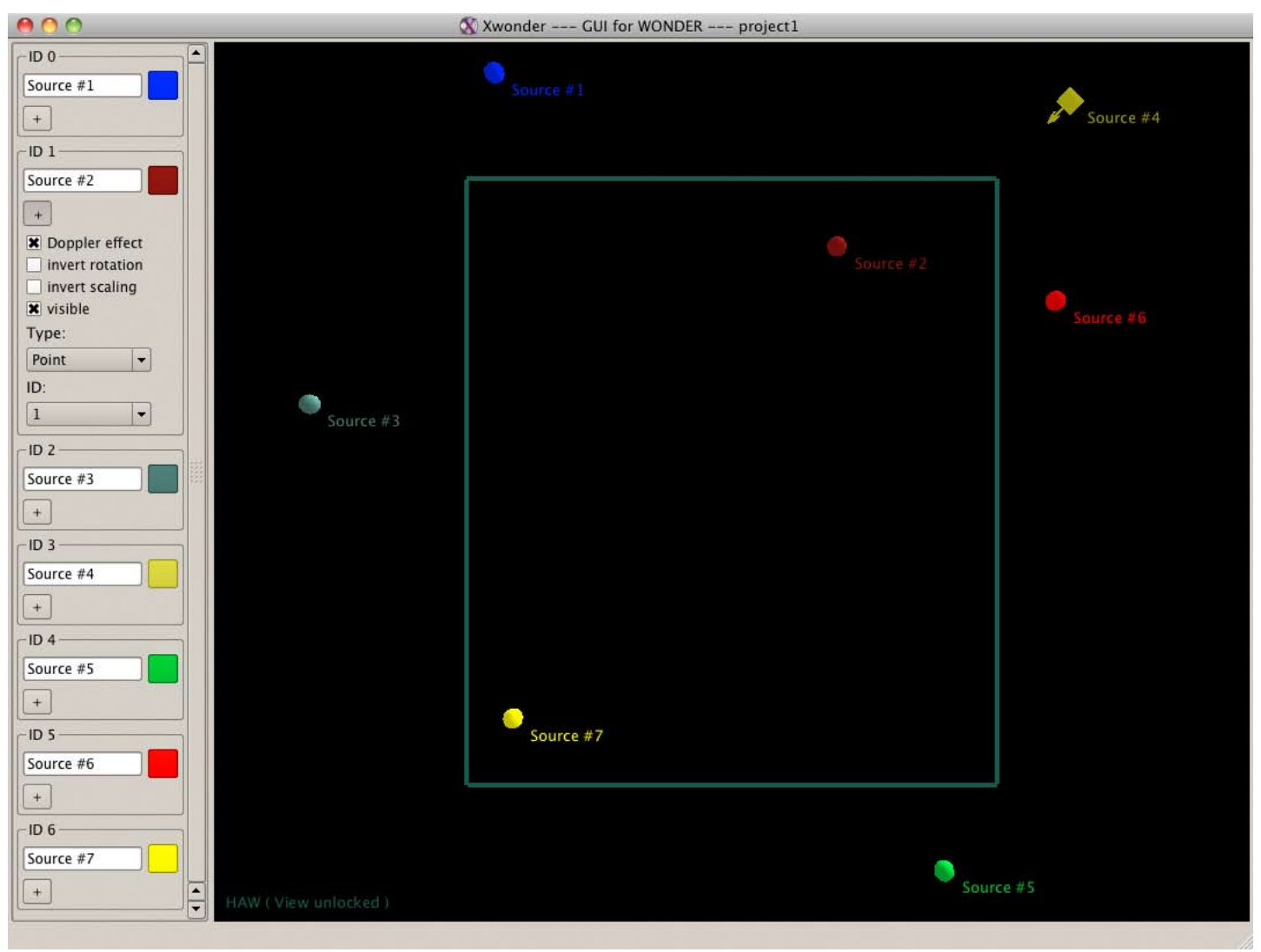

Figure 3.3.: Exemplary WFS layout displayed by $x W O N D E R$.

All changes to the system that are simultaneously executed by other programs, will be propagated by $c W O N D E R$ to $x W O N D E R$ via OSC messages. That way $x W O N D E R$ can also be used just to monitor the system setup, while using other components to make changes. Figure 3.3 shows $x$ WONDERs illustration of an exemplary WFS setup.

\subsubsection{SuperCollider}

SuperCollider ${ }^{5}$ is a real time audio synthesis programming language. SuperCollider provides a simple solution, to create and send an OSC message within one line of code. The following line of SuperCollider code creates an OSC message to set the position of the virtual sound source with $\mathrm{ID}=0$ to $\mathrm{X}=3.5$ and $\mathrm{Y}=-1.5$ and sends the message to the central communication module $c W O N D E R$ (port $=58100)$, which is running on the WFS server $(\mathrm{IP}=192.168 .3 .1)$ :

\footnotetext{
${ }^{5} \mathrm{http}: / /$ www.audiosynth.com/
} 
NetAddr("192.168.3.1", 58100)

.sendMsg("/WONDER/source/position", $0,3.5,-1.5)$; 


\section{Tracking Methods and Systems}

This chapter will give a brief overview over tracking methods and systems without being exhaustive. It will illustrate a few different tracking methods with its pros and cons and will finally focus on the ART tracker, which was used for this work.

\subsection{Tracking Systems in General}

Tracking systems capture the position and possibly the orientation of objects within a defined physical space. There is a vast amount of entirely different approaches to tracking methods, while each approach has its own pros and cons which can lie (amongst others) within accuracy, real time capability, robustness, size of tracking area, cost or flexibility.

\subsection{Differences Between Tracking Methods and Systems}

This section will illustrate the main differences between different tracking methods.

There are marker-based and marker-less tracking methods. Marker-based tracking methods track specially designed objects, while marker-less methods track natural objects or persons themselves. Markers are further differentiated into active and passive markers. Active markers generate some kind of signal like light or radio etc. and generally need electricity to do so. Passive markers are not electronic and only have passive properties like a distinctive geometry, colours, reflections etc. In marker-based tracking systems, every object, which shall be tracked, has to be equipped with at least one marker. Marker-less objects would be invisible to the tracking system.

Marker-less methods have the advantage, that they require less preparation and provide more flexibility, but often at the price of less accuracy and/or more delay and a lower refresh rate due to higher computing load. There are a lot of different marker-less tracking methods. Cameras can save their pictures, identify prominent characteristics (features) and scan following pictures for these features, and therefore track an object's movement. Other systems only register infrared light. For passive marker methods, those systems would have markers, that are equipped with reflecting areas, and the system itself would send out infrared light to generate 
strong reflections. As long as multiple cameras register one and the same reflection, the system can easily calculate the position of that reflection. An infrared tracking system with active markers would not have to send infrared light itself to generate reflections, but the markers would instead generate infrared light themselves. Other systems project a distinctive infrared pattern on the scene, while a camera films how the pattern is distorted by different depths of the scene. The system then calculates a complete depth map of the scene to identify and distinguish between different objects, and thus being able to track them. Some marker-less as well as marker-based systems use the time light or other data take to travel to a destination and back (light -> passive, radio signals -> active) to calculate the distance to the object, and then by triangulating calculate the position of the object.

Some systems register the position of objects in three, and other only in two or one dimension. Table 4.1 gives some examples.

\begin{tabular}{ll||l|l}
\multicolumn{1}{c||}{ Method $\backslash$ Dimension } & $2 \mathrm{D}$ & 3D \\
\hline \hline \multirow{2}{*}{ Marker-based } & (Active) & Face motion tracking & $\begin{array}{l}\text { A.R.T. LED targets (Optical Biangulation) } \\
\text { GPS (Triangulation) }\end{array}$ \\
\cline { 2 - 4 } & \multirow{2}{*}{ (Passive) $)$} & $\begin{array}{l}\text { RGB feature tracking } \\
\text { (Predefined Features) }\end{array}$ & $\begin{array}{l}\text { A.R.T. Reflector targets } \\
\text { (Optical Biangulation) }\end{array}$ \\
\hline \multirow{2}{*}{ Marker-less } & $\begin{array}{l}\text { RGB feature tracking } \\
\text { (Dynamic Features) }\end{array}$ & Microsoft Kinect ${ }^{1}$ (Light Coding)
\end{tabular}

Table 4.1.: Examples for 2D and 3D marker-based and marker-less systems.

\subsection{Requirements of the Redirected Walking Component}

The requirements a redirected walking application has towards its tracking system are moderately high. Razzaque et al. wrote in (Razzaque et al., 2001) that Michael Moshell and Dan Mapes had to abandon their work on $R D W$ in 1994, because they could not elude the problem of simulator sickness as a result of limitations of the tracking system. Especially a high latency would be a problem, since the users movements are transferred into the redirected walking application, and if the latency would be to high, the user would notice that the application is smearing her movements. Breaks in presence, simulator sickness and a higher detection rate of the $R D W$ manipulations are probable consequences. A high frame rate is desirable to achieve smooth movements. The precision should not be off by more then a couple centimetres/degrees, but more precision will always result in a better result.

The system has to be able to track the position as well as the orientation of objects and must be able to identify unique objects. 


\section{The Laboratory}

This chapter will give a brief overview of the laboratory in which the experiment was conducted, and the software that had been implemented. An illustration of the laboratory can be found in figure 5.1 and a more detailed description in (Fohl, 2013).

The area of the WFS system is defined by the speaker arrays and covers roughly $5 \times 6$ meters. The height of the lower edges of the speakers is just over 2 meters. The reproduction component of the system consists of 26 speaker modules which contain 676 single speakers equally divided amongst 208 channels. The distance between the centers of the channels within each speaker array is $10 \mathrm{~cm}$. All modules are slightly tilted downwards, and both of the 6 meter arrays and one of the 5 meter arrays is backed by a wall of the room.

The tracking area is defined by six infrared cameras, which are arranged in a square formation of $4 \times 4$ meters parallel to the ground in an elevation of about 2.5 meters with the two extra cameras mounted below two adjoining corners in about 1.5 meters height. All cameras are roughly aligned towards the middle of the tracking area. Due to the range of the cameras, the tracking area is slightly larger than the $4 \times 4$ square but the tracking is most robust within these boundaries.

The WFS terminal is located in one of the corners of the room. The terminal serves as the primary user interface for the WFS system. Within are also all instances and programs for playing back sounds, propagating commands and rendering for the speaker modules. The main DAWs in use are Ardour ${ }^{1}$ and Cubase ${ }^{2}$.

\subsection{Motion Tracker-Wave Field Synthesis-Connector (MoWeC)}

The $\mathrm{MoWeC}$ is described in detail and in German language in (Nogalski, 2012) and had also been presented on the 13th International Conference on New Interfaces for Musical Expression 2013 in Daejeon, Korea Republic (Fohl and Nogalski, 2013). Its processing sequence is illustrated in figure 5.2.

\footnotetext{
${ }^{1}$ https://ardour.org/

${ }^{2}$ www.steinberg.net/de/products/cubase/
} 


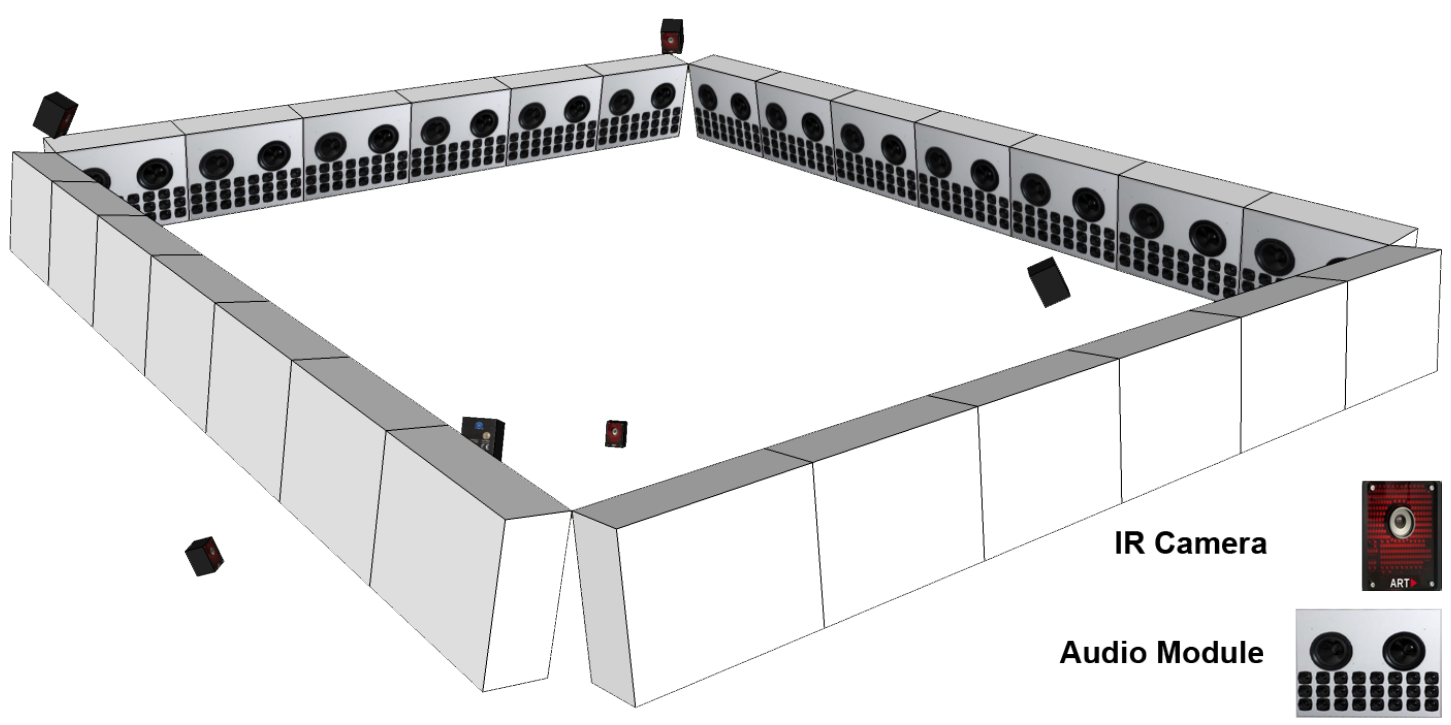

Figure 5.1.: Layout of the laboratory with 26 audio modules and 6 infrared cameras.

The $\mathrm{MoWeC}$ is implemented in Java 1.6 and driven by incoming network packages carrying tracking data, which are forwarded by the TrackerListener Application. This application provides a loose coupling between the $\mathrm{MoWeC}$ and its tracking system, while supplying the tracking data. The tracking data is then parsed and converted into the internal data type MoWeC source. The MoWeC is designed to work with these MoWeC sources.

The center of the $\mathrm{MoWeC}$ is defined by the optional components section. This is where the application logics can be implemented. Each optional component provides an application logic or a part of the overall application logic. Multiple optional components can run the same time, as long as they do not object each other logically. Optional components are modifying the MoWeC sources in respect of their position and orientation parameters etc..

After the optional component section, the MoWeC sources are converted into the target coordinate system of the WFS system. After some screening, to reduce network traffic and to relieve the WFS system of unnecessary small changes and resulting computation load, OSC messages are created to deliver all changes to the WFS system and DAWs.

Simultaneously the OSC listener receives all changes to the WFS system by previously registering at its server. This incoming data is converted into the internal coordinate system of the $M o W e C$ and provided to the optional components. 


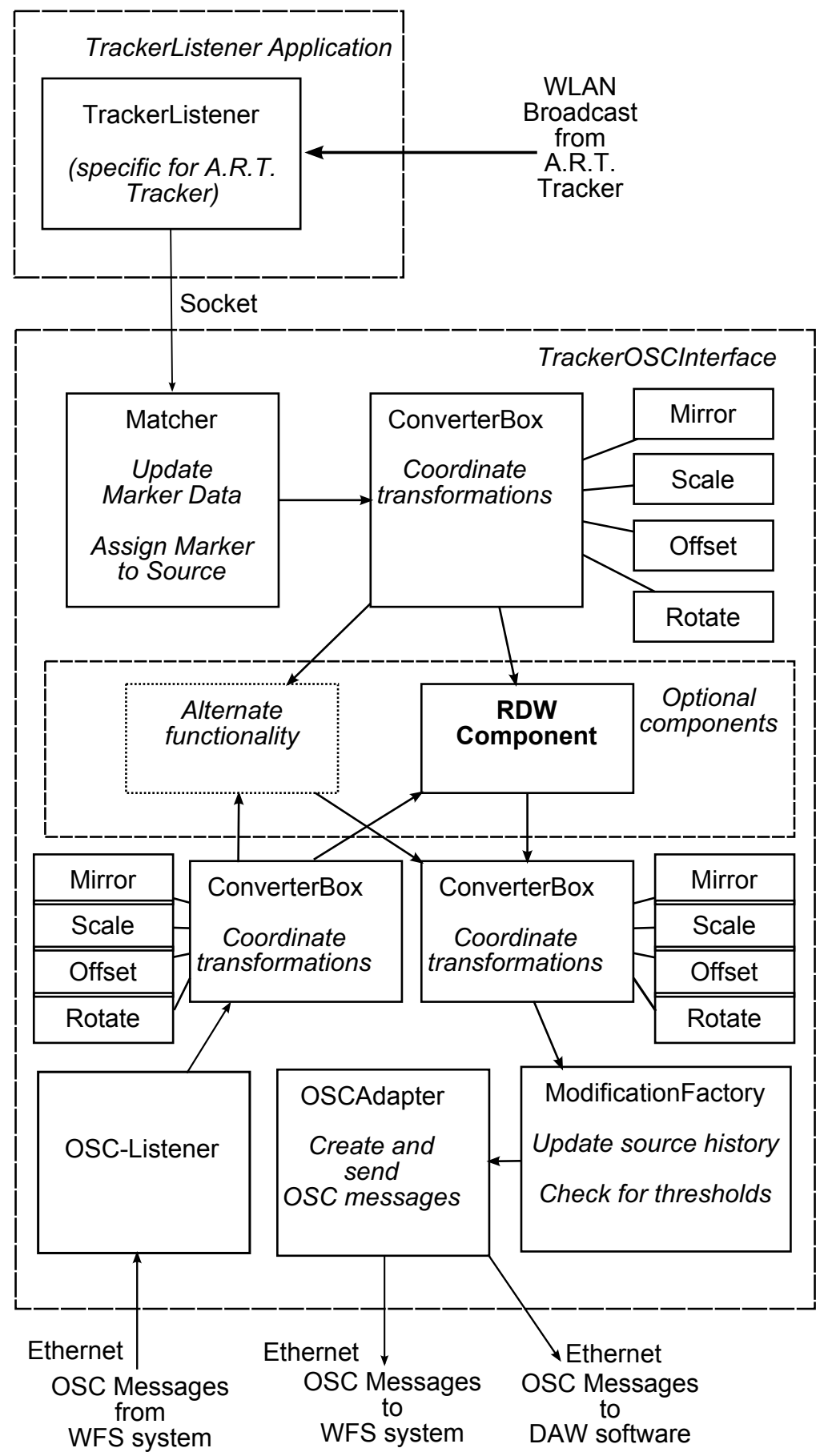

Figure 5.2.: Processing sequence of the Motion Tracker-Wave Field Synthesis-Connector (MoWeC). 


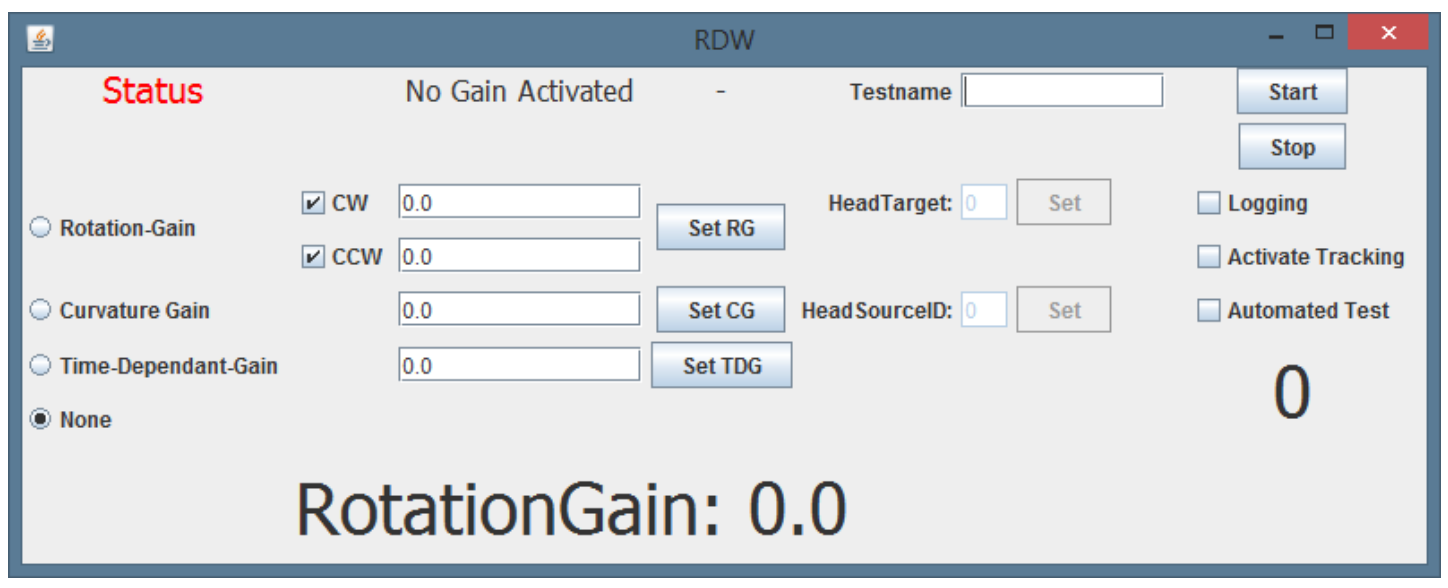

Figure 5.3.: The GUI for the $R D W$ component.

\subsection{Redirected Walking Component}

As mentioned before, the $R D W$ component is designed as an optional component for the $M o W e C$, as is illustrated in figure 5.2. As such, it is provided with tracking data from the tracking system and WFS data from the WFS system, both in form of MoWeC sources.

The tracking data from the head mounted target of the test subject provides the $R D W$ component with position and orientation data for the head of the test subject. According to the currently defined gain, a rotation value is calculated as described in section 7.2.3. This rotation value will be used to calculate new coordinates and orientations for all other $\mathrm{MoWeC}$ sources, to give the impression that the whole scene is rotating around the test subject. The MoWeC delivers these changes to the WFS system and the DAW in form of OSC messages.

The $R D W$ component has its own GUI, which is shown in figure 5.3. Starting this, the $M o W e C$ 's $G U I$ is bypassed and a number of settings are predefined, which otherwise would have to be set manually. Processing can be started and stopped and on the left hand side, different gains can be set manually. At the bottom, the activated gain is shown. On the right hand side, logging and tracking can be (de-)activated, as well as the automated test started. At the top, the automated tests can be named for logging purposes. For the experiment at hand, manual (de-)activation of gains is not necessary, however. By starting the automated test, gains will be chosen randomly and applied automatically. 


\section{Choice of Gains to be Tested}

This chapter explains how the gains for the experiment at hand were selected.

In (Steinicke et al., 2009) Steinicke et al. explain five different types of gains to manipulate users' movements within a redirected walking application: the translation gain, rotation gain, curvature gain, displacement gain and time-dependent gain. Time-dependent gains, however, have to be subdivided into time-dependent rotation gains and time-dependent translation gains. Gains generally map physical movements to VE motions, while time-dependent gains map depleted time to VE motion.

Translation gains, as a means to scale translations and described in Steinicke et al. (2009), were not considered for these experiments for long, since the acoustic perception of distance of humans is much more difficult than visual perception (Zahorik et al., 2005). It is presumed that vast manipulations can be made with translation gains, but that these will most likely not be perceived as a different self-motion. Much rather, the initial distance to the object might be perceived differently, and such give the impression of false dimensions of the VE. This might be interesting for acoustic $R D W$ applications in general but is not considered as one of the main methods. More interesting might be a version of the translation gain, which translates i. e. physical forward movements into virtual displacements orthogonal to the walking direction, which would have an effect close to the the one of the curvature gain. To keep the amount of tests for each test person in check, and because of the close relatedness to the curvature gain, it would not become part of this study either.

The displacement gain specifies physical rotations to virtual translations, which might be useful in some situations, but will most likely not become a significant part in most redirected walking application, due to high detection of the displacement or in conclusion small manipulation potential.

Time-dependent translation gains have been neglected due to the the reasons stated above. Time-dependent rotation gains have been considered and were part of a pilot study, but were discarded, because no test subject reacted to the manipulation as intended. Instead, after a short while everyone just reported, that the VE had rotated or some specific virtual sound source moved. 
Curvature gains and even more so rotation gains seem to be the most promising and raised, next to translation gains, the most attention so far Steinicke et al. (2008b,c,d,f); Bruder et al. (2009); Steinicke et al. (2009); Serafin et al. (2013). The self-conducted pilot studies also showed great potential for acoustic tests with the WFS system and therefore these two types became the center of this research.

Figure 2.5a illustrates an example of a bended path by the appliance of a curvature gain. The user perceives the virtual path as straight, while she really walks on a curved path. Figure $2.5 \mathrm{~b}$ illustrates an example of a scaled rotation by the appliance of an rotation gain. The user perceives the rotation as $180^{\circ}$, but really only rotates by $90^{\circ}$. 


\section{Experiment Design}

This chapter will explain the prepared experiment, starting with the requirements, followed by a description of the test procedure, and closing with the choice of gain values and different test groups. The design of the experiment has already been presented on the 12th Sound and Music Computing Conference in Maynooth, Ireland (Nogalski and Fohl, 2015).

\subsection{Requirements}

Some basic requirements had to be met during the development of the experiment, to enable success.

- Ambient noise

- to mask laboratory noises...

- and to help perceive the external-motion as self-motion.

- Sounds, that stimulate the test persons to turn on the spot.

- Sounds, that stimulate the test persons to walk.

\subsubsection{Ambient Noise}

The ambient noise has two main purposes. The first purpose is to mask laboratory noises, and the second purpose is to give more acoustic cues for orientation and essentially to make the $R D W$ manipulations more plausible.

The majority of experiments referred to in section 2 (Related Work) used headphones to play back the acoustic aspects of the IVE. In (Steinicke et al., 2008a) for example, headphones were used to play back some kind of ambient city noise "such that an orientation by means of auditory feedback in the real world was not possible" and in Neth et al. (2012) noise-canceling headphones playing white noise were used to mask real ambient noise. For $R D W$ by WFS noise-canceling headphones are out of the question, since they would, of course, cancel out the acoustic cues for the techniques as well. Actually, any kind of headphones were 
considered disturbing and unnecessary. Instead four linear sound sources were used to play back the ambient sounds through the WFS system. Because of the randomized sequence of the successive tests, which is explained later in this section, the path of the test subjects within the consistent $V E$ can not be foreseen. Test subjects could therefore close in on these virtual sound sources during their tasks. If they were point sound sources, the test subjects would notice the close proximity, and if they got too close, they could disturb the experiment, as they are not meant to be located within the focus of the test subjects. Linear sound sources however merely define a direction for the sound, which does not change when test subjects get close to them, and therefore proximity does not matter.

The four linear sound sources were oriented towards the four cardinal directions. Two opposite sound sources were mapped to the same, but phase shifted, monotonous city noise with some cars driving by, but little distinctive aspects ${ }^{1}$. Their main function is to mask the laboratory noises. One of the other sources got the sound of a playground ${ }^{2}$, and the opposite one of a small pedestrian zone ${ }^{3}$. These are mainly meant to give some secondary orientation.

\subsubsection{Sounds to Turn}

To stimulate the test subjects to turn, the sound of a small constantly barking $\operatorname{dog}^{4}$ was chosen and mapped to the turn-to target. Any sound would have sufficed, but intuitively people would turn and look towards a barking dog, and it appears plausible that this dog may change its position, such giving more freedom in designing the experiment while staying somewhat plausible.

\subsubsection{Sounds to Walk}

As a sound to stimulate the test persons to walk somewhere, the sound of an ice cream truck ${ }^{5}$ was chosen and mapped to the go-to target. Again, any sound would have sufficed, but in addition to the dog, this seemed like a plausible choice.

\footnotetext{
${ }^{1}$ https://www.freesound.org/people/balou82/sounds/184356/

${ }^{2}$ https://www.freesound.org/people/music.boy/sounds/119121/

${ }^{3}$ https://www.freesound.org/people/mario1298/sounds/155346/

${ }^{4}$ https://www.freesound.org/people/felix.blume/sounds/199261/ (This sound was modified to provide more regularity)

${ }^{5}$ https://www.freesound.org/people/ericstrausser/sounds/106238/ (This sound was cut, at the beginning and the end of the tune)
} 


\subsection{Automated Test Sequence}

To meet these requirements, an automation was designed, which leads the test subjects through all the tests of their experiment. This section starts with the conditions the automation will have to meet, and finally describes the sequence of the automation.

\subsubsection{Conditions}

One fundamental goal of the experiment design was, to conduct all tests for a test subject in one piece without breaks in presences. At no time during the experiment, test subjects should use other cues but aural, vestibular or proprioceptive. Some tests, however, have certain demands towards the starting position and/or orientation of the test subject. A curvature gain test, for an easy example, can not start with the test subject facing a wall. For these tests, prior to defining the individual starting positions and orientation, a likely path of the test subject will have to be predicted. While rotation gains could theoretically be tested in any position and angle, some aspects led to a closer definition of the starting conditions as well. The height of virtual sound sources within this WFS system is not position preservative. While auditors move through the plane, the perceived elevation of the virtual sound sources is dependent on the proximity to the relevant speakers. A virtual sound source outside of the physical WFS area will always be "behind" one of the speakers (see figure 7.1a). The speaker can be seen as the angle point of a seesaw with the aural perception system of an auditor on one side, and the virtual sound source on the other side, as is illustrated in figure 7.1. While the slope of this seesaw inand decreases with the proximity of the auditor to the relevant speaker, the perceived elevation of the virtual sound source also in- and decreases (see figure 7.1). The slope also changes, when the virtual sound source moves behind other speakers which are closer to or further away from the auditor. Since this effect can presumably give cues about the movement of virtual sound sources, rotation gain tests are defined to always take place in the center of the physical WFS area, where the variation of the distance between auditor and speaker, as well as between virtual sound source and speaker is least.

\subsubsection{Path Prediction}

For curvature gain tests, it is crucial to predict the path, the test subject is likely to take during the test. Even though the ideal path, given a starting position, starting orientation and curvature gain value, can be calculated, it is highly unlikely that the test subject will take this ideal path, considering that the $R D W$ technique is based on the principle of leading users to believe, that they strayed from the path. Amongst others, aspects like the precision of detecting the 

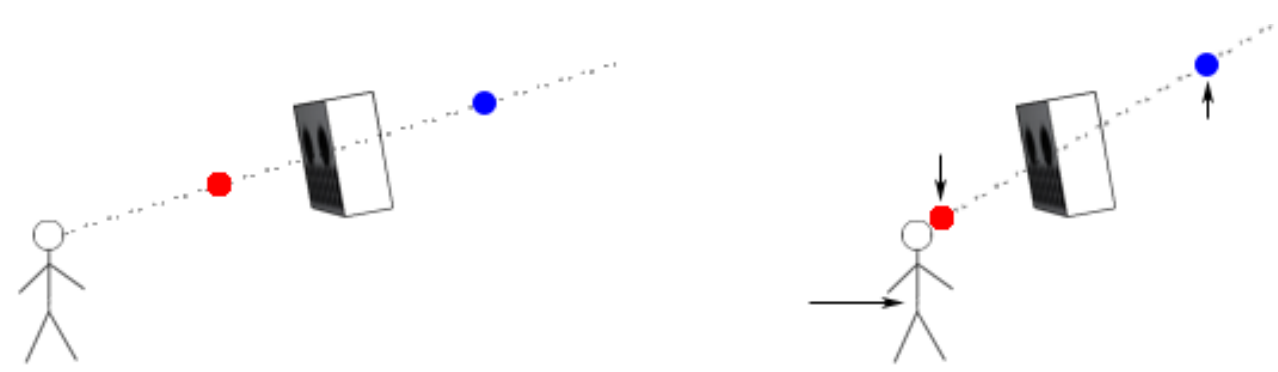

(a) Virtual sound sources are located on a straight(b) As the auditor is moving, the virtual sound defined by head and speaker. sources seem to change their height.

Figure 7.1.: The height of virtual sound sources is dependent on the relative position of the auditor to the relevant speakers. While the auditor moves closer to the speaker, the red virtual sound source will stay between the head and the speaker and the blue virtual sound source behind the speaker, and such changing their heights for this auditor.

direction of the targeted virtual sound source and the responsiveness to the manipulations will influence the path, the test subject is going to take. Minor manipulations will be calculated in a rapid succession, which are always dependent on the current position of the test subject and thus, even the ideal path changes with every stray from the previous ideal path.

Even though the exact path, which the test subject is going to take, can not be foreseen, an estimation can be made. The value of the curvature gain determines, in which direction the test subject will be redirected. Assumed the test subject is oriented towards the target before the test starts, it is highly unlikely that she will veer into the opposite direction by an significant amount. So the path ahead and towards the direction of the manipulation should be free. These tests will therefore start in corners of the room with an orientation towards the adjacent corner which opens the room towards the direction of the manipulation. Figure 7.2 illustrates the possible starting positions and orientations for curvature gains as well as for rotation gains.

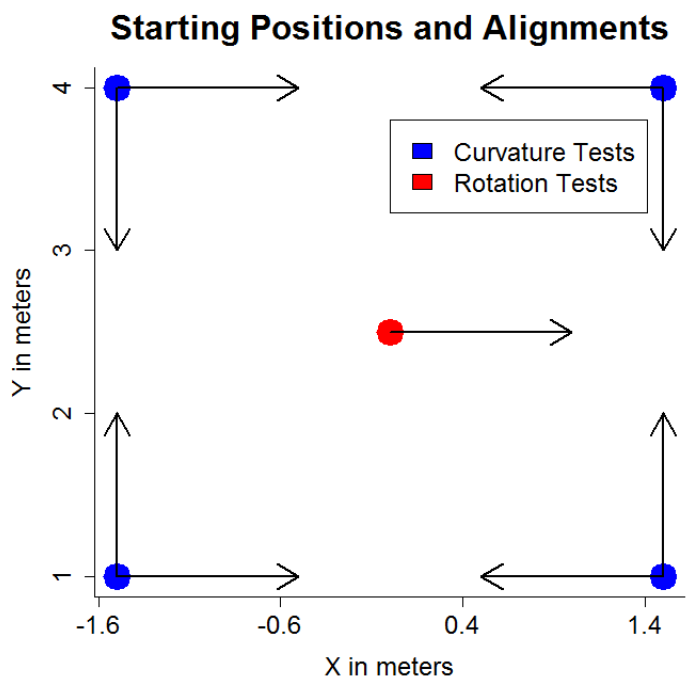

Figure 7.2.: All possible starting positions for curvature gain and rotation gain tests, with corresponding orientations. 


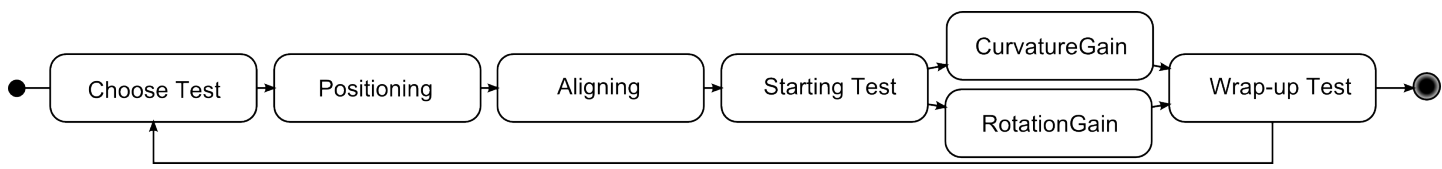

Figure 7.3.: During the automated tests, several states will be passed in a loop. At the start of each loop, a test is chosen randomly. Next the test person will be directed to the starting position of the test and then be aligned into the starting direction. Depending on the current test, the test setup will be set and then the actual test will be started and executed until the test subject fulfilled the conditions. To wrap each test up, further logging and deactivation of sounds is necessary before determining, whether any more tests are left.

\subsubsection{Automation}

The automation is to ensure that all test will be executed in a random order seamlessly and without any further instructions or interferences during the experiment. The state diagram in figure 7.3 illustrates the individual steps of the automation.

Choose Test - The automation follows an iterative pattern and for each test, the first step is always the randomized selection of a test that had not been conducted yet.

Positioning - Depending on the type of test and the current position of the test subject, a starting position for the test is determined. Rotation gain tests will always be performed in the middle of the physical WFS area, while curvature gain tests will start in the closest corner. Any corner would do, but for the convenience of the test subject, the closed corner is chosen. See figure 7.2 for all possible starting positions. The go-to target is then placed on the starting position for the test, and its sound is activated. The sound will then keep running in a loop, until the test subject has reached a certain minimum proximity to the go-to target. Upon arrival within the target area around the go-to target, the sound is turned of, and positioning is complete.

Aligning - The starting alignment is also dependent on the type of test. Rotation gain tests always start with an alignment along the $\mathrm{x}$-axis, and curvature gain tests with an alignment that allows the test subject to follow the manipulation towards the center of the room. See figure 7.2 for all possible starting alignments in regard to the starting position. After the starting alignment has been identified, the turn-to target is placed on a position in that direction and its sound is activated. For curvature gains, the position will be the position of the corner in that direction. For rotation gains, the position will always be 10 meters from the rotation gain starting position along the $\mathrm{x}$-axis. The sound will then keep playing a loop, until a head orientation of the test subject had been captured which is within a certain deviation to the 
calculated orientation towards the turn-to target. Then the sound will stop playing and after a pause of two seconds, the aligning is complete. This pause is to ensure, that the test subject has settled into that direction, and to help distinguish between the sounds of the alignment and the following test.

Starting Tests - Before the manipulation can start, the corresponding virtual sound source has to be placed and activated. For curvature gain tests, the go-to target is placed at the position of the corner to which the test subject had just been oriented during the previous step. For rotation gain tests, the turn-to target is positioned 10 meters from the rotation gain starting position in the opposite direction from the previous alignment. The next step is highly dependent on the type of test.

Curvature gain - For a curvature gain test, each decrease in distance towards the go-to target is multiplied by the value of the curvature gain to calculate the corresponding rotation of all virtual sound sources around the test subject. This state will be active, until the test subject has reached a distance to the starting position which is higher than the distance from the starting position to the go-to target. In this case, the test subject passed the go-to target, is most likely very close to it, and would now turn around to locate the exact position. Since this is not so much subject of the experiment and to speed up the process, the conditions are considered met.

Rotation gain - When a rotation gain test had been selected, all head rotations of the test subject will be multiplied with the value of the rotation gain and result in a corresponding rotation of all virtual sound sources around the test subject. This state will be active, until the difference between the orientation of the test subjects head and the angle towards the turn-to target is below a certain threshold. Then the manipulation will stop, but the sound will keep playing for another second, so the target does not just vanish during the rotation.

Wrap-up Test - The last step for each iteration is the wrap up. During the wrap up, the turn-to target and go-to target are muted, the test will be marked as done and removed from the to-do list, and the to-do list is checked for more elements. If more tests need to be done, the iteration starts from the beginning and otherwise, the experiment will end.

\subsection{Gain Values}

The choice of the values of the gains to be tested is based on related work by Steinicke et al. Steinicke et al. (2009) and modified by a couple of self-conducted tests, to get good limits and distribution in between. Ideally, the greatest manipulations should be noticed by all test subjects and the smallest by none, to cover the whole range. 
For rotation gains the range is $-60 \%<=g_{R}<=+60 \%$ with an increment of $5 \%$. For curvature gains the range is $-1.0<=g_{C}<=+1.0$, with $g_{C}:=\frac{1}{r}$ and $r$ being the radius of the circle that is defined by the curve, also with an increment of 0.05 . Tests with a value of 0 are included four times each.

\subsection{Test Groups}

The test subjects were originally divided into three groups, but due to findings while conducting the experiment, a fourth group was formed.

Group 1 (Naive): These test subjects did not know that they were participating in a $R D W$ experiment. Instead they were told that the focus of the experiment was on the position preservation of focused sound sources. They were asked to report whenever they felt that a virtual sound source would move, even though it might just be a subjective perception.

Group 2 (Aware): These test subjects were aware that they were participating in a $R D W$ experiment. They were informed about the technique and how the system would try to manipulate their path due to rotations of the IVE. They did not know however, in which situations, how strong, and how often these manipulations would occur. They were instructed to report any notice of the VE rotating around them. Presumably, this knowledge would influence the sensitivity to detect the manipulations (Steinicke et al., 2010). This is considered the most relevant group. Prospective users of an IVE, which is using auditory $R D W$ techniques, will most likely be aware that these manipulations may occur.

Group 3 (Expert): These test subjects knew exactly, how the automation of the experiment works. They knew the proportion of movements with manipulations to movements without manipulations, and most likely knew in which situations a manipulation was likely to occur. All of them had witnessed an experiment as a bystander, and had an extensive discussion about the experiment beforehand. One of these test subjects even was the developer of this experiment. This group is considered the most challenging with presumably the highest detection rate. Prospective users of an redirected walking application will most likely have less information about the occurring manipulations, since these manipulations will only occur when the system identifies a need to do so. With presumably little to no knowledge of one's position and orientation within the physical WFS area after a couple of manipulations, these situations will probably not be foreseeable by other users. 
Group 4 (two-alternative forced-choice (2AFC)): These test subjects had the same knowledge of the experiment as group 2 (Aware), but had to give a feedback after each applied gain in the manner of a $2 A F C$ task. After each gain they had to reply either with "right" or "left", indicating into what direction (as perceived from their own position and orientation) they perceived a rotation of the $V E$, respectively a movement of the object. In situations in which the test subjects were unsure they had to go by intuition or guess. Through this method more information about the detection thresholds should be obtained, especially in areas of small gains. Further explanation will follow while discussing the results.

The division into these groups will allow evaluation of the sensitivity to the manipulations regarding perceptibility in dependency of the knowledge of the system and situation.

"In cases, in which users do not actively pay attention to possible manipulations, the thresholds shall be significantly higher." (Steinicke et al., 2010) 


\section{Results}

This chapter starts with an overview of all test subjects and performed trials of the experiment. The presentation of the results will start with the merged results of all groups, and then differentiates them by the four groups. Sections 8.8 - 8.11 will illuminate some specific aspects.

Across all test subjects a total of 2777 trials (1689 curvature gains and 1088 rotation gains) have been conducted successfully. 31 additional trials could not be completed or evaluated due to technical problems. The mean total duration for each subject was 27.5 minutes. Table 8.1 lists the quantity for all gain-value combinations, and figure 8.1 illustrates an exemplary curvature gain trial with $g_{C}(0.4)$.

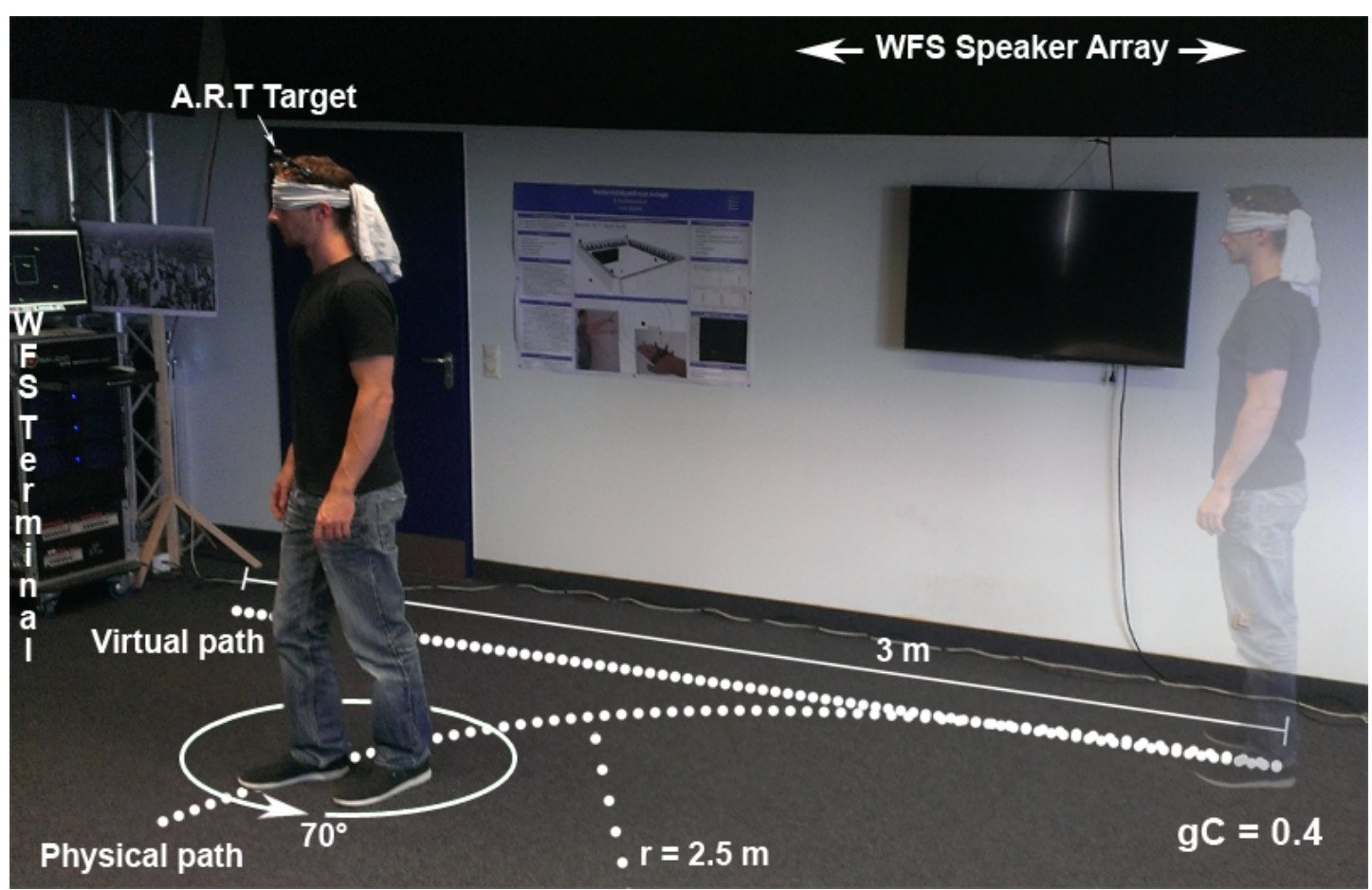

Figure 8.1.: An example of a curvature gain trial with $g_{C}(0.4)$. The length of both paths is 3 meters. The ideal physical path is a segment of a circle with a radius of 2.5 meters, which changes the direction by $70^{\circ}$ after 3 meters. 


\begin{tabular}{lll|lll} 
Type & Value & Count & Type & Value & Count \\
\hline$g_{C}$ & 0.00 & 152 & $g_{R}$ & -0.6 & 39 \\
$g_{C}$ & 0.05 & 76 & $g_{R}$ & -0.55 & 39 \\
$g_{C}$ & 0.10 & 78 & $g_{R}$ & -0.5 & 39 \\
$g_{C}$ & 0.15 & 78 & $g_{R}$ & -0.45 & 39 \\
$g_{C}$ & 0.20 & 76 & $g_{R}$ & -0.4 & 39 \\
$g_{C}$ & 0.25 & 78 & $g_{R}$ & -0.35 & 39 \\
$g_{C}$ & 0.30 & 77 & $g_{R}$ & -0.3 & 39 \\
$g_{C}$ & 0.35 & 77 & $g_{R}$ & -0.25 & 39 \\
$g_{C}$ & 0.40 & 76 & $g_{R}$ & -0.2 & 39 \\
$g_{C}$ & 0.45 & 76 & $g_{R}$ & -0.15 & 39 \\
$g_{C}$ & 0.50 & 78 & $g_{R}$ & -0.1 & 39 \\
$g_{C}$ & 0.55 & 77 & $g_{R}$ & -0.05 & 39 \\
$g_{C}$ & 0.60 & 76 & $g_{R}$ & 0 & 156 \\
$g_{C}$ & 0.65 & 78 & $g_{R}$ & 0.05 & 39 \\
$g_{C}$ & 0.70 & 77 & $g_{R}$ & 0.1 & 38 \\
$g_{C}$ & 0.75 & 75 & $g_{R}$ & 0.15 & 39 \\
$g_{C}$ & 0.80 & 76 & $g_{R}$ & 0.2 & 39 \\
$g_{C}$ & 0.85 & 77 & $g_{R}$ & 0.25 & 39 \\
$g_{C}$ & 0.90 & 77 & $g_{R}$ & 0.3 & 38 \\
$g_{C}$ & 0.95 & 78 & $g_{R}$ & 0.35 & 38 \\
$g_{C}$ & 1.00 & 76 & $g_{R}$ & 0.4 & 38 \\
& & & $g_{R}$ & 0.45 & 39 \\
& & & $g_{R}$ & 0.5 & 39 \\
& & & $g_{R}$ & 0.55 & 39 \\
& & & $g_{R}$ & 0.6 & 39
\end{tabular}

Table 8.1.: Test count by type and value 


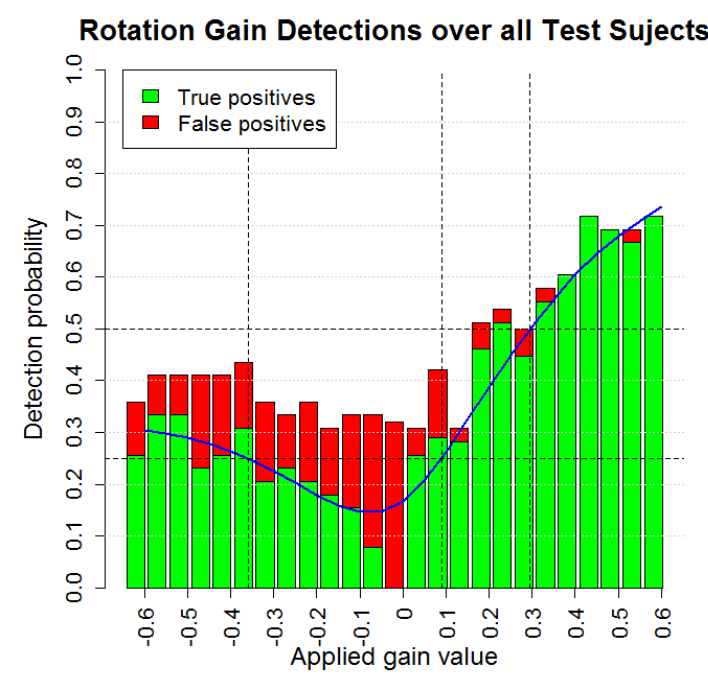

(a) Rotation gains

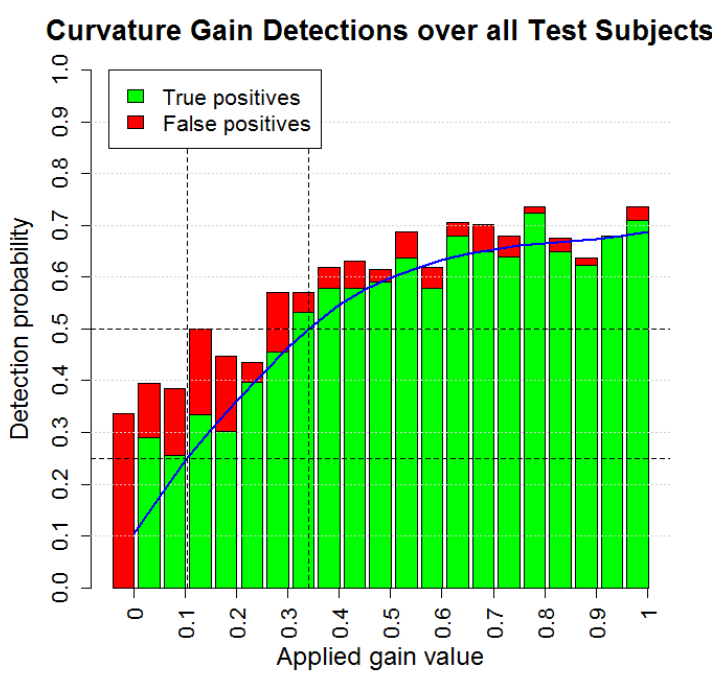

(b) Curvature gains

Figure 8.2.: Gain detections over all test subjects. The applied values on the $\mathrm{x}$ axis and the observed detection rate on the y axis.

\subsection{Participants}

A number of 39 (10 female and 29 male) test subjects participated in the experiment. The ages ranged from 21 to 66 . The averange age was 29.44. 20 test subjects were students of technical, applied or general computer sciences, 7 were students of other course of studies. Amongst the others were one professor of computer science, a pensioner, clerks and more.

\subsection{Groups}

The test subjects have been divided into four groups as described in section 7.4. 9 subjects are located in group 1 (Naive), 17 in group 2 (Aware), 4 in group 3 (Expert) and 9 in group 4 (2AFC). One test subject of group 1 (Naive) and one of group 2 (Aware) were afterwards trained to also become test subjects of group 3 (Expert), and are considered as two test subjects each.

Figure 8.2 illustrates the observed detection rates over all test subjects, but, as will be presented during the following sections, the detection rates differ significantly between the different groups, so these combined results over all test subjects may not be the most representative for future applications.

The green "true positive" bars of the plots within figure 8.2 and all following similar plots represent the ratio of correctly recognized and stated manipulations compared to the total 
number of manipulations for that specific gain-value-combination. The red "false positive" bars represent wrongly recognized and stated manipulations. So, as illustrated in figure 8.2a for all test subjects, $20 \%$ recognized the manipulation with rotation gain(-0.6) in the correct direction, $10 \%$ recognized a manipulation in the wrong direction, and $65 \%$ did not state to have noticed any manipulation.

Consider that a $10 \%$ additional rotation is not equal to a resulting total rotation of $110 \%$, because another additional $10 \%$ are also applied to the formerly added $10 \%$, which results in another $1 \%$ additional rotation and so on.

The rotation gain detection threshold of $25 \%$ was reached at 0.1 ( $10 \%$ additional rotation) for positive gains which up-scales a users virtual rotation $R_{\text {virtual }}$ of $180^{\circ}$ by $\sim 11 \%$ to a physical rotation $R_{\text {physical }}$ of $200^{\circ}$, and at -0.40 (-40\% less rotation) for negative gains which down-scale a users rotation of $180^{\circ}$ by $\sim 29 \%$ to $\sim 129^{\circ}$.

The rotation gain detection threshold of $50 \%$ was reached at 0.3 for positive gains which up-scales the rotation by $\sim 42 \%$ to $\sim 257^{\circ}$, and the highest detection rate for negative gains was barely $35 \%$ at a value of -0.50 which down-scales the rotation by $33.3 \%$ to $120^{\circ}$. Equation 8.1 illustrates the calculations.

$$
\begin{aligned}
R_{\text {physical }} & =\frac{R_{\text {virtual }}}{1-g_{R}} \\
R_{\text {physical }} & =\frac{180}{1-0.1} \quad=200^{\circ} \\
R_{\text {physical }} & =\frac{180}{1-(-0.4)} \approx 129^{\circ} \\
R_{\text {physical }} & =\frac{180}{1-0.3} \approx 257^{\circ} \\
R_{\text {physical }} & =\frac{180}{1-(-0.50)}=120^{\circ}
\end{aligned}
$$

The curvature gain detection threshold of $25 \%$ was reached at a value of 0.1 which would amount to a circle with a radius of 10 meters.

$$
\begin{aligned}
r & =\frac{1}{g_{C}} \\
r & =\frac{1}{0.1}=10 \mathrm{~m}
\end{aligned}
$$




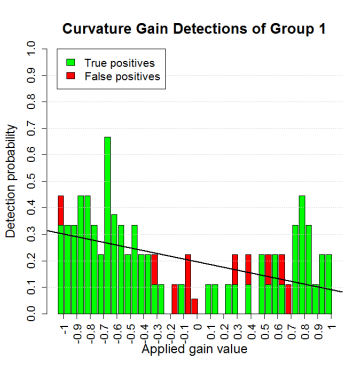

(a) Group 1 (Naive)

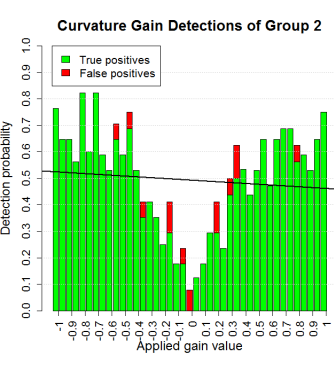

(b) Group 2 (Aware)

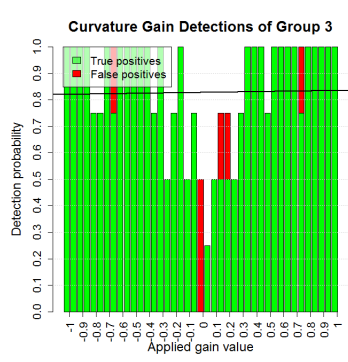

(c) Group 3 (Expert)

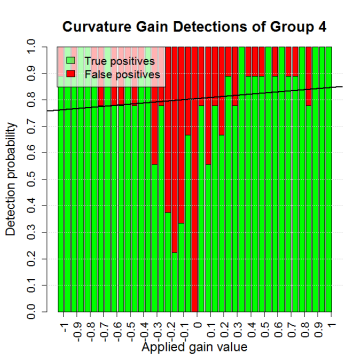

(d) Group 4 (2AFC)

Figure 8.3.: Curvature gain detections with respect to the gain being positive or negative (bending the path to the left or right) divided by groups. No consistent trend can be observed while comparing the detections of positive and negative gains.

The curvature gain detection threshold of $50 \%$ was reached at a value of 0.35 which would amount to a circle with a radius of 2.86 meters.

$$
\begin{aligned}
& r=\frac{1}{g_{C}} \\
& r=\frac{1}{0.35} \approx 2.86 \mathrm{~m}
\end{aligned}
$$

Figure 8.4 shows the curvature gain detections of all test subjects with respect to the gain being positive or negative. A positive gain would bend the physical path to the left, and a negative gain to the right. Even though the detection rate of negative curvature gains that bend the path to the right was slightly higher, a paired Wilcoxon signed rank test delivers a probability of $18.93 \%$ that the hypothesis of a difference between positive and negative gains is wrong. Thus, the tendency of the best-fit-line in figure 8.4 can not be considered statistically significant, and the hypothesis must be rejected. Figure 8.3 illustrates that the tendencies also differ between the groups. Therefore, for further evaluation

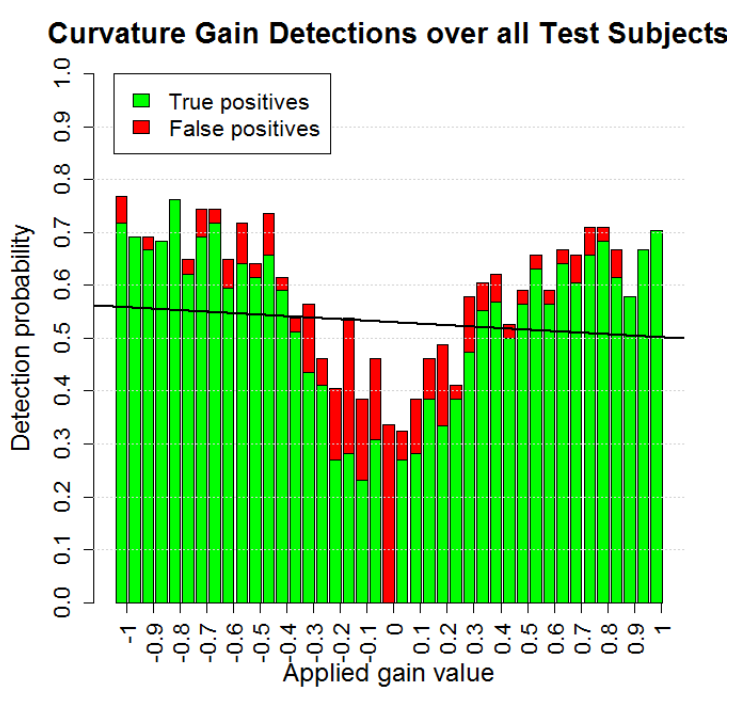

Figure 8.4.: Curvature gain detections over all test subjects with respect to the gain being positive or negative. 


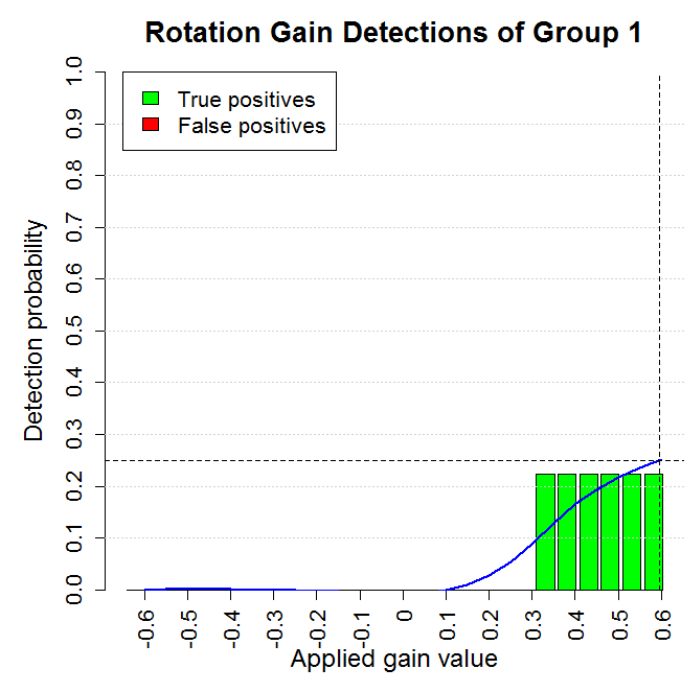

(a) Rotation gains

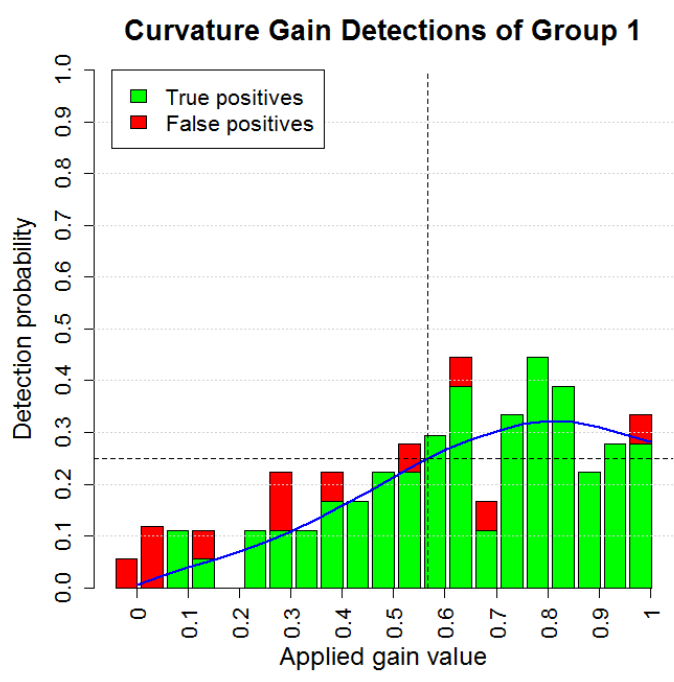

(b) Curvature gains

Figure 8.5.: Gain detections of group 1 (Naive). The applied values on the $\mathrm{x}$ axis and the observed detections on the y axis.

only the absolute gain values will be used. Thus, positive and negative gains are merged to receive more tests per value.

\subsection{Group 1 (Naive)}

Figure 8.5 illustrates the observed detections of the 9 test subjects of group 1 (Naive).

For rotation gains, no detections were observed for negative values at all, and within the positive sector coincidentally two detections were observed for all values between 0.3 and 0.6. The resulting maximum detection threshold is $22 \%$ which up-scales the rotation by $\sim 43 \%$ $150 \%$ to $257^{\circ}(0.3)-450^{\circ}(0.6)$. One subject detected one of all of these detected manipulations (which results in a 50\% detection rate for these 6 gains for this test subject), while the other six detections were spread across three more subjects.

$$
\begin{aligned}
& R_{\text {physical }}=\frac{R_{\text {virtual }}}{1-g_{R}} \\
& R_{\text {physical }}=\frac{180}{1-0.3} \approx 257^{\circ} \\
& R_{\text {physical }}=\frac{180}{1-0.6}=450^{\circ}
\end{aligned}
$$




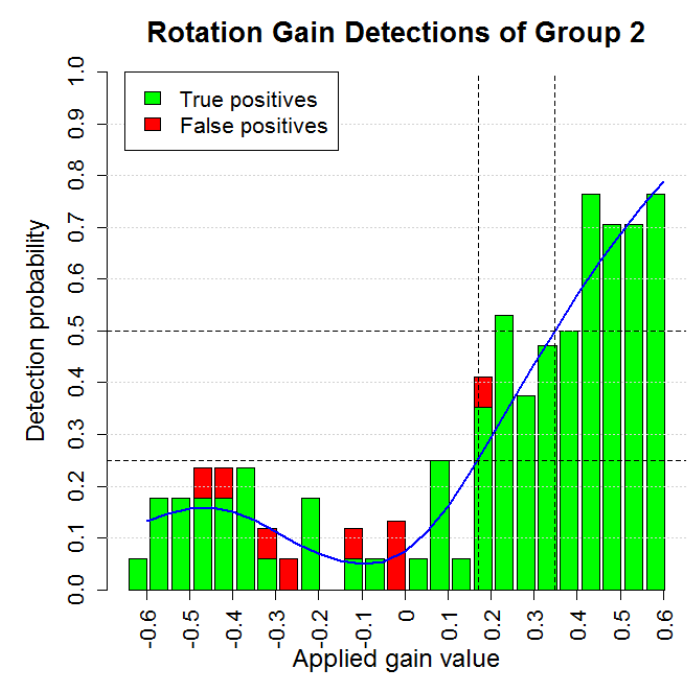

(a) Rotation gains

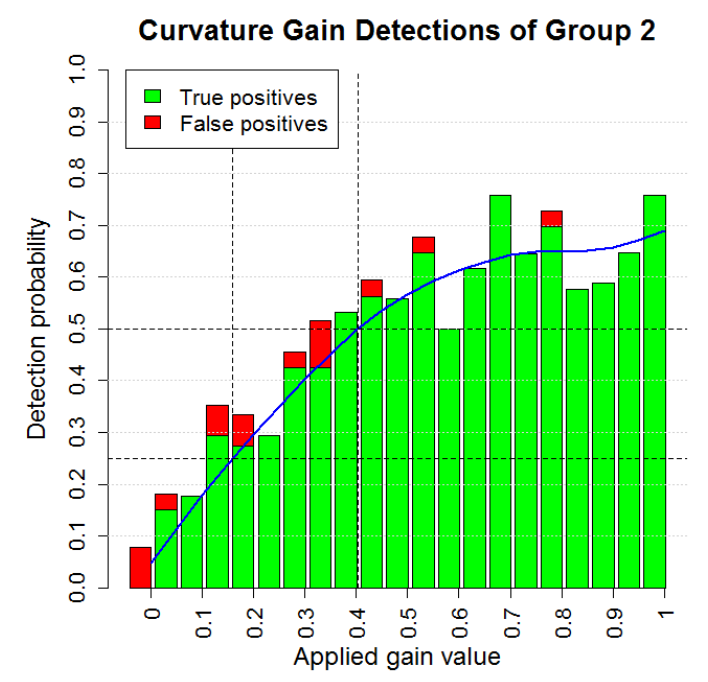

(b) Curvature gains

Figure 8.6.: Gain detections of group 2 (Aware). The applied values on the $\mathrm{x}$ axis and the observed detections on the y axis.

For curvature gains, the detection threshold of $25 \%$ was reached at a gain of 0.55 which would amount to a circle with a radius of 1.82 meters. The highest detection rate of $33 \%$ was observed at gains of 0.8 which would amount to a circle with a radius of 1.25 meters.

$$
\begin{aligned}
r & =\frac{1}{g_{C}} \\
r & =\frac{1}{0.55}=1 . \overline{81} \mathrm{~m} \\
r & =\frac{1}{0.8}=1.25 \mathrm{~m}
\end{aligned}
$$

\subsection{Group 2 (Aware)}

Figure 8.6 illustrates the observed detections of the 17 test subjects of group 2 (Aware).

For rotation gains, the peak within the negative value range of $15 \%$ had been reached at -0.45 which down-scales the rotation by $31.1 \%$ to $124^{\circ}$. Within the positive sector the $25 \%$ detection threshold had been reached at 0.155 which up-scales the rotation by $18.3 \%$ to $213^{\circ}$. The $50 \%$ 
detection threshold had been reached at 0.35 which up-scales the rotation by $54 \%$ to $277^{\circ}$, and after which the detection rate kept rising steep.

$$
\begin{aligned}
& R_{\text {physical }}=\frac{R_{\text {virtual }}}{1-g_{R}} \\
& R_{\text {physical }}=\frac{180}{1-(-0.45} \approx 124^{\circ} \\
& R_{\text {physical }}=\frac{180}{1-0.155} \approx 213^{\circ} \\
& R_{\text {physical }}=\frac{180}{1-0.35} \approx 277^{\circ}
\end{aligned}
$$

For curvature gains, the detection threshold of $25 \%$ was reached at a gain of 0.15 which would amount to a circle with a radius of 6.6 meters. The $50 \%$ detection threshold was reached at a gain of 0.4 which would amount to a circle with a radius of 2.5 meters. The highest detection rate was observed at gains of 0.7 and 1.0 which would amount to a circle with a radius of 1.43 meters, and after which no more significant changes could be observed.

$$
\begin{aligned}
r & =\frac{1}{g_{C}} \\
r & =\frac{1}{0.15}=6 . \overline{6} \mathrm{~m} \\
r & =\frac{1}{0.4}=2.5 \mathrm{~m} \\
r & =\frac{1}{0.7} \approx 1.43 \mathrm{~m}
\end{aligned}
$$

\subsection{Group 3 (Expert)}

Figure 8.7 illustrates the observed detections of the 4 test subjects of group 3 (Expert). The group of expert test subjects consisted of the designer of the experiment and author of this thesis, the professor to oversee the thesis and two students, who did already take part in this experiment within group 1 (Naive) or group 2 (Aware), had an extensive discussion about the process and sequence of the experiment, and witnessed the experiment, while another test subject was tested. These circumstances led to a good understanding of the process of the experiment and in most situations the test subjects could estimate the state of the experiment and therefore predict probabilities for manipulations. However, the exact applied gains could not be predicted due to the randomized selection.

To exploit most of the available tracking area and physical WFS area, before each curvature gain test subjects were aligned along one of the boundaries of the tracking area, as described 


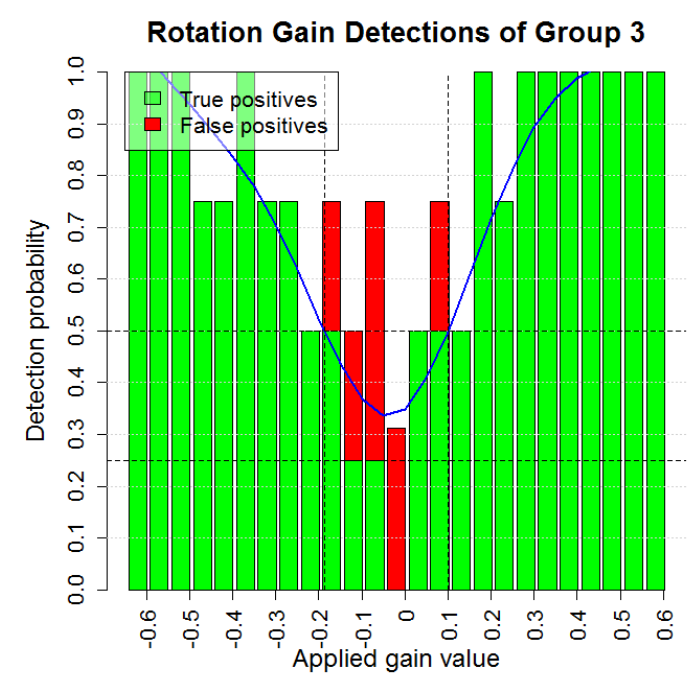

(a) Rotation gains

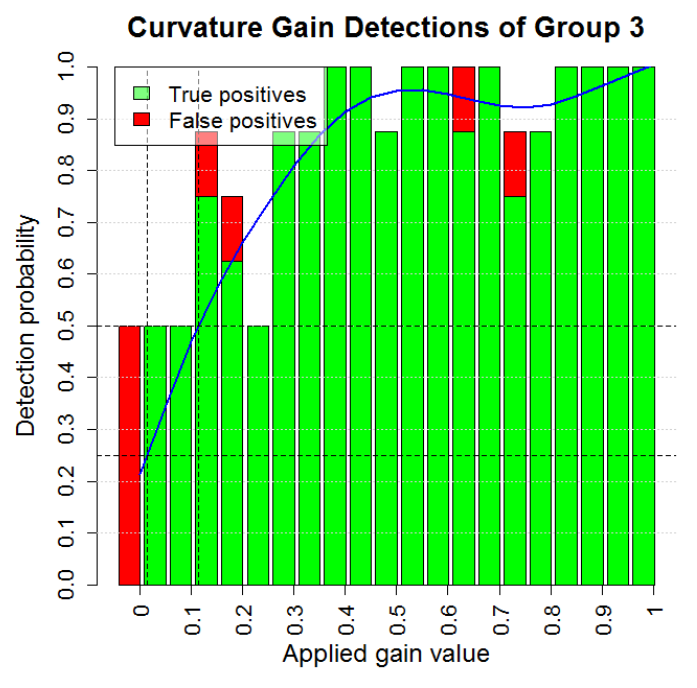

(b) Curvature gains

Figure 8.7.: Gain detections of group 3 (Expert). The applied values on the $\mathrm{x}$ axis and the observed detections on the y axis.

in section 7.2.2. Due to the expertise of the test subjects in group 3 (Expert), they could track the state of the experiment to the extent that they could eliminate the probability for either a positive or negative gain for any applied curvature gain which left them with the probability of $\frac{20}{24}$ for a manipulation into the possible direction or $\frac{4}{24}$ for no manipulation. Some thought had been put into repeating the curvature gain part of the experiment for group 3 (Expert), but the necessary adjustments would have changed the experiment to an even less comparable state. So for this evaluation these results have to be regarded with special care.

For curvature gains, the detection rate started at $50 \%$, but the false positive rate of likewise $50 \%$ at the zero-gain and at gains of 0.15 and 0.2 may indicate that the manipulations could not be perceived reliably at values up to 0.25 which would amount to a circle with a radius of 4 meters.

$$
\begin{aligned}
r & =\frac{1}{g_{C}} \\
r & =\frac{1}{0.25}=4 \mathrm{~m}
\end{aligned}
$$

For rotation gains even the expert test subjects could not make a prediction about the value of the applied gain, except due to the distributions of values. Especially the knowledge of the small chance of a zero-gain of $\frac{4}{28}$ might have seduced the test subjects to perceive a manipulation 

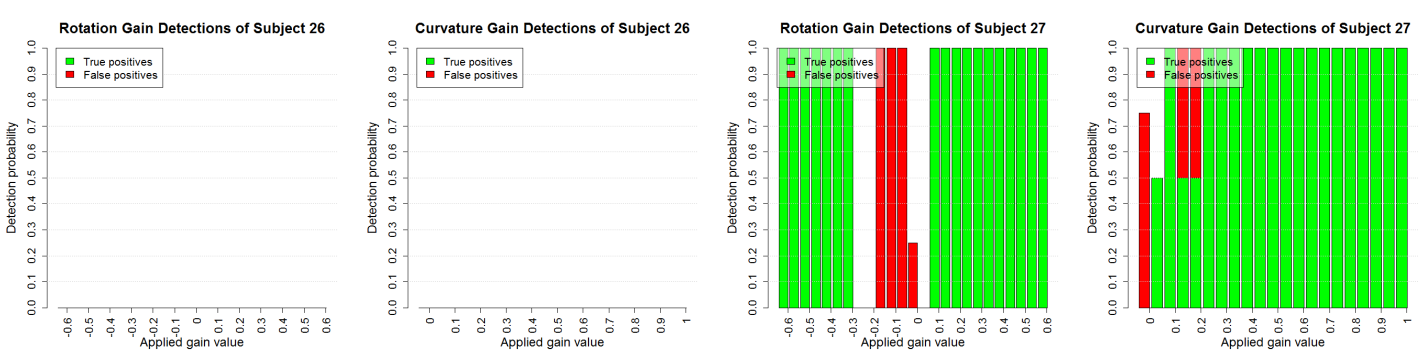

(a) Rotation gains subject 26/27. (Naive).

$$
\text { Group } 1
$$
ject 26/27. Group 1 (Naive). (b) Curvature gains sub- -(c) Rotation gains subject (d) 26/27. Group 3 (Expert). (d) Curvature gains subject 26/27. Group 3 (Expert).

Figure 8.8.: Gain detections of test subject 26/27. The same subject participated first in group 1 (Naive) as subject 26 and later in group 3 (Expert) as subject 27. The difference between figures $8.8 \mathrm{a} \& 8.8 \mathrm{~b}$ and $8.8 \mathrm{c} \& 8.8 \mathrm{~d}$ are results of only the increased knowledge of the experiment when participating in group 3 (Expert).

where otherwise they would have not. This knowledge likely explains the high false positive rate at gains between -0.15 and +0.1 which might indicate a higher portion of chance within the true positives within this range of gains as well. On this grounds, an unreliable detection rate between the values of -0.2 and +0.15 may be justified. This would indicate that the rotations of the expert group could have been up-scaled by $17.7 \%$ to $\sim 212^{\circ}$ and down-scaled by $16.6 \%$ to $150^{\circ}$.

$$
\begin{aligned}
& R_{\text {physical }}=\frac{R_{\text {virtual }}}{1-g_{R}} \\
& R_{\text {physical }}=\frac{180}{1-(-0.2)}=150^{\circ} \\
& R_{\text {physical }}=\frac{180}{1-0.15} \approx 212^{\circ}
\end{aligned}
$$

As mentioned above, two of the test subjects of group 4 (2AFC) had participated in the experiment before within another group. The figures 8.8 and 8.9 compare the results of both test subjects within the different groups. Please consider for these plots that each rotation gain had only been applied once and each curvature gain twice for each test subject except for the gain values of zero which had been applied four times. Test subject 26 and 27 are the same person. He participated in group 1 (Naive) first with the appropriate knowledge and did not state any detections, as is illustrated in the figures $8.8 \mathrm{a}$ and $8.8 \mathrm{~b}$. After he had been briefed for group 3 (Expert), he detected $82 \%$ correctly. Figure 8.9 illustrates the results of the test subject who participated in group 2 (Aware) under number 5 and then in group 3 (Expert) under 


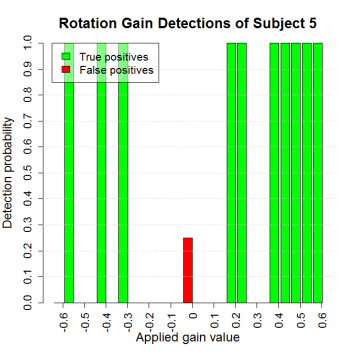

(a) Rotation gains subject (b) 5/6. Group 2 (Aware).
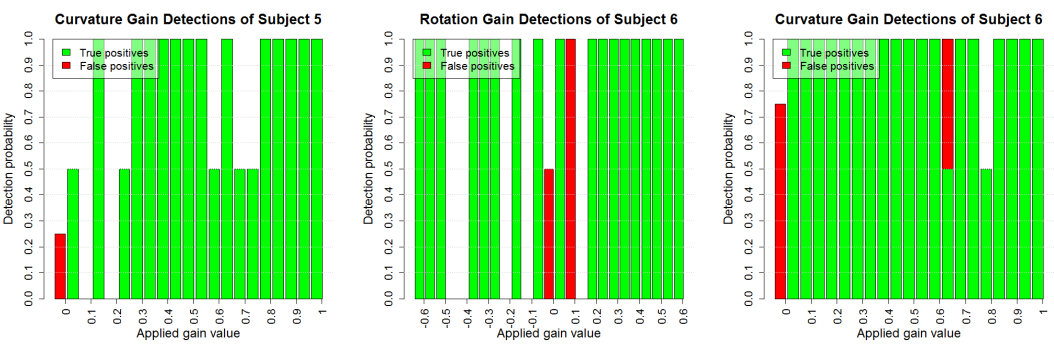

(b) Curvature gains subject 5/6. Group 2 (Aware). -(c) Rotation gains subject (d) Curvature gains sub5/6. Group 3 (Expert). ject 5/6. Group 3 (Expert).

Figure 8.9.: Gain detections of test subject 5/6. The same subject participated first in group 2 (Aware) as subject 5 and later in group 3 (Expert) as subject 6. The difference between figures $8.9 \mathrm{a} \& 8.9 \mathrm{~b}$ and $8.9 \mathrm{c} \& 8.9 \mathrm{~d}$ are results of only the increased knowledge of the experiment when participating in group 3 (Expert).

number 6. Even though he already had a high detection rate for group 2 (Aware), the detection rate raised significantly after the briefing as well.

\subsection{Group 4 (2AFC)}

Even before evaluating the results of group 1 (Naive) and group 2 (Aware), just by monitoring the experiments, many results were astonishing. During curvature gains, test subjects often took severely crooked and bend paths with obvious course corrections during a trial, but still did not give a feedback to indicate that they perceived a manipulation of the VE. All 26 test subjects of group 1 (Naive) and group 2 (Aware) showed this behaviour during multiple trials. For a lot of trials, it was highly unlikely that the test subjects perceived their paths as straight, as it should be when not giving a feedback. According to the experiment design, test subjects were supposed to walk directly towards the go-to target under the assumption that they would only have to walk a bend path, when the go-to target would be moving. In conclusion they should always perceive a manipulation, whenever they had to leave the straight path. Respectively the same behaviour was observed during rotation gains, where test subjects turned a full $360^{\circ}$, to face an object that originally was placed right behind them.

This behaviour could only be explained by a lack of confidence of the test subjects in their ability, to identify the direction of the object through acoustic cues. This speculation was strengthened by the feedback of verbal interviews with the test subjects after completion of all trials. A lot of subjects reported, that they felt, that they had problems finding the objects. 
Evaluation of trials with zero gain and the observation of the behaviour during phases of positioning or aligning in between trials showed that the precision was actually very good. Section 8.8 will give some examples of paths taken by test subject during the trials. Figure 8.16 illustrates the precision of a test subject during the four curvature gains(0). In most cases, the test subjects found the go-to target without any kind of or just minor path corrections.

To elude this problem of missing feedback due to the insecurity of the test subjects, this fourth test group was formed which was not intended when originally designing the experiment. The experiment was slightly altered, so all 9 test subjects of group $4(2 A F C)$ had to give a feedback whenever, after reaching a go-to target or facing a turn-to target, no new object would appear (which would be the case after the completion of a trial). The options stayed the same ("left" or "right"). For low gain values, where the manipulation would not be perceived, the proportion between "left" and "right" responses should even out close to 50\% "left" and 50\% "right". For higher gains, where test subjects would start to perceive the manipulation more reliable, the imbalance of the proportion should rise. By forcing the test subjects to give a feedback after each trial, the experiment should yield more information that was previously withheld.

The previous knowledge of the test subjects about the experiment was equal to that of group 2 (Aware). For comparison the $50 \%$ threshold of the results of group 4 (2AFC) is equivalent to the $0 \%$ threshold of the other groups and the $75 \%$ threshold of group $4(2 A F C)$ is equivalent to the $50 \%$ threshold of the other groups. Because the test subjects could only choose between the "right" or "left" feedback, they could not give a correct feedback for zero gains. For the evaluation "right" had been arbitrary chosen to be considered the right answer.

Figure 8.10 illustrates the observed detections of the 9 test subjects of group 4 (2AFC).

For rotation gains within the negative value range, the detection threshold of $25 \%$ had barely been reached at the maximum rotation gain value of -0.6 which down-scales the rotation by $37.5 \%$ to $112.5^{\circ}$. Within the positive sector the $25 \%$ detection threshold had been reached at 0.05 which up-scales the rotation by $\sim 5 \%$ to $\sim 190^{\circ}$. The $50 \%$ detection threshold had been reached at 0.15 which up-scales the rotation by $\sim 17.7 \%$ to $\sim 212^{\circ}$.

$$
\begin{aligned}
& R_{\text {physical }}=\frac{R_{\text {virtual }}}{1-g_{R}} \\
& R_{\text {physical }}=\frac{180}{1-(-0.6)}=112.5^{\circ} \\
& R_{\text {physical }}=\frac{180}{1-0.05} \approx 190^{\circ} \\
& R_{\text {physical }}=\frac{180}{1-0.15} \approx 212^{\circ}
\end{aligned}
$$




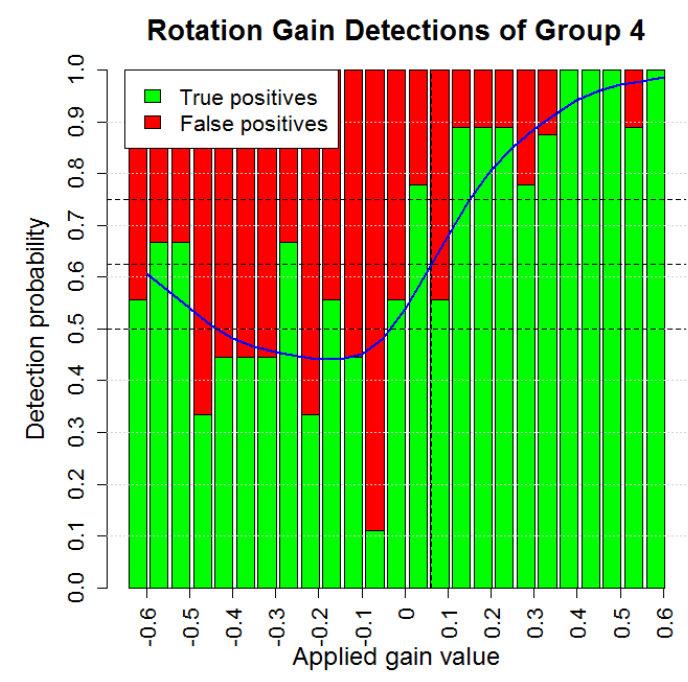

(a) Rotation gains

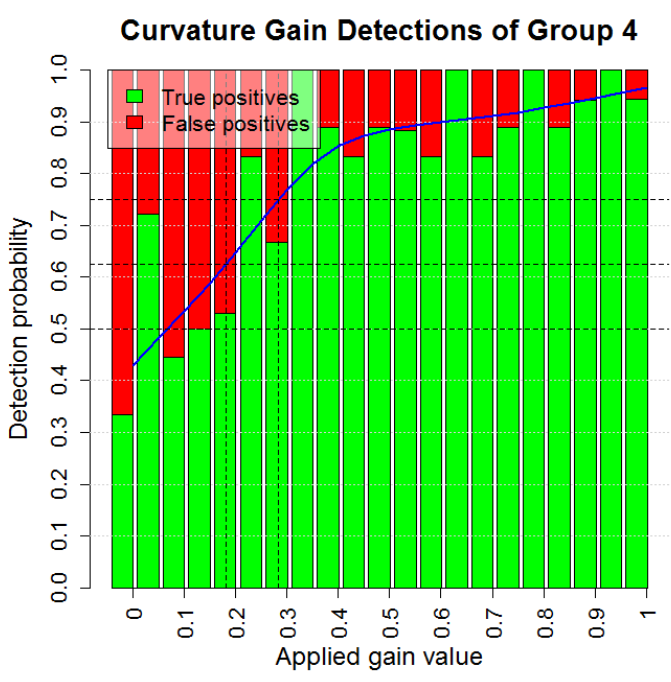

(b) Curvature gains

Figure 8.10.: Gain detections of group 4 (2AFC). The applied values on the $\mathrm{x}$ axis and the observed detections on the y axis.

For curvature gains, the detection threshold of $25 \%$ (62.5\% correct answers) was reached at a gain of 0.175 which would amount to a circle with a radius of 5.71 meters. The detection threshold of 50\% (75\% correct answers) was reached at at a gains of 0.275 which would amount to a circle with a radius of 3.64 meters. After a gain of approximately 0.4 the detection slope starts ascending less steep, but up until the highest gain values, some wrong feedbacks are within the results.

$$
\begin{aligned}
& r=\frac{1}{g_{C}} \\
& r=\frac{1}{0.175} \approx 5.71 \mathrm{~m} \\
& r=\frac{1}{0.275}=3 . \overline{63} \mathrm{~m}
\end{aligned}
$$

The figures $8.11 \mathrm{a}$ and $8.11 \mathrm{~b}$ show the paths of the trials of the highest two curvature gains of group 4 ( $2 A F C$ ), for which a wrong feedback was given. At the begin of the trial illustrated in figure 8.11a, the test subject, despite the aligning sequence, did not start walking directly towards the go-to target, but probably coincidently walked into the direction where the go-to target would be moving. Through the manipulation, around half way through the trial, the go-to target moved into the direction, where the test subject was moving. Until then, the go-to target moved considerable, but the test subject did not have to adjust his direction. Towards 
Path of subject $\mathrm{nr} .30$ and gain value -1

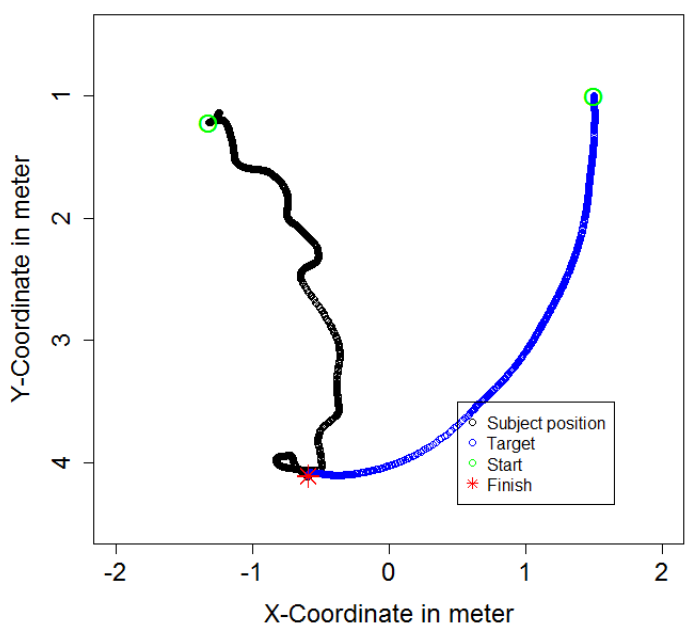

Path of subject $\mathrm{nr} .30$ and gain value -0.9

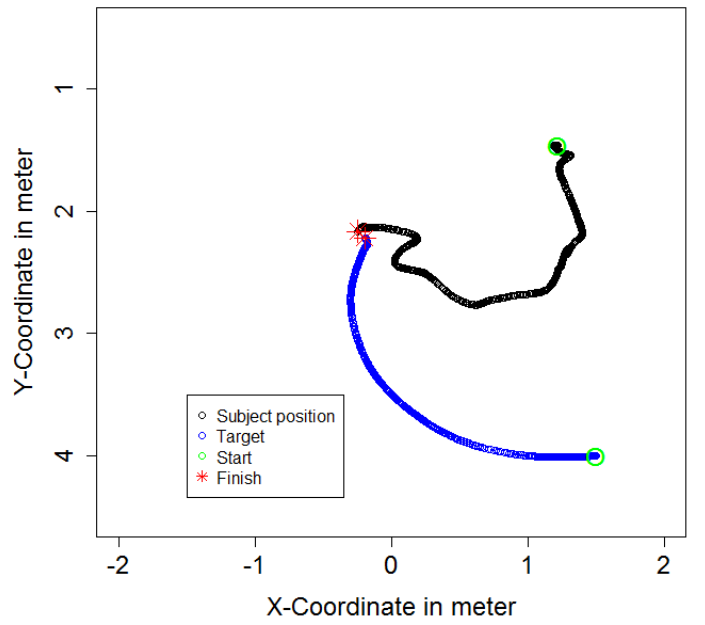

(b) Incorrect detection due to oversteering at the

(a) Incorrect detection due to initial deviation from(b) Incorrect detectio
the ideal path and oversteering at the end. end of the trial.

Figure 8.11.: Two paths during trials with very high curvature gains which nonetheless resulted in a wrong (or no) detection.

the end of the trial at around position $(-0.5,3)$, the test subject started to adjust his path towards his right, to keep walking towards the go-to target which had now passed him. In the very end he even oversteered, and as a result passed the go-to target on the right side which probably led to the wrong impression that the object had moved to the left. Figure $8.11 \mathrm{~b}$ illustrates a path, where the test subject did a major adjustment around the middle of the trial, but obviously did not hold the moving $V E$ responsible for this necessity. Instead he oversteered during another distinctive adjustment towards the end of the trial and again passed the object on the right side, and therefore perceived the object as moving left rather than right. In both cases it is also possible, of course, that the test subject did not perceive any movement at all, but due to the $2 A F C$ aspect of the experiment, just gave the wrong answer by chance. 


\subsection{Comparison of Detection Thresholds}

This section summarizes the results of the different groups and previous work for easy comparison.

\subsubsection{Rotation Gain}

Table 8.2 summarizes the results of the rotation gain trials of all groups. The columns represent the groups, and the rows represent the detection thresholds for up-scaling (positive) and down-scaling (negative) gains. The respective percentage shows, by how much the test group could be manipulated for the given threshold.

\begin{tabular}{l|rrrrr|c} 
Threshold Group & Merged & $\mathrm{Gr}^{1}$ & $\mathrm{Gr}^{2}$ & $\mathrm{Gr}^{3}$ & $\mathrm{Gr}^{4}$ & Serafin et al. $^{5}$ \\
\hline 25\% up-scale & $\sim 11 \%$ & $\sim 43 \%$ & $18.3 \%$ & - & $\sim 5.0 \%$ & - \\
$25 \%$ down-scale & $\sim 29 \%$ & - & - & - & $37.5 \%$ & - \\
$50 \%$ up-scale & $\sim 42 \%$ & - & $54 \%$ & $17.7 \%$ & $\sim 17.7 \%$ & $20 \%$ \\
$50 \%$ down-scale & - & - & - & $16.6 \%$ & - & $18 \%^{6}$
\end{tabular}

Table 8.2.: Results of the rotation gain detection thresholds by detection threshold vertically, and groups horizontally. The values indicate, by how much a rotation could be upor down-scaled.

\subsubsection{Curvature Gain}

Table 8.3 summarizes the results of the curvature gain trials of all groups. The columns represent the groups, and the rows represent the retection thresholds. The respective value in meters represents the radius of the circle that the curve is indicating.

\begin{tabular}{l|lrrrr|c} 
Threshold $^{\text {Group }}$ & Merged & $\mathrm{Gr}^{1}$ & $\mathrm{Gr}^{2}$ & $\mathrm{Gr}^{3}$ & $\mathrm{Gr}^{4}$ & Serafin et al. ${ }^{5,7}$ \\
\hline $25 \%$ & $10 \mathrm{~m}$ & $1.81 \mathrm{~m}$ & $6.6 \mathrm{~m}$ & $\infty \mathrm{m}$ & $5.71 \mathrm{~m}$ & - \\
$50 \%$ & $2.86 \mathrm{~m}$ & - & $2.5 \mathrm{~m}$ & $4 \mathrm{~m}$ & $3.64 \mathrm{~m}$ & $11.45 \mathrm{~m}^{8} / 16.37 \mathrm{~m}^{9}$
\end{tabular}

Table 8.3.: Results of the curvature gain detection thresholds represented as the radius of the circle defined by the curve. At $\infty$ all gains were detected.

\footnotetext{
${ }^{1}$ Group 1 (Naive)

${ }^{2}$ Group 2 (Aware)

${ }^{3}$ Group 3 (Expert)

${ }^{4}$ Group $4(2 A F C)$

${ }^{5}$ Results published in (Serafin et al., 2013).

${ }^{6} \mathrm{~S}$. Serafin confirmed in personal correspondence that a mistake slipped into the textual representation of the results. $18 \%$ is correct (as the corresponding plot and the value 0.82 suggest) instead of $+12 \%$.
} 
Path of subject $\mathrm{nr} .37$ and gain value 0.2

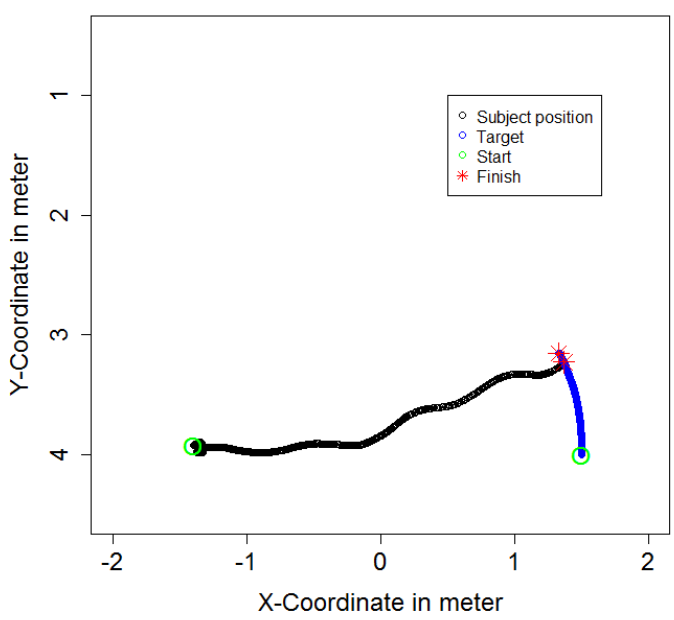

(a) Curvature gain(0.2) within group 4 (2AFC) (b) $(2 A F C)$ not perceived.
Path of subject $\mathrm{nr} .37$ and gain value $\mathbf{0 . 3}$

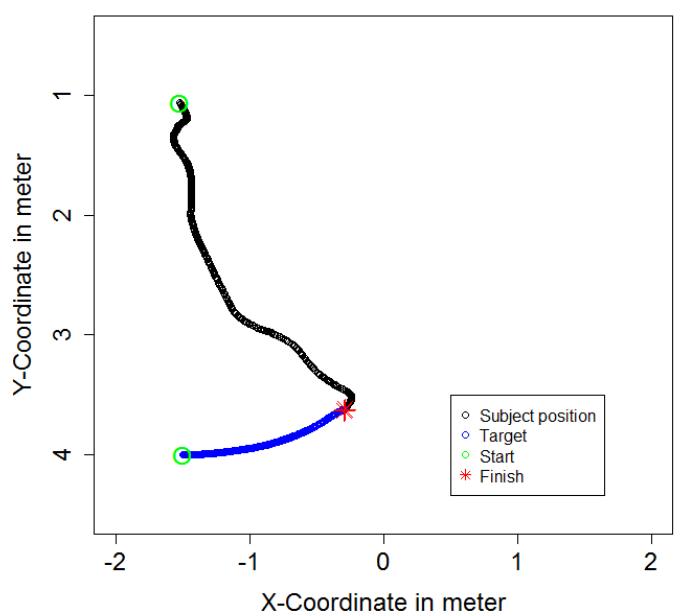

(b) Curvature gain(0.3) within group 4 (2AFC) $(2 A F C)$ not perceived.

Figure 8.12.: Two paths, during which the test subject walked a comparatively even curve and did not perceive the manipulation. The test subject gave the wrong answer within the $2 A F C$ trial, so the manipulation was not detected.

\subsection{Exemplary Paths}

To provide a clearer picture on how the curvature gains affected movements of the test subjects in detail, and how the huge discrepancies between the detection thresholds of the groups could come about, this section and the figures 8.12 to $8.15 \mathrm{a}$ will give some examples of paths that test subjects took during their trials.

The black circles within the figures (due to the density of different positions, the path mostly appears as a bold black line) represent the positions of the test subject during the trial which amount to the path the test subject took. The blue circles represent the path that the go-to target took during the trial. Green circles mark the start position of the test subject and go-to target. Red crosses mark the final positions of test subject and go-to target.

The figures $8.12 \mathrm{a}$ and $8.12 \mathrm{~b}$ show examples of paths a test subject within group 4 (2AFC) took during the trials. In these cases, the test subject adapted very nice and evenly to the

\footnotetext{
${ }^{7} \mathrm{~S}$. Serafin confirmed in personal correspondence that a mistake slipped into the textual representation of the results. +10 is correct (as the corresponding plot illustrates) instead of +30 .

${ }^{8}$ Result of the highest $g_{C}$ value of -25

${ }^{9}$ Result of the mean $g_{C}$ value of negative and positive gains. Since the gains in that experiment were equally distributed between positive and negative values and for better comparison with the results of the work presented here, these are merged into an absolute $g_{C}$ value of 17.5.
} 


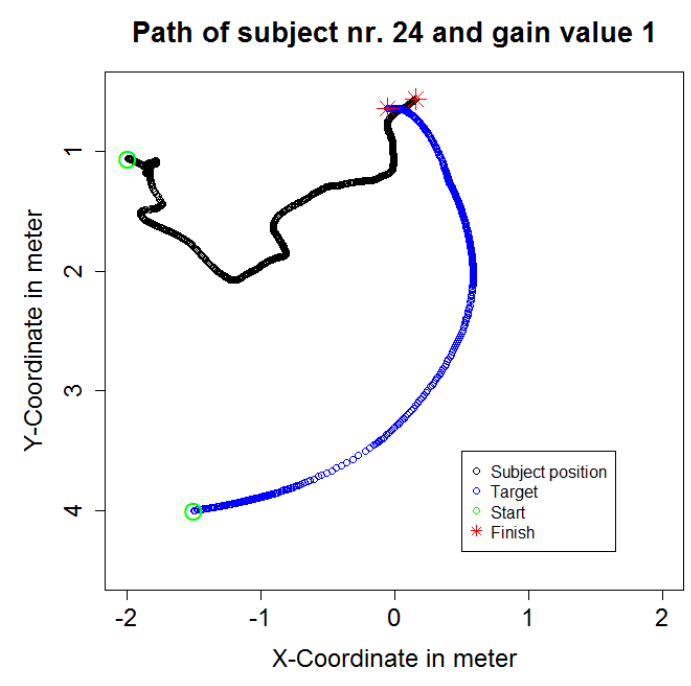

(a) Curvature gain(1.0) not perceived.

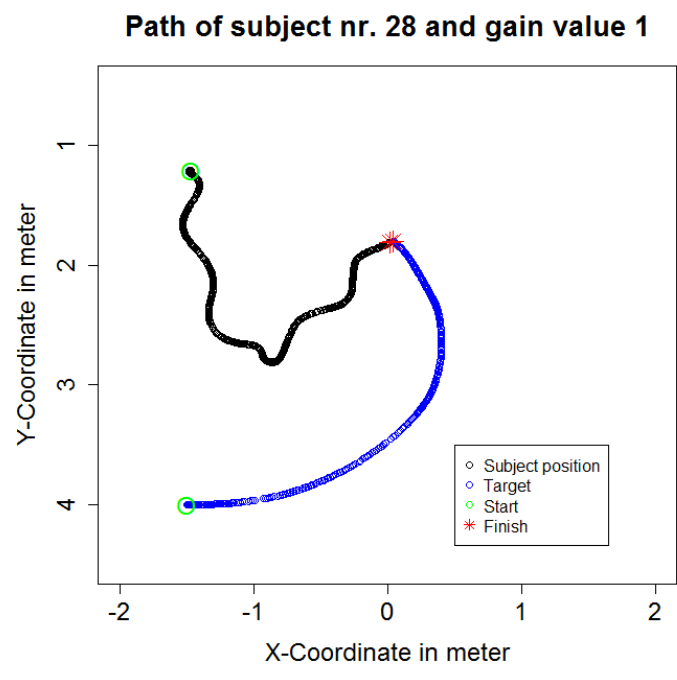

(b) Curvature gain(1.0) not perceived.

Figure 8.13.: Two paths, in which the test subjects did not perceive a manipulation, even though the manipulation was severe (curvature gain $(1.0)=>$ a radius of 1 meter) and the subjects were aware of their crooked path.

manipulation. Most of the irregularities could most likely be traced back simply to the veering of the test subject due to the common variability in stepping direction. (Kallie et al., 2007) illuminates the veering especially for blind walkers. The trial of figure $8.12 \mathrm{a}$ had a curvature gain of 0.2 (radius of $5 \mathrm{~m}$ ) and the trial of figure $8.12 \mathrm{~b}$ a curvature gain of 0.3 (radius of 3.3). Neither manipulations were perceived.

The figures 8.13a and 8.13b show paths of subjects in group 1 (Naive) and group 2 (Aware) with the highest curvature gain of 1.0 , of the trials (radius of $1 \mathrm{~m}$ ). Obviously the manipulation to the $V E$ had been severe. The go-to target moved by multiple meters and the test subjects had to perform extensive path corrections. While the test subjects were aware of their crooked paths, they did not perceive the manipulations, but neither did they succumb to the illusion that their paths were straight. This can be explained for the case, in which the test subjects came to terms with misjudging the initial direction of the go-to target and only detect their mistake at a later stage of the trial. The figures $8.14 \mathrm{a}$ and $8.14 \mathrm{~b}$ show speculatively manipulated images of the paths of figure 8.13, as they might have been perceived.

The figures 8.15a and 8.15b show exceptionally extraordinary paths. Two subjects showed an unexpected behaviour. On multiple occasions, when they were on the verge of detecting a manipulation, they stopped and started walking backwards with the intention of retracing 
Path of subject nr. 24 and gain value 1

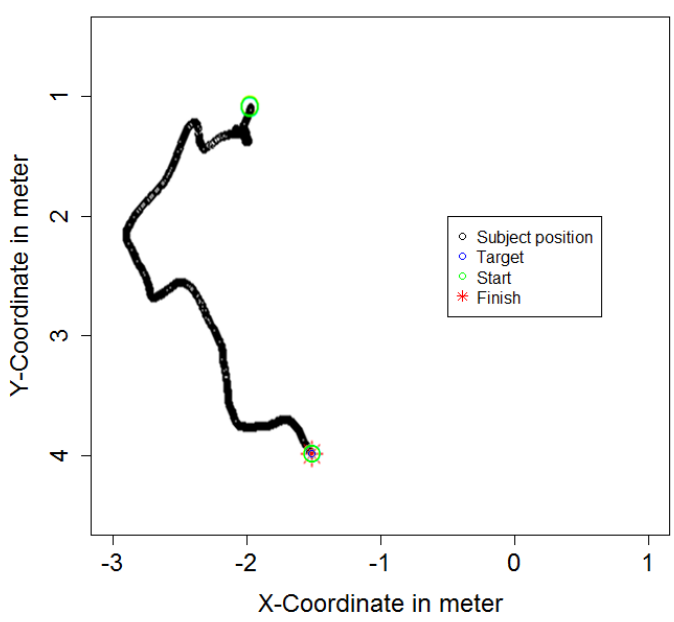

(a) Path of figure 8.13a modified, as it might have (b) Path of figure 8.13b modified, as it might have been perceived.
Path of subject nr. 28 and gain value 1

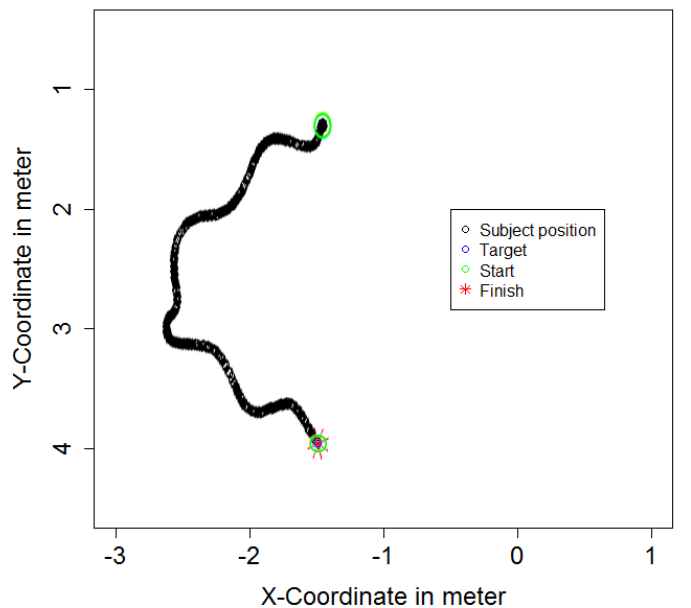

been perceived.

Figure 8.14.: The two paths of figure 8.13 speculatively modified, as they might have been perceived.

their steps back to the starting position, to start that particular trial from the beginning, and to pay extra attention on the second or even third try. This behaviour was totally unexpected and presented a flaw within the experiment design, as well as stunning results.

As described in section 7.2, only the part of the movement of the test subjects that was directed towards the go-to target was used to calculate the rotation of the VE. Walking backwards towards the starting position had therefore no effect on the VE. Instead, the go-to target maintained the position it had been moved to during the first part, when the test subject was walking towards it. As a result, the test subject maintained his orientation towards the new position while walking backwards, and even though he retraced his steps back close to the starting position, when he wanted to restart the trial, he had already been severely manipulated into facing into another direction. Peculiar and indeed very stunning to observe is the fact that in the case of figure $8.15 \mathrm{~b}$ the test subject was in the end satisfied with the consistency of the $V E$, even though he had turned by $180^{\circ}$ at the end of the trial and the curvature gain was with 1.0 the highest in the experiment.

The flaw within the experiment design, which had been exposed by this behaviour, was that test subjects would still be able to move the go-to target outside of the tracking area despite the careful positioning of the test subjects according to chosen trials and path predictions, because by walking forth and back repeatedly, the go-to target could potentially move entire circles 
Path of subject $\mathrm{nr} .22$ and gain value -0.65

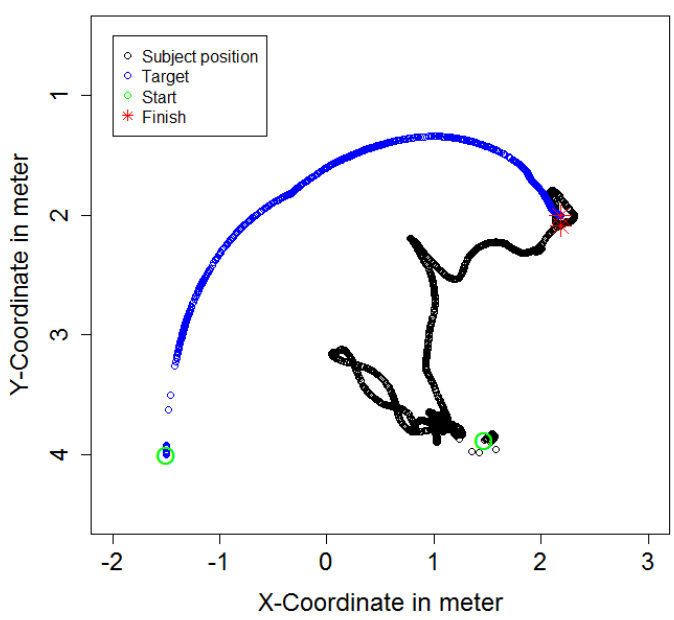

(a) Curvature gain(-0.65) perceived. Gaps within the path of subject and go-to target originate from interrupts of the tracking system.
Path of subject nr. 22 and gain value 1

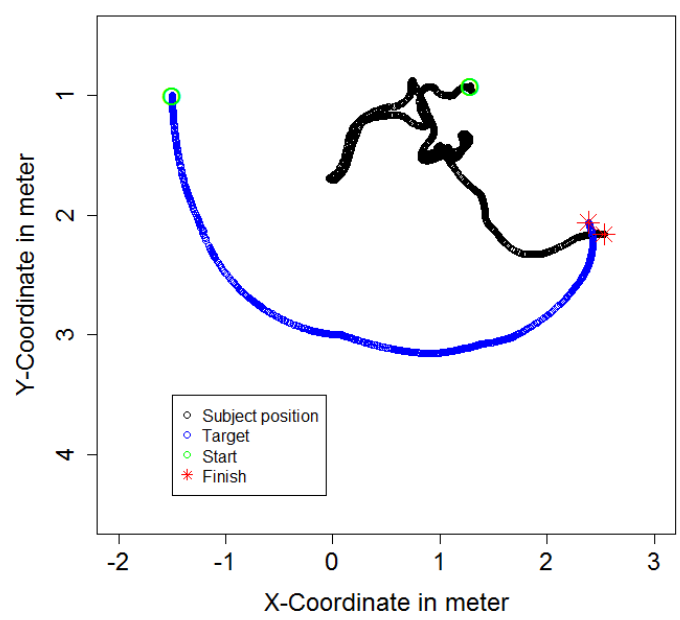

(b) Curvature gain(1.0) not perceived.

Figure 8.15.: Two paths, in which the test subject tried to retrace his steps to the starting position to restart the trial. Note the rescaled coordinate system compared to other path plots.

around the test subject. In both illustrated cases the test subject left the intended test area and came dangerously close to the physical walls of the laboratory. The area of the coordinate system shown in figures $8.15 \mathrm{a}$ and $8.15 \mathrm{~b}$ had to be enlarged, to show the final positions of the test subject.

The irregularities within the curvature of the go-to target's path (very clearly in figure $8.15 \mathrm{~b}$ at position $(0.2,3.1))$ arose, because the test subject changed his position, while walking backwards, without changing the position of the go-to target, and upon "restarting" the trial, the go-to target started turning around this new position and therefore changing its course.

The four paths illustrated in figure 8.16 are all the curvature gains with value 0.0 that the test subject experienced. It is plain to see that the general sense of direction towards the acoustic object was quite precise, and that the test subject had no problem in finding the go-to target. Only one path shows a distinctive inaccuracy followed by a course correction. 
Path of subject nr. 28 and gain value 0

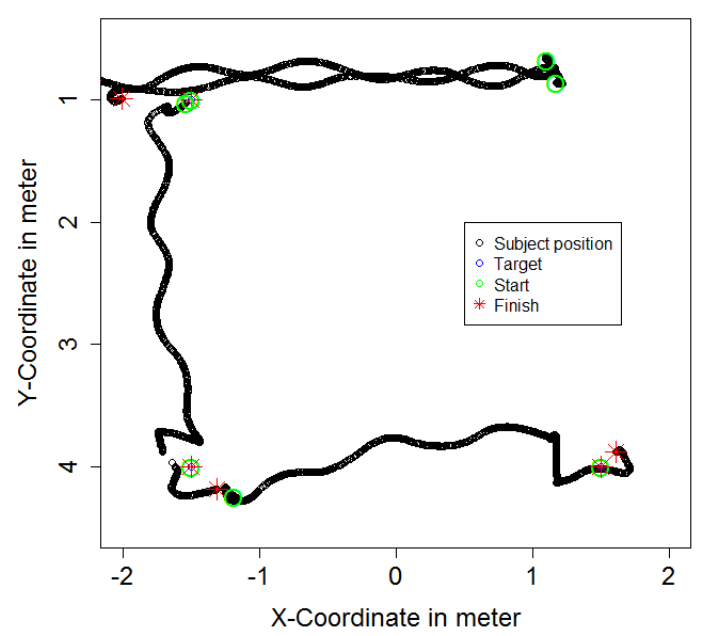

Figure 8.16.: All four curvature gains of one test subject with the value 0.00. It is plain to see that the general sense of direction towards the acoustic object was quite precise.

Proportional gain values and detections by test number

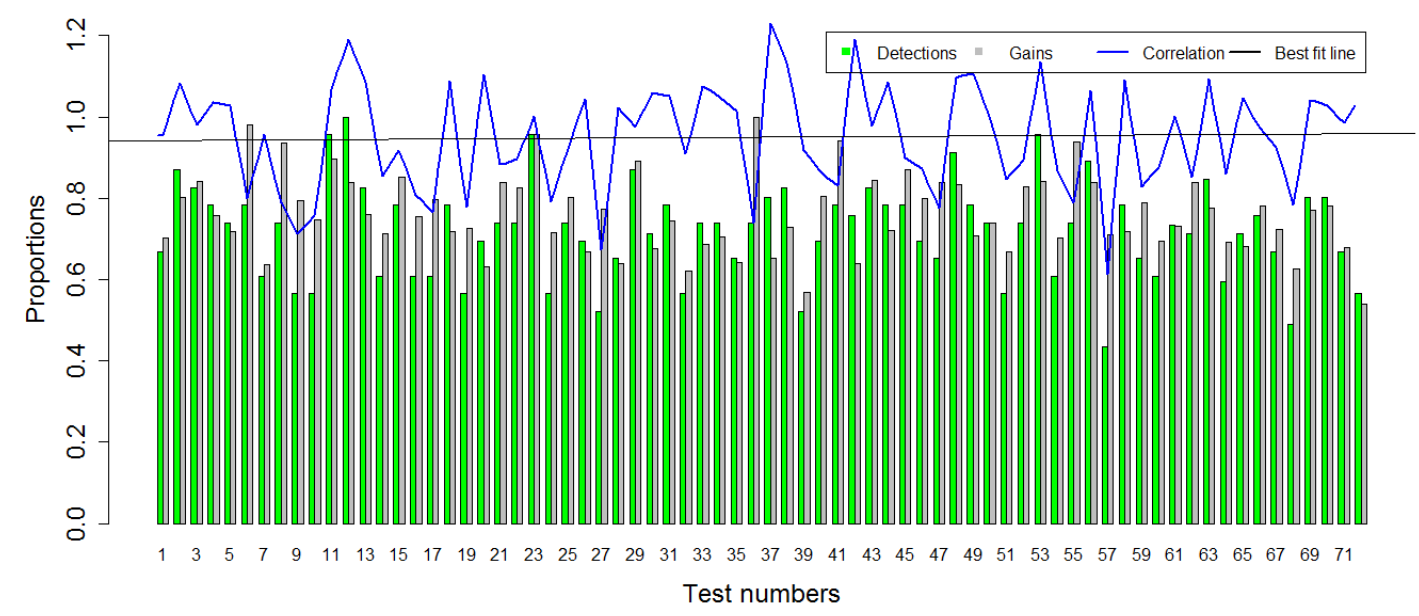

Figure 8.17.: Accumulated and normalized gain values by test number (grey) and normalized detection rates by test number (green). Both sets of data have been rescaled for better comparability. The best fit line indicates no trend during the course of the trials. 
Correlation between walking velocity and detection rate

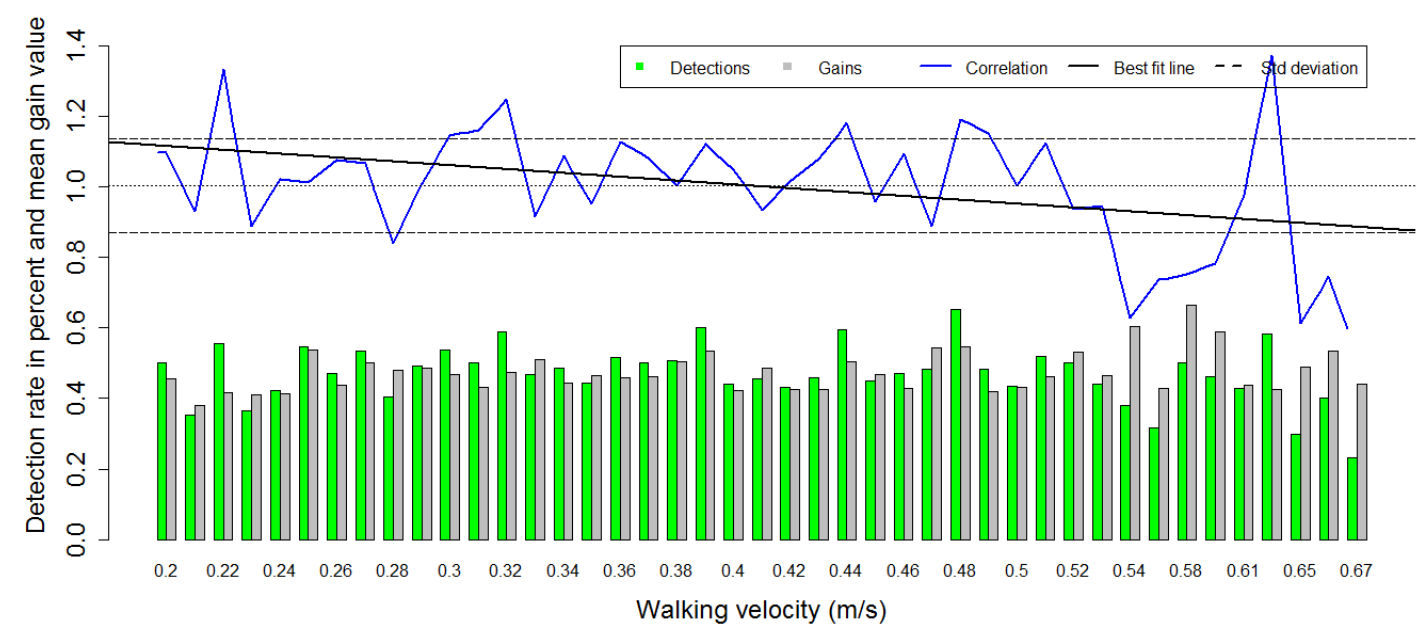

Figure 8.18.: Correlation between detection rate and walking velocity.

\subsection{Detection Rates by Test Number}

The question came up, whether or not the detection rate for manipulations would in- or decrease over the course of one set of trials, respectively over time within the $V E$, and exposure to the gains. Subjects may adapt to the manipulations over time, with a decreased detection rate as a result. On the other hand, test subjects may adapt to the $V E$ over time and as they gain more and more confidence, start to trust their instincts and senses more which might result in an increased detection rate.

Figure 8.17 illustrates the detection rates of all test groups aggregated by the position of the trial within the test sequence in green and the accumulated gain values for the corresponding test numbers in grey. Both sets of data have been rescaled for better comparison. For this rescaling the highest value within each data set defines the maximum value of 1.0. All other values within that data set are scaled accordingly. The correlation between gain value and detection rate had been obtained by dividing the detection rate with the gain value. The standard deviation of the correlation is 0.11 . The best fit line in black illustrates that the correlation did not change over time. No habituation effect could be detected. 


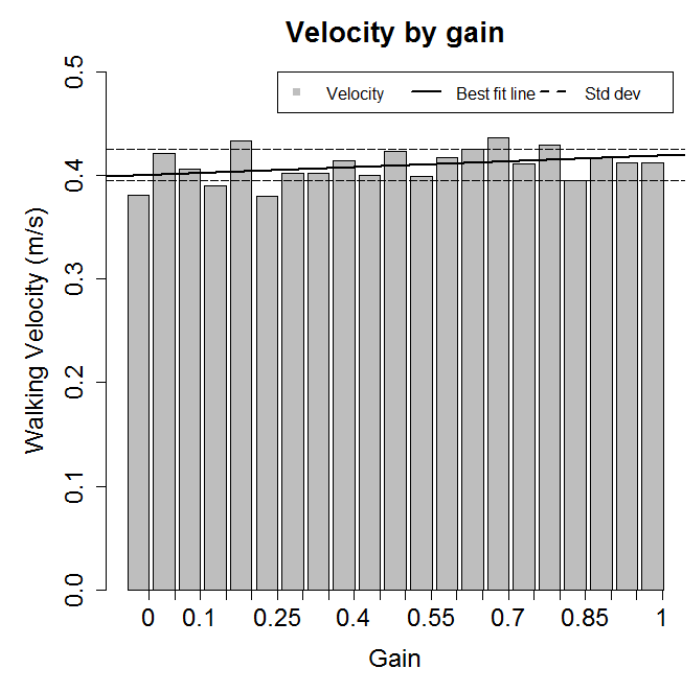

Figure 8.19.: The mean walking velocity Figure 8.20.: The mean walking velocity grouped by gain. The slight increase in walking velocity with increasing gains is within the standard deviation, so no trend could be observed.

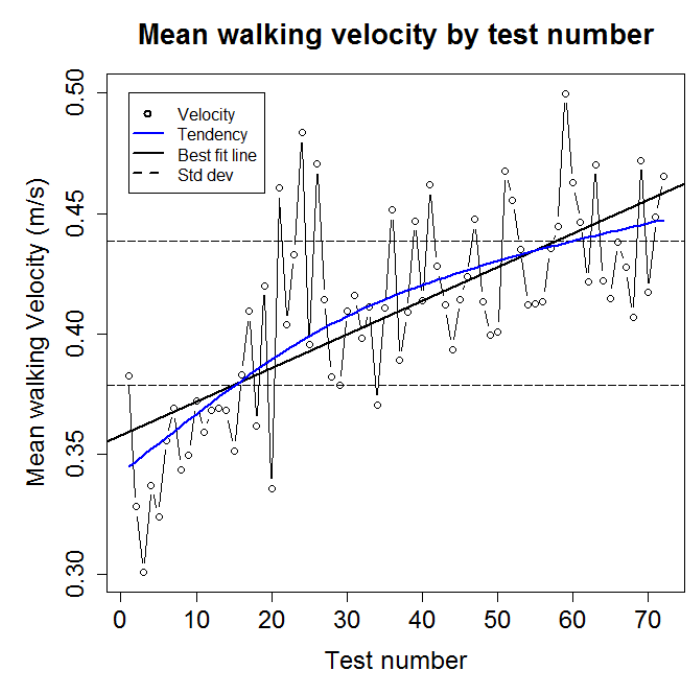
increased during the course of the trials.

\subsection{Velocity Dependency}

Multiple publications suggest that the velocity of users of a redirected walking application would affect the detection threshold of applied gains (Neth et al., 2012; Steinicke et al., 2008c).

Even though the position of each test subject was logged 60 times each second over the course of all trials, and the walking velocity between singular frames can be calculated with great precision, it is difficult to obtain meaningful average walking velocities. While the average walking velocity during all curvature gains was 0.41 meter per second, the average standard deviation of walking velocities was 0.3 meter per second. Standard deviation in this case describing for each path the deviation between single frames of the same path. Average standard deviation thus being the average of the standard deviations of all paths. The standard deviation of standard deviations, however, is with 0.18 meter per second comparably low. The walking velocities within each trial were very variable. Especially due to phases of reorientation, when subjects stopped to listen and concentrate on finding the precise direction towards the go-to target. To get closer to representative mean velocities, the first parts of each path, which was within 0.5 meters of the starting position, had not been included in the calculation of the 
velocity. At this point test subjects were considered to be on their way at travel speed. Still, with a standard deviation so high, the mean walking velocity is considered an approximation. Therefore any velocity related evaluations have to be considered with caution.

However, figure 8.18 shows the mean detection rates grouped by ranges of measured mean walking velocities and the correlation between detection rate and applied gain. The best fit line shows a decreasing tendency of the correlation with rising velocity. The standard deviation of the best fit line is 0.15 . Velocity ranges with less than 10 entries have been excluded from the evaluation.

Figure 8.19 shows the relationship between applied gain and walking velocities of curvature gains. No significant changes of the test subjects walking velocities could be measured in relation to the applied gains.

Figure 8.20 illustrates that the mean walking velocity increased steadily during the course of the trials. This suggests that the test subjects gained confidence during their time within the $V E$.

Neth et al. used visual feedback during their experiments regarding the relationship between walking velocity and detection rate, to help their test subjects to maintain a fixed velocity Neth et al. (2012). Through this technique they eluded the problem of large variation in velocity during individual tests. To apply this method to an acoustic $R D W$ experiment would provide a challenge however.

\subsection{Cyber Sickness}

As described in section 2.10, the stay within a IVE can have negative effects on the condition of the user. To measure possible effects of that regard, the $S S Q$ was conducted with each test subject right before and after the experiment. The measured and highest possible average total and sub scores are illustrated in table 8.4. The calculation of the scores is explained in section 2.10. The total average pre-SSQ score was 2.33 and the total average post- $S S Q$ score 14.0. The difference between pre- and post score is not severe in relation to the scale from 0 to 235.62. For example, in (Steinicke et al., 2009) even the pre score was with 16.3 higher than the post score of this experiment.

Some changes in symptoms that influence the oculomotor score like eye strain, difficulty focusing and blurred vision were observed, however, can most likely be traced back to the blindfold. No severe effects could be observed however, and the effects that were observed disappeared within minutes after the experiment. Not one test subject reported problems on 


\begin{tabular}{|c|c|c|c|c|c|}
\hline \multicolumn{2}{|l|}{ Pre score } & \multicolumn{2}{|l|}{ Post score } & \multicolumn{2}{|c|}{ Highest possible score } \\
\hline Total & $=2.33$ & Total & $=14$ & Total & $=235.62$ \\
\hline Nausea & $=0.82$ & Nausea & $=8.56$ & Nausea & $=200.34$ \\
\hline Oculomotor & $=1.1$ & Oculomotor & $=13.41$ & Oculomotor & $=159.18$ \\
\hline Disorientation & $=0.41$ & Disorientation & $=14.88$ & Disorientation & $=292.32$ \\
\hline
\end{tabular}

Table 8.4.: Simulator Sickness Questionnaire (SSQ) scores determined during the experiment.

their own. No experiment had to be aborted or interrupted, because of the condition of the test subject.

Overall the immersive virtual environment with wave field synthesis and redirected walking gains performs good regarding cyber sickness. 


\section{Conclusions}

Compared to the results of the other groups, the detection rate of group 1 (Naive) was very low, even though the test subjects performed distinctive turns during the trials. This led to the assumption that the subjects evaluated the probability of a mistake on their part higher than a faulting system. During the verbal feedback session after the completion of all trials, test subjects frequently gave indications towards them having had problems locating the objects during their trials. Considering the lack of these feedbacks in other groups, this further strengthens the assumption that the subjects were often aware of not walking on a straight path during curvature gains, but rather felt that they are disoriented in finding the object.

A comparison of the groups 1 (Naive) and 2 (Aware) show significant differences in correctly detected manipulations, while not so in the rate of false positives, even though the difference between these groups was only the introduction to the experiment and the resulting expectations. The difference suggests that test subjects of group 2 (Aware) were more likely to accuse the system of having moved the scene instead of blaming themselves. The lack of increase of false positives counters the consideration that the higher detection rate might be a result of increased likelihood of guessing and therefore must be considered as a more representative study for the perception of gains.

For group 3 (Expert), even though the detection rate again in significantly higher, a higher chance of the test subjects guessing the right answer has to be taken into account. The similarly increased rate of false positives supports this conclusion.

The groups 1 (Naive) and 3 (Expert) are considered special cases and group 2 (Aware) will be subject of further work and comparison.

The basic idea of $R D W$ is to make a user perceive external-motion within an immersive virtual environment as self-motion. For curvature gains this means to bend a straight virtual path into a curved physical path, while the user still perceives the physical path as a straight path as well. The experiment so far suggests that users, in a lot of cases, did not perceive their path as straight, but neither a manipulation. The uncertainty of their own capabilities of orienting themselves and finding their object within a sole acoustic IVE generates this option, which would probably not occur as severe in visual redirected walking application. Following 
this realization, I divide the range of gain values into three parts - green, red and grey. During green gains, test subjects perceive their path as straight. During red gains, test subjects detect the manipulation. During grey gains, test subjects are aware of walking a curved or crooked path, but still do not detect the manipulation. In (Steinicke et al., 2008a) test subjects had to walk on a $1 \mathrm{~m}$ wide pavement, but Steinicke et al. wrote that their test subjects "[...] left the pavement and had to reorient themselves [...]", and that they consequently "[...] tend to walk in a triangle rather than walking on an arc.". The same behaviour could be observed during a lot of trials with high value gains during the experiment presented in this work with great severity.

The detection threshold determined for group 1 (Naive) and group 2 (Aware) would be the threshold between the grey and red gains. During grey gains, test subjects did not indicate a manipulation, but in many cases felt some extent of frustration due to their difficulty in completing the trials. During green gains, test subjects seemed content with the situation. I assume that the same experiment with visual support would yield the detection threshold between grey and green gains. I propose to consider the difference between green and grey gains in further investigations.

This insight led to the generation of a fourth test group. Group 4 (two-alternative forcedchoice ( $2 A F C)$ ) had to complete the same trials, but after each applied gain, the test subject had to give a feedback for which they (as before) had two choices - right or left. This enforcement did no longer allow the test subjects to remain quiet after a trial, but forced them to either give the right or a false answer. When the test subjects did not perceive a manipulation, whether there was none (gain(0)) or the manipulation was too small to detect, they had to rely on their feeling or guess. As long as the test subjects did not perceive a manipulation, the answers should statistically be equally divided between "left" and "right". As the probability of the test subjects perceiving the manipulation increases, so should the discrepancy between "left" and "right" responses. This method of data acquisition should get results closer to the threshold between grey and green gains, because test subjects who had to constantly correct their path to the "left" should very unlikely follow up that trial with a "right" response.

At low gains and the $25 \%$ detection threshold for curvature gains no significant difference could be registered between group 2 (Aware) and group 4 (2AFC). The detection threshold actually seems a little lower, but this could be traced back to the quite small test groups and generally a very low chance of detection. The $50 \%$ detection threshold for curvature gains however was reached at lower gains in group 4 (2AFC) compared to group 2 (Aware) $\left(g_{C}(0.275)\right.$ $\equiv 3.63 \mathrm{~m}$ to $\left.g_{C}(0.4) \equiv 2.5 \mathrm{~m}\right)$ and the maximum detection threshold for curvature gains was a lot higher in group $4(2 A F C)(\sim 90 \%$ to $\sim 65 \%)$. For rotation gains all thresholds and the maximum 
detection rate of group $4(2 A F C)$ were higher as well. This suggests that the $2 A F C$ task version got closer to the threshold between grey and red gains. However, some trials remained, in which the test subject noticed the bent physical path, but still did not give the right feedback. Probably due to characteristics of the acoustic orientation that would not apply to a visual $V E$. One resulting hypothesis is that humans in general are more content with perceiving sound sources from incorrect directions, due to the characteristics of acoustic reflections. Depending on the environment, sound waves can be reflected around corners or even thrown back towards their origin in such a high quality, which prevents listeners from detecting the reflection. Visually this only happens in very special environments like in labyrinths of mirrors. Under those circumstances visitors can become confused about the origin of an object, which they are trying to locate visually, due to the visual reflections.

The fact that the detection threshold of negative rotation gains (the VE rotates in the opposite direction) is significantly lower than the threshold for positive rotation gains (the VE rotates with the user) could be explained by different circumstances other than really the test subjects just being more prone to this kind of manipulation. The virtual rotations within this experiment were always $180^{\circ}$. Only the physical rotation was influenced by the gain. Since the manipulation of the $V E$ is linearly dependent on the physical movements, a greater physical rotation does result in a more extensive accumulated manipulation. For a positive rotation gain of e.g. 0.5 (50\%), the physical rotation of $180^{\circ}$ causes a $V E$ rotation of $90^{\circ}$. The test subjects had to cover these additional $90^{\circ}$ physical rotation, which resulted in another $45^{\circ}$ of $V E$ rotation, which in the end amounts to a $V E$ rotation of $180^{\circ}$ and a total physical rotation of $360^{\circ}$. For a rotation gain of $-0.5(-50 \%)$, the physical rotation would be $120^{\circ}$, and the manipulation therefore only $60^{\circ}$. The more extensive total manipulation to the $V E$ and larger amount of time exposed to the gain might influence the detection threshold.

The results of all groups also suggest that the detection rate is considerable dependent on the context and various circumstances. The comparison between group 1 (Naive) and the other groups shows that the knowledge of the system has a significant impact on the detection rate. Close proximity to the go-to target seemed to have had an impact on the detection probability as well. This fits previous findings of Steinicke et al. who write that

"Informal tests have motivated that manipulations can be intensified in some cases, e. g., when less objects are close to the camera, which could provide further motions cues while the user walks." (Steinicke et al., 2009)

Therefore an acoustic RDW application might benefit from lower curvature gains, when the user closes in on objects. Even though the evaluation of this experiment regarding walking velocities is not very strong, a steady increase in velocity over the course of the trials could be 
measured, which, according to related work (Neth et al., 2012), should come with an increase in detection probability. This might also apply to visual IVEs, but likely not as severe. Due to the impact of numerous characteristics of different experiments, the comparison of results of different experiments is also difficult. The comparison of this work and (Serafin et al., 2013) (illustrated in tables 8.2 and 8.3) show that some results are very close to each other. The identified difference between the capability to up-scale a rotation only differs by $\sim 2.3 \%$. While test subjects of this experiment, however, head great problems in identifying downscaled rotations at all, during Serafins experiment, the detection rate was even higher. For curvature gains, the detection rate was higher as well, but this experiment showed that the results are highly dependent on a variety of factors, which were probably different between the experiments. 


\section{Summary and Future Work}

This chapter will give a brief summary over the contents of this, and a glimpse into future work.

\subsection{Summary}

The goal of this thesis was the identification of detection thresholds for acoustic RDW applications and characteristics that are particular to acoustic $R D W$ in comparison to visual $R D W$. To this end an introduction into $R D W$ in general, the appliance of gains to manipulate users' movements, and some particular aspects like physical objects in IVEs, velocity dependency, $R O T s$, non-visual $R D W$ and cyber sickness have been given. After an introduction into WFS and tracking systems, the laboratory, the $M o W e C$ and its $R D W$ test extension have been illustrated.

The choice of gains to be part of the experiment and the experiment design have been illustrated in detail. An acoustic IVE had been designed to be played back by a WFS system to give the test subjects a context to navigate through. While the test subjects of four different test groups performed a randomized and automated sequence of trials, their movements and verbal responses to the manipulations had been logged.

After the completion of all trials, the results were evaluated towards different problems. The evaluation showed a strong dependency between the detection thresholds and the test subjects' knowledge of the experiment. Detection thresholds for rotation gains and curvature gains have been identified for the different groups. It was found that many participants with little knowledge, but also some with better knowledge could be fooled into very high manipulations which is probably due to the fact, that they are not as accustomed to navigating by acoustic cues and that even in reality, due to the reflections, sound sources are sometimes perceived at wrong locations. The detection rate, however, did not change during the course of the experiment, while the walking velocity did increase, and almost no effects of cyber sickness could be observed. The results also suggest that the detection thresholds are dependent on a variety of factors of the experiment design and therefore a direct comparison of the results to previous visual $R D W$ experiments would not yield very meaningful results. However, acoustic $R D W$ seems promising and worthy of further investigation. 


\subsection{Future Work}

Even though the $V E$ for this experiment was equipped with multiple virtual sound source, the focus of the test subjects was primarily on the current objective which was placed for each trial within the $V E$ without a direct context. The test subjects therefore did not have to have a cognitive model of the $V E$. Such an model would increase the perceived stability of the $V E$ however, and could give the test subjects more confidence in their ability to navigate in an acoustic environment. A future experiment within a constant IVE, in which permanent objects are chosen as objects, could therefore yield interesting results. The choice of curvature gain and object for each trial would have to be chosen carefully though, to keep the translation between the physical WFS area and IVE from becoming too large. For rotation gains no special care would have to be pain on that regard.

Another method, which could increase the confidence of test subjects, could be a training phase before the trials with direct accuracy feedback. Test subjects would get a series of trials without the appliance of any gains and would get a feedback about their precision directly after each trial. Through this, the test subjects should get a better sense about their capabilities. In case a $R D W$ experiment follows this training, the comparison of the results of subjects who had the training and those who did not have the training could be interesting.

As mentioned in the conclusions, the distinct difference between detection thresholds of upscaling and down-scaling rotation gains could be produced by the difference in the accumulated manipulations. An experiment in which the physical - instead of the virtual - rotation would stay the same between trials could illuminate this hypothesis.

Different previous work mention, that $R D W$ works best with rotation gains and that curvature gains would be used mostly just in castes, in which the user does not rotate his or her head enough. Even though, experiments only investigate rotation gains while stationary rotating. Experiments should be conducted to investigate the appliance of rotation gain while the test subjects are walking and are stimulated to turn heir heads. Crossing a street on a cross-walk could be a fitting scenario.

For a best possible comparison between acoustic and audiovisual $R D W$, Meyer is already working on a bachelor thesis to repeat the experiment conducted for this work with an additional visual component via an Oculus Rift ${ }^{1}$ (Meyer, 2015).

\footnotetext{
${ }^{1}$ https://www.oculus.com/en-us/
} 


\section{A. Functions}

A.1. Rotation Gains $\left(g_{R}\right)$

$$
\begin{gathered}
g_{R}=\frac{R_{\text {physical }}-R_{\text {virtual }}}{R_{\text {physical }}} \\
R_{\text {virtual }}=R_{\text {physical }}-R_{\text {physical }} \cdot g_{R} \\
R_{\text {physical }}=\frac{R_{\text {virtual }}}{1-g_{R}}
\end{gathered}
$$

With:

$g_{R} \quad:=$ Rotation gain

$R_{\text {physical }}:=$ The physical rotation in degree

$R_{\text {virtual }} \quad:=$ The virtual rotation within the IVE in degree 
A.2. Curvature Gains $\left(g_{C}\right)$

$$
\begin{gathered}
g_{C}=\frac{1}{r} \\
r=\frac{1}{g_{C}} \\
R_{R}=m \cdot g_{C} \\
g_{C}=\frac{R_{D} \cdot 0.0174532925}{m} \\
r=\frac{1}{\frac{R_{D} \cdot 0.0174532925}{m}}
\end{gathered}
$$

With:

$g_{C} \quad:=$ Curvature gain

$r \quad:=$ The radius of the circle defined by the curve in meters

$m \quad:=$ The length of the path in meters

$R_{R} \quad:=$ The rotation of the user in radiant after a path of length $m$

$R_{D} \quad:=$ The rotation of the user in degree after a path of length $m$ 


\section{B. Test subjects}

\begin{tabular}{llll|llll} 
Nr & Group & Gender & Age & Nr & Group & Gender & Age \\
\hline 1 & 1 & w & 24 & 21 & 1 & w & 45 \\
2 & 2 & w & 21 & 22 & 1 & $\mathrm{~m}$ & 38 \\
3 & 2 & $\mathrm{w}$ & 27 & 23 & 2 & $\mathrm{~m}$ & 27 \\
4 & 2 & $\mathrm{~m}$ & 21 & 24 & 2 & $\mathrm{~m}$ & 22 \\
5 & 2 & $\mathrm{~m}$ & 26 & 25 & 2 & $\mathrm{~m}$ & 32 \\
6 & 3 & $\mathrm{~m}$ & 26 & 26 & 1 & $\mathrm{~m}$ & 24 \\
7 & 2 & $\mathrm{~m}$ & 27 & 27 & 3 & $\mathrm{~m}$ & 24 \\
8 & 2 & $\mathrm{w}$ & 25 & 28 & 1 & $\mathrm{~m}$ & 23 \\
9 & 2 & $\mathrm{w}$ & 23 & 29 & 1 & $\mathrm{~m}$ & 23 \\
10 & 2 & $\mathrm{~m}$ & 53 & 30 & 4 & $\mathrm{~m}$ & 22 \\
11 & 3 & $\mathrm{~m}$ & 31 & 31 & 2 & $\mathrm{~m}$ & 24 \\
12 & 2 & $\mathrm{~m}$ & 26 & 32 & 2 & $\mathrm{w}$ & 22 \\
13 & 1 & $\mathrm{~m}$ & 27 & 33 & 3 & $\mathrm{~m}$ & 60 \\
14 & 1 & $\mathrm{~m}$ & 49 & 34 & 4 & $\mathrm{w}$ & 24 \\
15 & 1 & $\mathrm{~m}$ & 66 & 35 & 4 & $\mathrm{~m}$ & 23 \\
16 & 4 & $\mathrm{~m}$ & 21 & 36 & 4 & $\mathrm{~m}$ & 26 \\
17 & 2 & $\mathrm{~m}$ & 21 & 37 & 4 & $\mathrm{w}$ & 22 \\
18 & 4 & $\mathrm{~m}$ & 21 & 38 & 4 & $\mathrm{~m}$ & 22 \\
19 & 2 & $\mathrm{~m}$ & 31 & 39 & 4 & $\mathrm{~m}$ & 25 \\
20 & 2 & $\mathrm{w}$ & 54 & & & &
\end{tabular}

Table B.1.: Test subjects 


\section{List of Figures}

2.2. A user wearing the $H M D$ and the wide area tracker during the experiments for (Razzaque et al., 2001). . . . . . . . . . . . . . . . . . . . .

2.1. Two pictures of the fire drill experiment in (Razzaque et al., 2001). Crosses denote static waypoints. . . . . . . . . . . . . . . . 6

2.3. The algorithm for computing the rotational distortion rate. Figure taken form (Razzaque et al., 2001). . . . . . . . . . . . . . . . . . . .

2.4. Illustrations of the four RDW algorithms tested in (Hodgson and Bachmann, 2013). Users were steered towards particular targets or onto a target path. Figure taken from (Hodgson and Bachmann, 2013). . . . . . . . . . . . . 10

2.5. The curvature gain bends a path and the rotation gain scales a rotation. . . . . 11

2.6. Illustration of 5 different curves defined by the given values. . . . . . . . . . . 12

2.7. Evaluation of the generic redirected walking concepts for (a) rotation gains $g_{R}[u]$ and (b) curvature gains $g_{C}[w]$. The bars indicate how much the users have perceived the manipulated walks. The horizontal lines indicate the detection thresholds. (Steinicke et al., 2008c, 2009) _ . . . . . . . . . . . . . . . 13

2.8. Illustration of a user's path during a experiment in (Steinicke et al., 2008c) showing (left) path through the physical setup and (right) virtual path through the $V E$ and positions at different points in time $t_{0}, \ldots, t_{4}$. (Steinicke et al., 2008c) 14

2.9. Two figures showing how a physical table serves as a block of stone in a $V E$ (Steinicke et al., 2008c) . . . . . . . . . . . . . . . . . 15

2.10. Curvature detection thresholds across all participants (corresponding radius in brackets, bars denote standard error of the mean). (Neth et al., 2012) . . . . . . 16

2.11. Two different approaches to ROTs to prevent a user from walking outside the tracking area. (Neth et al., 2012) . . . . . . . . . . . . . . . . . . . 17 
2.12. Two figures showing the results of the $2 A F C$ experiments in (Serafin et al., 2013). In the $2 A F C$ experiment, subjects had to give one of two possible answers after each test. For (a) it was "greater" or "smaller". For (b) is was "left" or "right". These figures show the proportion of one of these answers to the other one. . . . . . . . . . . . . . . . . . . . . . . . . 19

3.1. The creation of a synthetic sound wave (synthetic wave audio products $\mathrm{GmbH}$, 2015) . . . . . . . . . . . . . . . . . . . . . 24

3.2. Layout of the WFS system based on (Four Audio GmbH, 2011). . . . . . . . 26

3.3. Exemplary WFS layout displayed by $x W O N D E R \ldots \ldots \ldots$

5.1. Layout of the laboratory with 26 audio modules and 6 infrared cameras. . . . 33

5.2. Processing sequence of the $\mathrm{MoWeC} \ldots \ldots \ldots \ldots$. . . . . . . . . 34

5.3. The GUI for the $R D W$ component. . . . . . . . . . . . . . . . . . . . 35

7.1. The height of virtual sound sources is dependent on the relative position of the auditor to the relevant speakers. While the auditor moves closer to the speaker, the red virtual sound source will stay between the head and the speaker and the blue virtual sound source behind the speaker, and such changing their heights

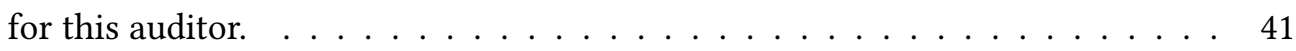

7.2. All possible starting positions for curvature gain and rotation gain tests, with corresponding orientations. . . . . . . . . . . . . . . . . . . . 44

7.3. During the automated tests, several states will be passed in a loop. At the start of each loop, a test is chosen randomly. Next the test person will be directed to the starting position of the test and then be aligned into the starting direction. Depending on the current test, the test setup will be set and then the actual test will be started and executed until the test subject fulfilled the conditions. To wrap each test up, further logging and deactivation of sounds is necessary before determining, whether any more tests are left. . . . . . . . . . . .

8.1. An example of a curvature gain trial with $g_{C}(0.4)$. The length of both paths is 3 meters. The ideal physical path is a segment of a circle with a radius of 2.5 meters, which changes the direction by $70^{\circ}$ after 3 meters. . . . . . . . . . 46

8.2. Gain detections over all test subjects. The applied values on the $\mathrm{x}$ axis and the observed detection rate on the y axis. . . . . . . . . . . . . . . . 48 
8.3. Curvature gain detections with respect to the gain being positive or negative (bending the path to the left or right) divided by groups. No consistent trend can be observed while comparing the detections of positive and negative gains. 50

8.4. Curvature gain detections over all test subjects with respect to the gain being positive or negative. . . . . . . . . . . . . . . . . 50

8.5. Gain detections of group 1 (Naive). The applied values on the $\mathrm{x}$ axis and the observed detections on the y axis. . . . . . . . . . . . . 51

8.6. Gain detections of group 2 (Aware). The applied values on the $\mathrm{x}$ axis and the observed detections on the y axis. . . . . . . . . . . . . . . 52

8.7. Gain detections of group 3 (Expert). The applied values on the $\mathrm{x}$ axis and the observed detections on the y axis. . . . . . . . . . . . . . . . 54

8.8. Gain detections of test subject 26/27. The same subject participated first in group 1 (Naive) as subject 26 and later in group 3 (Expert) as subject 27. The difference between figures $8.8 \mathrm{a} \& 8.8 \mathrm{~b}$ and $8.8 \mathrm{c} \& 8.8 \mathrm{~d}$ are results of only the increased knowledge of the experiment when participating in group 3 (Expert).

8.9. Gain detections of test subject 5/6. The same subject participated first in group 2 (Aware) as subject 5 and later in group 3 (Expert) as subject 6. The difference between figures $8.9 \mathrm{a} \& 8.9 \mathrm{~b}$ and $8.9 \mathrm{c} \& 8.9 \mathrm{~d}$ are results of only the increased knowledge of the experiment when participating in group 3 (Expert). . . . . . 56

8.10. Gain detections of group $4(2 A F C)$. The applied values on the $\mathrm{x}$ axis and the

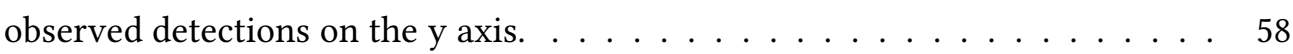

8.11. Two paths during trials with very high curvature gains which nonetheless resulted in a wrong (or no) detection.

8.12. Two paths, during which the test subject walked a comparatively even curve and did not perceive the manipulation. The test subject gave the wrong answer within the $2 A F C$ trial, so the manipulation was not detected. . . . . . . . .

8.13. Two paths, in which the test subjects did not perceive a manipulation, even though the manipulation was severe (curvature gain $(1.0)=>$ a radius of 1 meter) and the subjects were aware of their crooked path. . . . . . . . .

8.14. The two paths of figure 8.13 speculatively modified, as they might have been

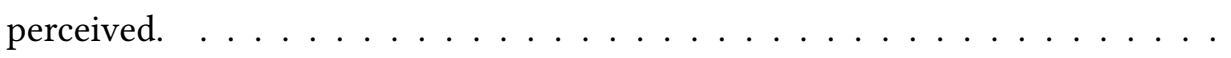

8.15. Two paths, in which the test subject tried to retrace his steps to the starting position to restart the trial. Note the rescaled coordinate system compared to other path plots. . . . . . . . . . . . . . . . . . . . 
8.16. All four curvature gains of one test subject with the value 0.00 . It is plain to see that the general sense of direction towards the acoustic object was quite precise. 65

8.17. Accumulated and normalized gain values by test number (grey) and normalized detection rates by test number (green). Both sets of data have been rescaled for better comparability. The best fit line indicates no trend during the course

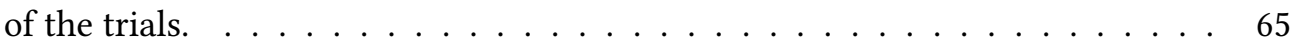

8.18. Correlation between detection rate and walking velocity. . . . . . . . . . 66

8.19. The mean walking velocity grouped by gain. The slight increase in walking velocity with increasing gains is within the standard deviation, so no trend could be observed. . . . . . . . . . . . . . . . . . . 67

8.20. The mean walking velocity increased during the course of the trials. 


\section{Glossary}

MoWeC source The $\mathrm{MoWeC}$ source is the primary data type within the $\mathrm{MoWeC}$, which represents a virtual sound source. $33,35,83$

MoWeC The $\mathrm{MoWeC}$ was initially developed during my bachelor thesis and hence been extended and overhauled. It's main purpose is the connection between a tracking system and the WFS system, while defining a fundamental process, providing a number of classes and methods and a section for optional components, where application logics can be implemented. $32-35,74,80,83,85,86,88$

acoustic $\boldsymbol{R} \boldsymbol{D} \boldsymbol{W} R D W$ by means of solely acoustic cues. $3,68,74$

acoustic $\boldsymbol{R} \boldsymbol{D} \boldsymbol{W}$ application An redirected walking application by means of solely acoustic cues. $2,3,36,72,74$

ART tracker The tracking system of the company "Advanced Realtime Tracking (ART) GmbH". 30

curvature gain A curvature gain maps a real world translation to a rotation within the IVE. While a user walks forward (i. e.), the world rotates around her and such giving her the impression, that she deviates from her path by self-motion, to stimulate her to walk a curve while staying under the impression maintaining her path straight forward. 11, 12, $20,36,37,40-44,46,48-65,67,68,70-75,77,79-82,84$

cWONDER CWONDER is the central communication module of sWONDER. 26-28, 83

cyber sickness Cyber sickness initially appeared to be similar to simulator sickness but differs in predominant symptoms and severity Stanney et al. (1997). 3, 4, 20, 21, 68, 69, 74, 83

displacement gain A displacement gain maps a real world rotation to a translation within the IVE. 12, 36 
elementary wave The sound wave a single channel of the WFS system emits. Multiple elementary waves together form the synthetic wave. $24,25,84,86$

external-motion external-motion describes the motion of one or multiple objects in relation to the (non-)motion of a subject and stands in opposition to self-motion, where the subject itself moves. Under certain circumstances external-motion can be perceived as self-motion and vice verse. 5, 6, 17, 21, 38, 70, 84, 86

focused sound source A focused sound source within the WFS system is a point sound source with a position within the physical WFS area. Focused sound sources pose a difficulty, because auditors could be located between the virtual sound source and the speaker modules, that emit the sound. 44,84

gain Gains apply modifications to users movements. 5, 8, 10-12, 18, 36, 43, 45, 46, 48-58, 60, $61,65-72,74,75,80-82,84$

go-to target The go-to target is the virtual sound source, that animates a user to walk into a certain direction or to a certain position by being placed on a position in that direction and playing an distinctive sound. It is the object during curvature gain trials. 39, 42, 43, $56-59,61-64,67,72,84,85$

group 1 (Naive) The first test group, whose subjects had no idea, that they were participating in a $R D W$ experiment. $44,48,50,51,53,55,56,60,62,70-72,81$

group 2 (Aware) The second test group, whose subjects knew, that they were participating in a $R D W$ experiment, but did not know how exactly the experiment was designed. 44 , $45,48,50,52,53,55-57,60,62,70,71,81$

group 3 (Expert) The third test group, whose subjects know, that they were participating in a $R D W$ experiment, and had a very good understanding of the test procedure. $44,48,50$, $53-56,60,70,81$

group $4(2 A F C)$ The fourth test group, whose subjects know, that they were participating in a $R D W$ experiment, just as the subjects of group 2, but had to give a feedback after each trial in the manner of a $2 A F C$ task. $45,48,50,55-58,60,61,71,72,81$

human locomotion triple The human locomotion triple $(s, u, w)$ consists of three normalized vectors. $u$ represents the head orientation, $w$ the walking direction and $s$ is orthogonal to $w$ and parallel to the walk plane. Through the HLT gains can be applied to a users movement. $9,84,85,88$ 
linear sound source A linear sound source within the WFS system represents the origin of a sound (see virtual sound source). The linear sound source, in comparison to a point sound source, defines a plane wave and such merely a direction from where the sound is coming from instead of a fixed position. The position of the linear sound source is therefore meaningless, but the orientation defines the characteristics. 25, 39, 85

locomotion triple The locomotion triple was introduced in (Steinicke et al., 2008b) and evolved into the human locomotion triple (HLT). 9

motion sickness a feeling of nausea and dizziness, sometimes accompanied by vomiting, resulting from stimulation by motion of the semicircular canals of the ear during travel by car, plane, etc. motion sickness (2015). 21

object The object within this $R D W$ experiment is the go-to target or turn-to target, depending on the current gain. $45,56,57,59,64,65,70,75,82,84,85$

open sound control A communication protocol for computers, synthesizers and other multimedia components via ethernet. 26,88

optional component The optional component is the part of the $M o W e C$, where optional application logics can be implemented. 33, 35, 85

physical WFS area The physical WFS area is the area, in which listeners can be located. It is generally a subset of the virtual WFS area. $23,25,40,42,44,53,75,84,85,87$

point sound source A point sound source within the WFS system represents the origin of a sound (see virtual sound source). The virtual sound is emitted in an even circular manner and will be rendered for the physical WFS area accordingly. The linear sound source poses the alternative. $25,39,84,85$

redirected walking application An application using $R D W$ techniques. 2, 3, 6, 31, 36, 44, $67,70,83,86$

rotation gain A rotation gain scales the real world rotation of the user and such increasing or decreasing the resulting rotation in the IVE to give the user the impression, that her real rotation was greater (respectively less) then it really was. 11, 12, 18, 36, 37, 40-44, $46,48,49,51,52,54-58,60,71,72,74-76,79,80$ 
self-motion "Movement caused by oneself or itself, not by an external action or agent." Stevenson (2010). In opposition to external-motion, where an object is moving in relation to oneself. self-motion and external-motion can result in the same relative motion between the subject and the object. Under certain circumstances external-motion can be perceived as self-motion and vice versa. 5, 6, 13, 16, 17, 36, 38, 70, 83, 84, 86

simulator sickness Simulator sickness describes the phenomenon, that users of immersive simulators may experience symptoms such as dizziness or nausea. The reasons are the same as with sea sickness. Different cues for orientation and motion are not consistent with one and another. $6,20,21,31,83,86$

simulator sickness questionnaire The $S S Q$ was developed by Kennedy et al. in 1993 to measure simulator sickness Kennedy et al. (1993). 20, 88

sWONDER Modular software packet for the control and rendering of WFS systems. 26, 83, 87

synthetic sound wave A synthetic sound wave is created by a WFS system, by precisely timing the emission of elementary waves. $24,80,86$

time-dependent gain A time-dependent gain maps elapsed time to virtual rotations or translations and such manipulates the $V E$ without respect to the user movements. 11, 12, 36, 86

time-dependent rotation gain A time-dependent rotation gain maps elapsed time to virtual rotations and such manipulates the $V E$ without respect to the user movements. 12, 36, 86

time-dependent translation gain A time-dependent translation gain maps elapsed time to virtual translations and such manipulates the $V E$ without respect to the user movements. 36,86

TrackerListener Application The TrackerListener Application is an external component for the $\mathrm{MoWeC}$, which forwards and if necessary format tracking data from an tracking system. Through the use of this external component, the $M o W e C$ gains a lose coupling to the tracking system. This is especially useful, when exchanging the tracking system. 33,86

tracking area The tracking area is the physical space, that is covered by the tracking system. In redirected walking applications the tracking area is the area, in which the user can physically move, while the $V E$ is the area in which she virtually moves. 7, 8, 11, 16, 17, $32,53,63,79,86$ 
tracking system A computer system that registers the position and depending on the system the orientation of objects within a defined space. 74

translation gain A translation gain scales the real world movement of the user and such increasing or decreasing the distance in the IVE in respect to the same real world translation. 11, 36, 37

turn-to target The turn-to target is the virtual sound source, that animates a user to orient themselves into a certain direction by being placed on a position in that direction and playing an distinctive sound. $39,42,43,57,85,87$

tWONDER $t W O N D E R$ is the rendering unit of $s W O N D E R .27,87$

virtual WFS area The virtual WFS area is the area, in which virtual sound source can exist. It is generally a super set of the physical WFS area. 85,87

virtual sound source A virtual sound source within the WFS system is a virtual object, that represents the origin of sounds, that are mapped to it. Sounds, that are played back via this virtual sound source are rendered by the WFS system in such a way, that they appear to be coming from the position of the virtual sound source. 23, 25-28, 36, 39-41, 43, 44, $75,80,83-85,87$

xWONDER $x$ WONDER is the GUI component of sWONDER. 26-28, 80, 87 


\section{Acronyms}

MoWeC Motion Tracker-Wave Field Synthesis-Connector. 32-35, 74, 80, 83, 85, 86

WFS system wave field synthesis system. 23-27, 32, 33, 35, 37, 39, 40, 74, 80, 83-87

2AFC two-alternative forced-choice. $19,45,48,50,55-61,71,72,80,81,84$

DAW digital audio workstation. 26, 32, 33, 35

GUI graphical user interface. 26, 27, 35, 80, 87

HCI human computer interface. 1

HLT human locomotion triple. 9, 11, 84, 85

HMD head mounted display. 5, 18, 79

IVE immersive virtual environment. 1-3, 6, 11, 14, 17, 38, 44, 68-70, 73-76, 83, 85, 87

MR mixed reality. 1

MSQ motion sickness questionnaire. 20

OSC open sound control. 26-28, 33, 35

PSE point of subjective equality. 18, 20

RDW redirected walking. 1-5, 7-9, 13, 15-18, 20, 21, 31, 35, 36, 38, 40, 44, 68-70, 72, 74, 75, $80,83-85$

ROT reorientation technique. $8,16,17,74,79$

SSQ simulator sickness questionnaire. 20-22, 68, 69, 86 
UAS Hamburg University of Applied Sciences Hamburg. 3

VBAP vector base amplitude panning. 18

VE virtual environment. 3, 4, 6-9, 11, 14-18, 21, 36, 39, 44, 45, 56, 59, 62, 63, 66, 68, 72, 75, 79, 86

VR virtual reality. 1

WFS wave field synthesis. $2,3,23-28,32,33,35,37-40,42,44,53,69,74,75,80,83-88$

WONDER Wave field synthesis of new dimensions of electronic music in realtime. 26, 27 


\section{Bibliography}

Andresen, G. (2002). Playing by ear: Creating blind-accessible games. Gamasutra Article.

Baalman, M., Hohn, T., Schampijer, S., and Koch, T. (2007). Renewed architecture of the sWONDER software for a wave field synthesis on large scale systems. Linux Audio Conference 2007.

Baalman, M. and Plewe, D. (2005). WONDER - a software interface for the application of Wave Field Synthesis in electronic music and interactive sound installations. 3rd International Linux Audio Conference.

Baalman, M. A. J. (2008). On wave field synthesis and electro-acoustic music, with a particular focus on the reproduction of arbitrarily shaped sound sources.

Beimler, R., Bruder, G., and Steinicke, F. (2013). Immersive Guided Tours for Virtual Tourism through 3D City Models.

Berthoz, A. (2002). The brain's sense of movement. Harvard University Press.

Bouguila, L. and Sato, M. (2002). Virtual locomotion system for large-scale virtual environment. In Virtual Reality, 2002. Proceedings. IEEE, pages 291-292. IEEE.

Bridgeman, B., Van der Heijden, A., and Velichkovsky, B. M. (1994). A theory of visual stability across saccadic eye movements. Behavioral and Brain Sciences, 17(2):247-257.

Brooks Jr, F. P., Airey, J., Alspaugh, J., Bell, A., Brown, R., Hill, C., Nimscheck, U., Rheingans, P., Rohlf, J., Smith, D., et al. (1992). Six generations of building walkthrough: Final technical report to the National Science Foundation.

Bruder, G., Steinicke, F., Hinrichs, K., and Lappe, M. (2009). Reorientation During Body Turns. In Proceedings of the 15th Foint Virtual Reality Eurographics Conference on Virtual Environments, JVRC’09, pages 145-152, Aire-la-Ville, Switzerland, Switzerland. Eurographics Association. 
Chance, S. S., Gaunet, F., Beall, A. C., and Loomis, J. M. (1998). Locomotion Mode Affects the Updating of Objects Encountered During Travel: The Contribution of Vestibular and Proprioceptive Inputs to Path Integration. Presence: Teleoper. Virtual Environ., 7(2):168-178.

Cratty, B. J. (1966). Perceptual thresholds of non-visual locomotion. Part II. Los Angeles, Dept. of Physical Education, Univ. of California.

Darken, R. P., Cockayne, W. R., and Carmein, D. (1997). The omni-directional treadmill: a locomotion device for virtual worlds. In Proceedings of the 10th annual ACM symposium on User interface software and technology, pages 213-221. ACM.

Dichgans, J. and Brandt, T. (1978). Visual-Vestibular Interaction: Effects on Self-Motion Perception and Postural Control. In Held, R., Leibowitz, H., and Teuber, H.-L., editors, Perception, volume 8 of Handbook of Sensory Physiology, pages 755-804. Springer Berlin Heidelberg.

Engel, D., Curio, C., Tcheang, L., Mohler, B., and Bülthoff, H. H. (2008). A Psychophysically Calibrated Controller for Navigating Through Large Environments in a Limited Free-walking Space. In Proceedings of the 2008 ACM Symposium on Virtual Reality Software and Technology, VRST '08, pages 157-164, New York, NY, USA. ACM.

Field, T. and Vamplew, P. (2004). Generalised algorithms for redirected walking in virtual environments. PhD thesis, University of Tasmania.

Floros, A. and Tatlas, N.-A. (2011). Spatial enhancement for immersive stereo audio applications. In Digital Signal Processing (DSP), 2011 17th International Conference on, pages 1-7. IEEE.

Fohl, W. (2013). The wave field synthesis lab at the HAW Hamburg. In Sound-PerceptionPerformance, pages 243-255. Springer.

Fohl, W. and Nogalski, M. (2013). A Gesture Control Interface for a Wave Field Synthesis System. In Proceedings of the International Conference on New Interfaces for Musical Expression (NIME).

Four Audio GmbH (2011). Kurze Einführung: Wellenfeldsynthese-Software und -Cluster.

Frissen, I., Campos, J. L., Sreenivasa, M., and Ernst, M. O. (2013). Enabling unconstrained omnidirectional walking through virtual environments: an overview of the CyberWalk project. In Human Walking in Virtual Environments, pages 113-144. Springer. 
Gibson, J. J. (1933). Adaptation, After-Effect and Contrast in the Perception of Curved Lines. Journal of Experimental Psychology, 16(1):1.

Goertz, A., Makarski, M., Moldrzyk, C., and Weinzierl, S. (2007). Entwicklung eines achtkanaligen Lautsprechermoduls fur die Wellenfeldsynthese. FORTSCHRITTE DER AKUSTIK, 33(2):681.

Goldstein, E. B. (2010). Sensation and Perception.

Hodgson, E. and Bachmann, E. (2013). Comparing Four Approaches to Generalized Redirected Walking: Simulation and Live User Data. Visualization and Computer Graphics, IEEE Transactions on, 19(4):634-643.

Hodgson, E., Bachmann, E., and Thrash, T. (2014). Performance of Redirected Walking Algorithms in a Constrained Virtual World. Visualization and Computer Graphics, IEEE Transactions on, 20(4):579-587.

Hodgson, E., Bachmann, E., and Waller, D. (2011). Steering immersed users of virtual environments: Assessing the potential for spatial interference. ACM: Transactions on Applied Perception, 8:1-22.

Hollerbach, J. M., Xu, Y., Christensen, R., Jacobsen, S. C., et al. (2000). Design specifications for the second generation Sarcos Treadport locomotion interface. In Haptics Symposium, Proc. ASME Dynamic Systems and Control Division, volume 69, pages 1293-1298.

Huygens, C. and Blay, M. (1920). Traité de la lumiere, volume 13. Gauthier-Villars.

Insko, B., Meehan, M., Whitton, M., and Brooks, M. (2001). Passive Haptics Significantly Enhances Virtual Environments. In Proceedings of 4th Annual Presence Workshop.

Insko, B. E. (2001). Passive Haptics Significantly Enhances Virtual Environments. PhD thesis, Chapel Hill, NC, USA.

Kallie, C. S., Schrater, P. R., and Legge, G. E. (2007). Variability in stepping direction explains the veering behavior of blind walkers. Journal of Experimental Psychology: Human Perception and Performance, 33(1):183.

Kennedy, R. S., Lane, N. E., Berbaum, K. S., and Lilienthal, M. G. (1993). Simulator Sickness Questionnaire: An Enhanced Method for Quantifying Simulator Sickness. The International f. of Aviation Psychology, 3(3):203-220. 
Kohli, L., Burns, E., Miller, D., and Fuchs, H. (2005). Combining Passive Haptics with Redirected Walking. In Proceedings of the 2005 International Conference on Augmented Tele-existence, ICAT '05, pages 253-254, New York, NY, USA. ACM.

Kolasinski, E. M. (1995). Simulator Sickness in Virtual Environments. Technical report, DTIC Document.

Lackner, J. (1977). Induction of illusory self-rotation and nystagmus by a rotating sound-field. Aviation, space, and environmental medicine, 48(2):129-131.

Lee, S. J. and Hidler, J. (2008). Biomechanics of overground vs. treadmill walking in healthy individuals. Journal of Applied Physiology, 104(3):747-755.

Lindeman, R. W. (1999). Bimanual Interaction, Passive-Haptic Feedback, 3D Widget Representation, and Simulated Surface Constraints for Interaction in Immersive Virtual Environments. $\mathrm{PhD}$ thesis.

Lubos, P., Bruder, G., and Steinicke, F. (2014). Safe-\&-round: bringing redirected walking to small virtual reality laboratories. In Proceedings of the 2nd ACM symposium on Spatial user interaction, pages 154-154. ACM.

Meyer, F. (2015). Audio-visuelles Redirected Walking mit Oculus Rift und Wellenfeldsynthese. Unpublished Bachelor Thesis.

Mohler, B. J., Campos, J. L., Weyel, M. B., and Bülthoff, H. H. (2007). Gait Parameters while Walking in a Head-mounted Display Virtual Environment and the Real World . pages 85-88. motion sickness (2015). Dictionary.com Unabridged.

Neth, C., Souman, J., Engel, D., Kloos, U., Bülthoff, H., and Mohler, B. (2012). Velocity-Dependent Dynamic Curvature Gain for Redirected Walking. Visualization and Computer Graphics, IEEE Transactions on, 18(7):1041-1052.

Nilsson, N., Serafin, S., and Nordahl, R. (2014a). A comparison of four different approaches to reducing unintended positional drift during walking-In-Place locomotion. In Virtual Reality (VR), 2014 IEEE, pages 1-2.

Nilsson, N., Serafin, S., and Nordahl, R. (2014b). A comparison of different methods for reducing the unintended positional drift accompanying walking-in-place locomotion. In $3 D$ User Interfaces (3DUI), 2014 IEEE Symposium on, pages 103-110. 
Nitzsche, N., Hanebeck, U. D., and Schmidt, G. (2004). Motion Compression for Telepresent Walking in Large Target Environments. Presence: Teleoper. Virtual Environ., 13(1):44-60.

Nogalski, M. (2012). Gestengesteuerte Positionierung von Klangquellen einer Wellenfeldsynthese-Anlage mit Hilfe eines kamerabasierten 3D-Tracking-Systems. Bachelor thesis, Hamburg University for Applied Sciences.

Nogalski, M. and Fohl, W. (2015). Acoustically Guided Redirected Walking in a WFS System: Design of an Experiment to Identify Detection Thresholds. In Proceedings of the 12th Int. Conference on Sound and Music Computing (SMC-15), pages 419-426.

Peck, T. C., Fuchs, H., and Whitton, M. C. (2009). Evaluation of reorientation techniques and distractors for walking in large virtual environments. Visualization and Computer Graphics, IEEE Transactions on, 15(3):383-394.

proprioception (2015). Dictionary.com Unabridged.

Pulkki, V. (1997). Virtual Sound Source Positioning Using Vector Base Amplitude Panning. F. Audio Eng. Soc, 45(6):456-466.

Razzaque, S. (2005). Redirected Walking. PhD thesis, Chapel Hill, NC, USA. AAI3190299.

Razzaque, S., Kohn, Z., and Whitton, M. C. (2001). Redirected Walking. Technical report, Chapel Hill, NC, USA.

Razzaque, S., Swapp, D., Slater, M., Whitton, M. C., and Steed, A. (2002). Redirected walking in place. In ACM International Conference Proceeding Series, volume 23, pages 123-130.

Ruddle, R. A. and Lessels, S. (2009). The Benefits of Using a Walking Interface to Navigate Virtual Environments. ACM Trans. Comput.-Hum. Interact., 16(1):5:1-5:18.

Sekiya, N., Nagasaki, H., Ito, H., and Ruruna, T. (1996). The invariant relationship between step length and step rate during free walking. J Hum Mov Stud, 30:241-57.

Serafin, S., Nilsson, N., Sikstrom, E., De Goetzen, A., and Nordahl, R. (2013). Estimation of Detection Thresholds for Acoustic Based Redirected Walking Techniques. In Virtual Reality (VR), 2013 IEEE, pages 161-162.

Sinitski, E. H., Lemaire, E. D., Baddour, N., Besemann, M., Dudek, N. L., and Hebert, J. S. (2015). Fixed and self-paced treadmill walking for able-bodied and transtibial amputees in a multi-terrain virtual environment. Gait \& posture, 41(2):568-573. 
Souman, J. L., Frissen, I., Sreenivasa, M. N., and Ernst, M. O. (2009). Walking straight into circles. Current Biology, 19(18):1538-1542.

Souman, J. L., Giordano, P. R., Frissen, I., Luca, A. D., and Ernst, M. O. (2010). Making Virtual Walking Real: Perceptual Evaluation of a New Treadmill Control Algorithm. ACM Trans. Appl. Percept., 7(2):11:1-11:14.

Souman, J. L., Giordano, P. R., Schwaiger, M., Frissen, I., Thümmel, T., Ulbrich, H., Luca, A. D., Bülthoff, H. H., and Ernst, M. O. (2011). Cyberwalk: Enabling unconstrained omnidirectional walking through virtual environments. ACM Transactions on Applied Perception (TAP), $8(4): 25$.

Sreenivasa, M., Frissen, I., Souman, J., and Ernst, M. (2008). Walking along curved paths of different angles: the relationship between head and trunk turning. Experimental Brain Research, 191(3):313-320.

Stanney, K. M., Kennedy, R. S., and Drexler, J. M. (1997). Cybersickness is Not Simulator Sickness. Proceedings of the Human Factors and Ergonomics Society Annual Meeting, 41(2):1138-1142.

Steinicke, F., Bruder, G., Hinrichs, K., Jerald, J., Frenz, H., and Lappe, M. (2009). Real Walking through Virtual Environments by Redirection Techniques. FVRB - Journal of Virtual Reality and Broadcasting, 6(2009)(2).

Steinicke, F., Bruder, G., Jerald, J., Frenz, H., and Lappe, M. (2008a). Analyses of Human Sensitivity to Redirected Walking. In Proceedings of the 2008 ACM Symposium on Virtual Reality Software and Technology, VRST '08, pages 149-156, New York, NY, USA. ACM.

Steinicke, F., Bruder, G., Jerald, J., Frenz, H., and Lappe, M. (2010). Estimation of Detection Thresholds for Redirected Walking Techniques. Visualization and Computer Graphics, IEEE Transactions on, 16(1):17-27.

Steinicke, F., Bruder, G., Kohli, L., Jerald, J., and Hinrichs, K. (2008b). Taxonomy and Implementation of Redirection Techniques for Ubiquitous Passive Haptic Feedback. In Cyberworlds, 2008 International Conference on, pages 217-223.

Steinicke, F., Bruder, G., Ropinski, T., and Hinrichs, K. H. (2008c). Moving Towards Generally Applicable Redirected Walking. In Proceedings of the Virtual Reality International Conference (VRIC), pages 15-24. IEEE Press. 
Steinicke, F., Bruder, G., Ropinski, T., Hinrichs, K. H., Frenz, H., and Lappe, M. (2008d). Generic Redirected Walking \& Dynamic Passive Haptics: Evaluation and Implications for Virtual Locomotion Interfaces. In Proceedings of IEEE Symposium on 3D User Interfaces (3DUI) (Poster Presentation), pages 147-148. IEEE Press.

Steinicke, F., Ropinski, T., Bruder, G., and Hinrichs, K. (2008e). The Holodeck Construction Manual.

Steinicke, F., Ropinski, T., Bruder, G., Hinrichs, K., Frenz, H., and Lappe, M. (2008f). A Universal Virtual Locomotion System: Supporting Generic Redirected Walking and Dynamic Passive Haptics within Legacy 3D Graphics Applications. In Virtual Reality Conference, 2008. VR '08. IEEE, pages 291-292.

Steinicke, F., Welzel, H., Bruder, G., and Hinrichs, K. (2008g). A User Guidance Approach for Passive Haptic Environments. In 14th Eurographics symposium on virtual environments (EGVE 2008), pages 31-34.

Stevenson, A. (2010). Oxford Dictionary of English. Oxford reference online premium. OUP Oxford.

Streuber, S., de la Rosa, S., Trutoiu, L., Bülthoff, H. H., and Mohler, B. J. (2009). Does Brief Exposure to a Self-avatar Effect Common Human Behaviors in Immersive Virtual Environments? In Eurographics 2009-Short Papers, pages 33-36. The Eurographics Association.

Su, J. (2007). Motion compression for telepresence locomotion. Presence: Teleoperators and Virtual Environments, 16(4):385-398.

synthetic wave audio products GmbH (2015). WFS principle. http://WwW . syntheticwave.de/Wavefieldsynthes is .htm. Online: 11.08.2015.

Tachi, S., Maeda, T., Hirata, R., and Hoshino, H. (1994). A Construction Method of Virtual Haptic Space. In Proceedings of the 4th International Conference on Artificial Reality and Tele-Existence (ICAT'94).

The Center For New Music and Audio Technology (CNMAT) (2015). Open Sound Control.

Tzanetakis, G., Martins, L. G., McNally, K., and Jones, R. (2010). Stereo Panning Information for Music Information Retrieval Tasks. F. Audio Eng. Soc, 58(5):409-417.

Usoh, M., Arthur, K., Whitton, M. C., Bastos, R., Steed, A., Slater, M., and Brooks, Jr., F. P. (1999). Walking $>$ Walking-in-place $>$ Flying, in Virtual Environments. In Proceedings of the 26th 
Annual Conference on Computer Graphics and Interactive Techniques, SIGGRAPH '99, pages 359-364, New York, NY, USA. ACM Press/Addison-Wesley Publishing Co.

Vu, D.-S., Foucault, S., Gosselin, C., and Kovecses, J. (2015). Design of a locomotion interface for gait simulation based on belt-driven parallel mechanisms. In Robotics and Automation (ICRA), 2015 IEEE International Conference on, pages 1581-1586. IEEE.

Walker, J. (2013). Redirected Walking in Virtual Environments. http://cs.mtu.edu/ jwwalker/files/cs5760-jwwalker-topicpaper.pdf.

Wallach, H. (1987). Perceiving a stable environment when one moves. Annual review of psychology, 38(1):1-29.

Wertheim, A. H. (1994). Motion perception during selfmotion: The direct versus inferential controversy revisited. Behavioral and Brain Sciences, 17(02):293-311.

Wikipedia (2014). Star Treck Technolog. http://en.wikipedia.org/wiki/ Holodeck\#Holodeck. Online: 15.08.2014.

Williams, B., Narasimham, G., McNamara, T. P., Carr, T. H., Rieser, J. J., and Bodenheimer, B. (2006a). Updating Orientation in Large Virtual Environments Using Scaled Translational Gain. In Proceedings of the 3rd Symposium on Applied Perception in Graphics and Visualization, APGV '06, pages 21-28, New York, NY, USA. ACM.

Williams, B., Narasimham, G., Rump, B., McNamara, T. P., Carr, T. H., Rieser, J., and Bodenheimer, B. (2006b). Exploring large virtual environments with an hmd on foot. In ACM SIGGRAPH 2006 Research posters, page 182. ACM.

Williams, B., Narasimham, G., Rump, B., McNamara, T. P., Carr, T. H., Rieser, J., and Bodenheimer, B. (2007). Exploring Large Virtual Environments with an HMD when Physical Space is Limited. In Proceedings of the 4th Symposium on Applied Perception in Graphics and Visualization, APGV '07, pages 41-48, New York, NY, USA. ACM.

Zahorik, P., Brungart, D. S., and Bronkhorst, A. W. (2005). Auditory distance perception in humans: A summary of past and present research. Acta Acustica united with Acustica, 91(3):409-420. 
Hiermit versichere ich, dass ich die vorliegende Arbeit ohne fremde Hilfe selbständig verfasst und nur die angegebenen Hilfsmittel benutzt habe.

Hamburg, 27th August 2015 Malte Nogalski 